\title{
Diastereoselective Additions of Allylmagnesium Reagents to $\alpha$-Substituted Ketones When Stereochemical Models Cannot Be Used
}

\author{
Nicole D. Bartolo, ${ }^{1}$ Krystyna M. Demkiw, ${ }^{1}$ Elizabeth M. Valentín, ${ }^{2}$ Chunhua T. Hu, ${ }^{1}$ Alya A. \\ Arabi, ${ }^{3}$ and K. A. Woerpel ${ }^{1 *}$ \\ ${ }^{1}$ Department of Chemistry, New York University, 100 Washington Square East, New York, NY \\ 10003 USA \\ ${ }^{2}$ St. Mary's College of California, 1928 St. Marys Road, Moraga, CA 94575 USA \\ ${ }^{3}$ Biochemistry Department, College of Medicine and Health Sciences, United Arab Emirates \\ University, PO Box 17666, Al Ain, UAE and Centre for Computational Science, University \\ College London, 20 Gordon St, London, WC1H 0AJ, United Kingdom
}

\section{Supporting Information}

\section{Table of Contents}

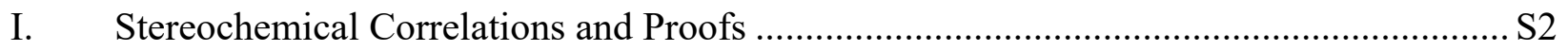

A. Assignment of Relative Stereochemical Configuration by ${ }^{1} \mathrm{H}$ NMR Coupling

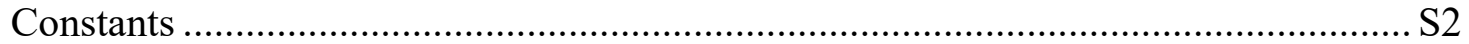

B. Assignment of the Relative Stereochemical Configuration by Epoxide Formation .... S5

II. Computational Investigations ...................................................................................

A. Transition State Calculations................................................................................. S7

B. Cartesian Coordinates (in $\AA$ ) for Complex of AllylMg(Cl)(THF) and Ketone $5 \mathrm{~b}$ Using the Polarizable Continuum Solvent Model with Dielectric Constant $=7.43$

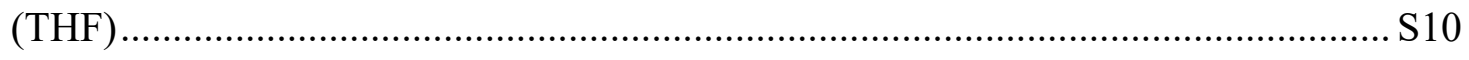

C. Calculation of Ground-State Conformers of Ketone 30 ............................................ S20

D. Calculation of Ground-State Conformers of Ketone 37............................................ S22

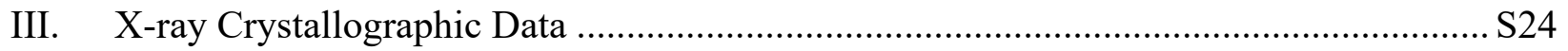

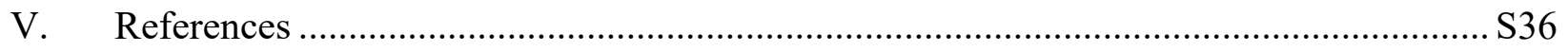

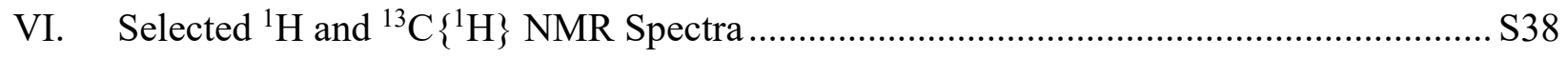




\section{Stereochemical Correlations and Proofs}

\section{A. Assignment of Relative Stereochemical Configuration by ${ }^{1}$ H NMR Coupling Constants}

Peaks were assigned in the ${ }^{1} \mathrm{H}$ NMR spectra by ${ }^{1} \mathrm{H}$ NMR chemical shifts, ${ }^{1} \mathrm{H}$ NMR coupling constants, and HSQC. The stereochemical configurations of the products were determined using ${ }^{1} \mathrm{H}$ NMR coupling constants because the coupling constants between protons on six-membered rings are well-documented. ${ }^{1}$ Protons will either adopt an axial or equatorial orientation on the ring, and the coupling between adjacent protons (the coupling constant, $J$ ) will help to elucidate the structure of the compound. The relative positioning between protons on the six-membered ring are as follows: axial-axial, 8 to $13 \mathrm{~Hz}$; axial-equatorial, 2 to $6 \mathrm{~Hz}$; equatorial-equatorial, 2 to $9 \mathrm{~Hz}{ }^{1}$ A-values of substituents on six-membered rings provide insight on the positioning of the $\mathrm{OH}$ group on the ring, which is expected to be axial in allylated products. ${ }^{2,3}$

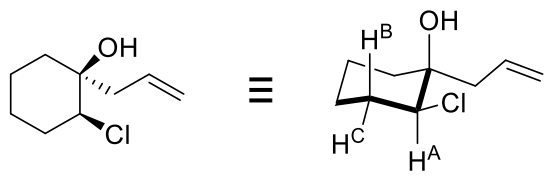

Alcohol anti-7a (1,2-trans, major diastereomer):

${ }^{1} \mathrm{H}$ NMR $\left(400 \mathrm{MHz}, \mathrm{CDCl}_{3}\right) \delta 3.99\left(\mathrm{H}^{\mathrm{A}}: \mathrm{dd}, J=10.3 \mathrm{~Hz}\left(\mathrm{ax}^{\mathrm{A}}-\mathrm{ax}^{\mathrm{B}}\right), 5.4 \mathrm{~Hz}\left(\mathrm{ax}^{\mathrm{A}}-\mathrm{eq}^{\mathrm{C}}\right)\right)$

Note: The splitting pattern of $\mathrm{H}^{\mathrm{A}}$ indicates that $\mathrm{H}^{\mathrm{A}}$ is axial $(\mathrm{dd}, J=10.3,5.4 \mathrm{~Hz})$; the structure is 1,2-trans diequatorial because the larger allyl group should adopt an equatorial orientation, as noted in X-ray crystallographic structures of similar compounds. ${ }^{4-6}$

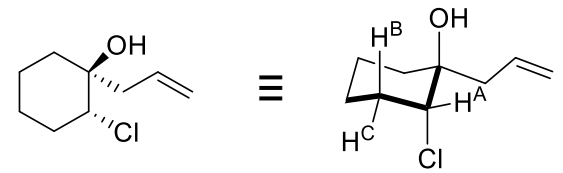

Alcohol syn-7a (1,2-cis, minor diastereomer):

${ }^{1} \mathrm{H}$ NMR $\left(400 \mathrm{MHz}, \mathrm{CDCl}_{3}\right) \delta 3.97\left(\mathrm{H}^{\mathrm{A}}: \mathrm{dd}, J=7.5 \mathrm{~Hz}\left(\mathrm{eq}^{\mathrm{A}}-\mathrm{eq}^{\mathrm{C}}\right), 3.7 \mathrm{~Hz}\left(\mathrm{eq}^{\mathrm{A}}-\mathrm{ax}^{\mathrm{B}}\right)\right)$

Note: The splitting pattern of $\mathrm{H}^{\mathrm{A}}$ indicates that $\mathrm{H}^{\mathrm{A}}$ is equatorial $(\mathrm{dd}, J=7.5,3.7 \mathrm{~Hz})$; the structure is 1,2 -cis. 


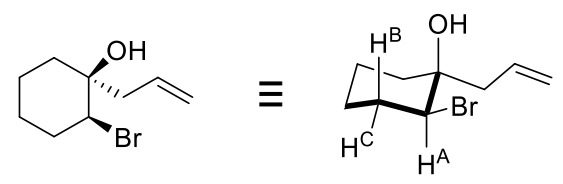

Alcohol anti-7b (1,2-trans, major diastereomer):

${ }^{1} \mathrm{H}$ NMR $\left(400 \mathrm{MHz}, \mathrm{CDCl}_{3}\right) \delta 4.20\left(\mathrm{H}^{\mathrm{A}}: \mathrm{dd}, J=11.3 \mathrm{~Hz}\left(\mathrm{ax}^{\mathrm{A}}-\mathrm{ax}^{\mathrm{B}}\right), 4.5 \mathrm{~Hz}\left(\mathrm{ax}^{\mathrm{A}}-\mathrm{eq}^{\mathrm{C}}\right)\right)$

Note: The splitting pattern of $\mathrm{H}^{\mathrm{A}}$ indicates that $\mathrm{H}^{\mathrm{A}}$ is axial $(\mathrm{dd}, J=11.3,4.5 \mathrm{~Hz})$; the structure is 1,2-trans diequatorial because the larger allyl group should adopt an equatorial orientation, as noted in X-ray crystallographic structures of similar compounds. ${ }^{4-6}$

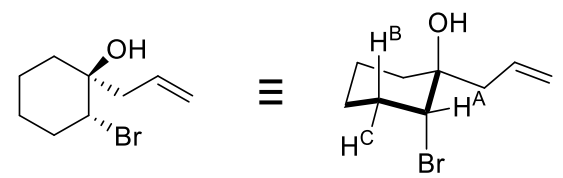

Alcohol syn-7b (1,2-cis, minor diastereomer):

${ }^{1} \mathrm{H}$ NMR $\left(400 \mathrm{MHz}, \mathrm{CDCl}_{3}\right) \delta 4.17\left(\mathrm{H}^{\mathrm{A}}: \mathrm{dd}, J=8.6 \mathrm{~Hz}\left(\mathrm{eq}^{\mathrm{A}}-\mathrm{eq}^{\mathrm{C}}\right), 3.9 \mathrm{~Hz}\left(\mathrm{eq}^{\mathrm{A}}-\mathrm{ax}^{\mathrm{B}}\right)\right)$

Note: The splitting pattern of $\mathrm{H}^{\mathrm{A}}$ indicates that $\mathrm{H}^{\mathrm{A}}$ is equatorial $(\mathrm{dd}, J=8.6,3.9 \mathrm{~Hz})$; the structure is 1,2 -cis.

The relative stereochemistry of alcohol anti-10a was assigned by comparison of the spectroscopic data to that of alcohol anti-10b, whose structure was determined by X-ray crystallographic analysis. The similar coupling constants $(J)$ of alcohol anti-10a and alcohol anti-10b indicate that the relative stereochemical configuration of the major diastereomer anti-10a positions the chlorine atom and allyl group in a trans relationship. Consequently, the similar coupling constants $(J)$ of alcohol syn-10a and alcohol syn-10b indicate that the relative stereochemical configuration of the minor diastereomer syn-10a positions the chlorine atom and allyl group in a cis relationship.

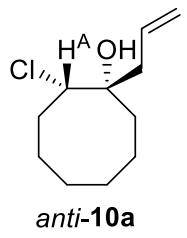

Alcohol anti-10a (1,2-trans, major diastereomer):

${ }^{1} \mathrm{H}$ NMR (400 MHz, $\mathrm{CDCl}_{3}$, diagnostic peaks) $\delta 4.25\left(\mathrm{H}^{\mathrm{A}}: \mathrm{dd}, J=8.9,0.9 \mathrm{~Hz}\right)$ 


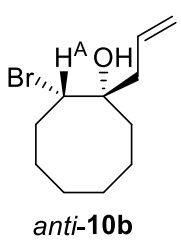

Alcohol anti-10b (1,2-trans, major diastereomer):

${ }^{1} \mathrm{H} \mathrm{NMR}\left(400 \mathrm{MHz}, \mathrm{CDCl}_{3}\right.$, diagnostic peaks) $\delta 4.46\left(\mathrm{H}^{\mathrm{A}}: \mathrm{dd}, J=8.9,1.0 \mathrm{~Hz}\right)$

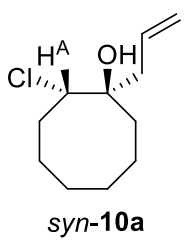

Alcohol syn-10a (1,2-cis, minor diastereomer):

${ }^{1} \mathrm{H} \mathrm{NMR}\left(400 \mathrm{MHz}, \mathrm{CDCl}_{3}\right.$, diagnostic peaks) $\delta 4.81\left(\mathrm{H}^{\mathrm{A}}: \mathrm{dd}, J=10.6,4.1 \mathrm{~Hz}\right)$

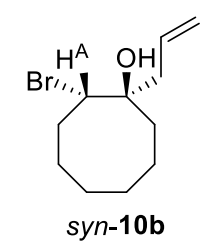

Alcohol syn-10b (1,2-cis, minor diastereomer):

${ }^{1} \mathrm{H}$ NMR (400 MHz, $\mathrm{CDCl}_{3}$, diagnostic peaks) $\delta 4.56\left(\mathrm{H}^{\mathrm{A}}: \mathrm{dd}, J=9.9,4.5 \mathrm{~Hz}\right)$ 


\section{B. Assignment of the Relative Stereochemical Configuration by Epoxide Formation}

The stereochemical configurations of the alcohols anti-6a, anti-6b, anti-8a, anti-8b, anti-9a, and anti-9b were assigned by derivatization. An epoxide forms as a result of an intramolecular displacement of the halogen atom by the hydroxyl group upon treatment with a base. An antiorientation between the alcohol and the halogen atom is necessary for this transformation to occur. ${ }^{7}$ This orientation is only found in the minor diastereomer of each addition reaction.

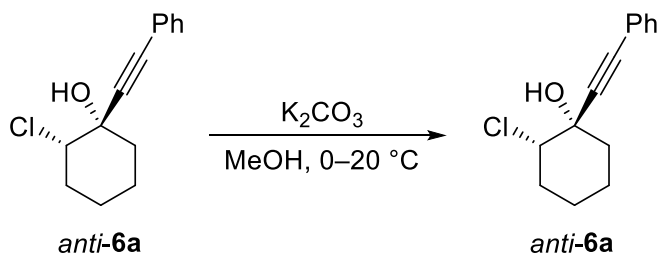

Note: The absence of the formation of epoxide indicated that the relative stereochemical configuration of major diastereomer anti-6a positions the chlorine atom and the phenylacetylene group in a trans relationship.

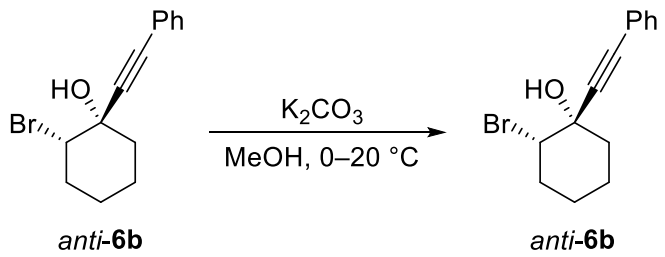

Note: The absence of the formation of epoxide indicated that the relative stereochemical configuration of major diastereomer anti-6b positions the bromine atom and the phenylacetylene group in a trans relationship.

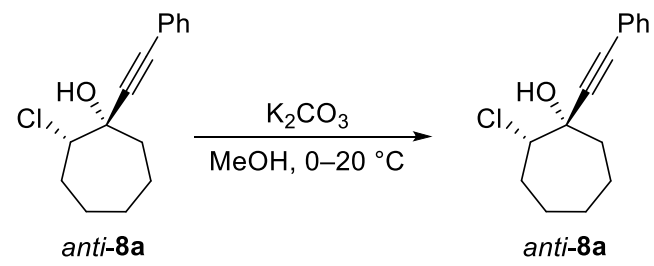

Note: The absence of the formation of epoxide indicated that the relative stereochemical configuration of major diastereomer anti-8a positions the chlorine atom and the phenylacetylene group in a trans relationship. 


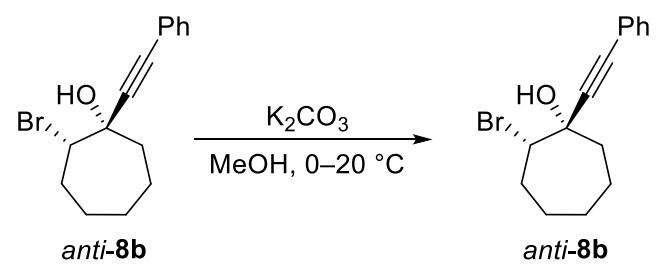

Note: The absence of the formation of epoxide indicated that the relative stereochemical configuration of major diastereomer anti-8b positions the bromine atom and the phenylacetylene group in a trans relationship.

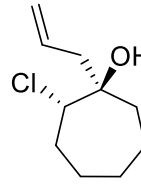

syn-9a

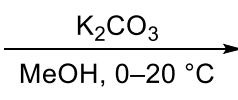

Note: Based on the formation of epoxide 12, the relative stereochemical configuration of minor diastereomer syn-9a positions the chlorine atom and the allyl group in a cis relationship. Consequently, the relative stereochemical configuration of the major diastereomer anti-9a positions the chlorine atom and the allyl group in a trans relationship.

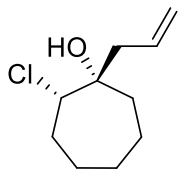

anti-9a

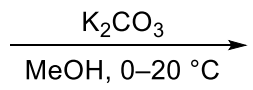

$\mathrm{MeOH}, 0-20^{\circ} \mathrm{C}$

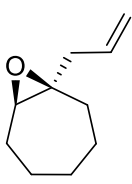

12

Note: The absence of the formation of epoxide $\mathbf{1 2}$ indicated that the relative stereochemical configuration of major diastereomer anti-9a positions the chlorine atom and the allyl group in a trans relationship.

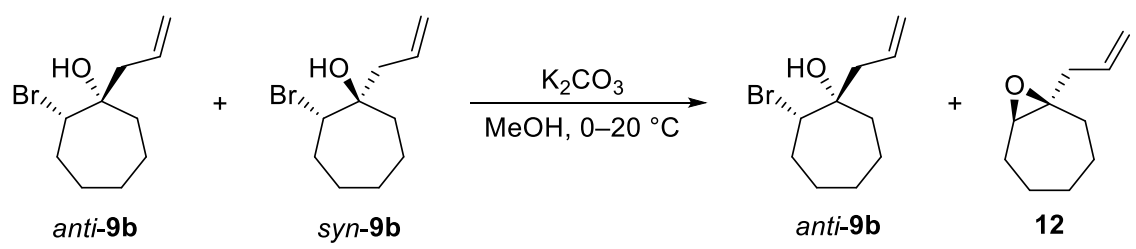

Note: The absence of minor diastereomer syn-9b in the unpurified reaction mixture and the formation of epoxide $\mathbf{1 2}$ indicated that the relative stereochemical configuration of minor diastereomer syn-9b positions the bromine atom and the allyl group in a cis relationship. Based on the recovery of the major diastereomer anti-9b and the relative stereochemical configuration of minor diastereomer $\operatorname{syn} \mathbf{- 9 \mathbf { b }}$, the relative stereochemical configuration of alcohol anti-9b is a trans relationship between the bromine atom and the allyl group. 


\section{Computational Investigations}

\section{A. Transition State Calculations}

The conformational preferences of 2-bromocyclooctanone were first determined to compare to those of the published computational studies ${ }^{8}$ using the Spartan $^{9}$ program. A conformational search (MMFF) of 1000 potential conformers produced nine low-energy conformers. These conformers were then minimized (B3LYP/aug-cc-pVDZ), which produced three conformers equivalent to the 2-bromocyclooctanone conformers I-III in the published work. ${ }^{8}$ The values determined were within $0.3 \mathrm{kcal} / \mathrm{mol}$ of those reported.
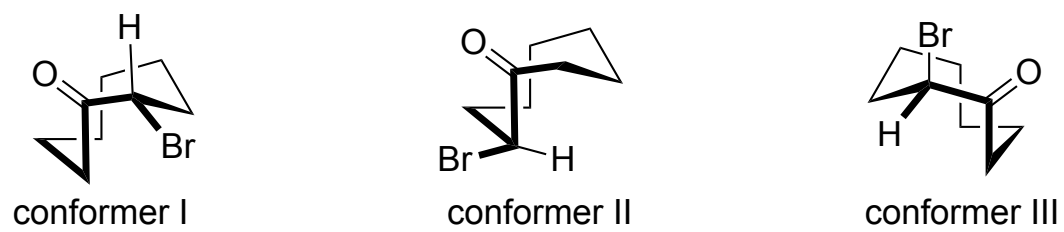

Transition states were initially identified from conformers I-III using B3LYP/6-31G* by first complexation to a $\mathrm{Mg}(\mathrm{Cl})(\mathrm{THF})$ fragment then searching for a transition state. This magnesiumcontaining fragment was chosen because it led to one of the low-energy transition structures for addition of methylmagnesium reagents to aldehydes ${ }^{10}$ and because it would have fewer heavy atoms and fewer parameters that would require optimization. A number of structures would still be possible, however, even with this small metal fragment. The magnesium atom could be connected to either of two sides of the carbonyl group, and two possible stereochemical configurations at the magnesium atom would be possible. The calculations were initiated with the magnesium atom connected to the oxygen atom on the face away from the substituent, and the THF ligand was placed in an orientation that was sterically least congested, as shown in S1 below. This assumption did not appear to be critical to the energy of a ketone-Grignard complex: the energies of a complex of conformer $\mathrm{I}^{8}$ and the magnesium fragment with different orientations at the oxygen atom and at the magnesium atom were similar (within $\sim 1 \mathrm{kcal} / \mathrm{mol}$ ) using B3LYP/6$31 \mathrm{G}^{*}$ or using a functional that contains a dispersion correction ${ }^{11}(\omega \mathrm{B} 97 \mathrm{X}-\mathrm{D}$ with the cc-pVDZ basis set). Calculations were continued from the lowest energy complexes in each conformer, which reduced the numbers of structures that needed to be considered. Although a triple zeta basis should give better accuracy, the cc-pVDZ basis set was used to accelerate the calculations, which involved 46 atoms, 431 basis functions, and 196 electrons even at this level.

Transition states were identified by a two-step process. The first step involved identifying a starting point for locating a transition state. This step was achieved by determining the energy of the complex as a function of the distance between the terminus of the allyl group and the carbonyl carbon atom. The low-energy complexes for each conformer were subjected to an energy profile calculation (B3LYP/6-31G*) with the appropriate $\mathrm{C}-\mathrm{C}$ bond lengths reduced by $0.1-0.3 \AA$ increments from $\sim 4-6 \AA$ to $\sim 2.0 \AA$. The energies of the complexes were generally unchanged until the distance reached $\sim 2.5 \AA$, at which point the energy decreased significantly, indicating bond 
formation. In most cases, a second profile with smaller differences in $\mathrm{C}-\mathrm{C}$ bond lengths $(<0.1 \AA)$ was used to identify a reasonable starting point just before the energy sharply decreased as a function of distance. In a second step, those starting geometries were subjected to a transition state search (B3LYP/6-31G*). In the case of a complex from conformer I (i.e., S1), transition state energies differed by $<1 \mathrm{kcal} / \mathrm{mol}$ regardless of specific starting point, including geometry at the magnesium atom and the face to which the magnesium atom was complexed.

Additional optimization of transition state geometries was pursued for conformers I-III. Two modes of addition were considered for conformer I, as discussed in the text (the discussion accompanying Scheme 5). Transition states were identified using B3LYP/6-31G* and other methods, as noted in Table S1, which, in some cases, required selecting a different starting geometry (i.e., carbon-carbon bond distance) from the profiles noted above using B3LYP/6-31G*. As illustrated in Table S1, relative energies were largely independent of the functional and basis set, and the relative ordering of transition state energies did not depend upon whether the calculations were conducted in the gas phase or using a polarized continuum model to model the effect of the solvent, THF. ${ }^{12}$ In all cases, a larger-magnitude $(\sim 200 i-300 i)$ imaginary vibration corresponding to carbon-carbon bond formation were identified. In some cases, other lowfrequency imaginary vibrations were found (approximately $50 i \mathrm{~cm}^{-1}$ or lower) that, by visual inspection, corresponded to rotation of the THF group or bending at the magnesium atom. Tightening the tolerance of the optimization (gradient tolerance $=0.0002$ ) led, for most calculations, to transition structures with only one vibration. This additional optimization changed the energy of the structure by $<0.1 \mathrm{kcal} / \mathrm{mol}$.

In the case of the $\omega \mathrm{B} 97 \mathrm{X}-\mathrm{D} / \mathrm{cc}-\mathrm{pVDZ} / \mathrm{THF}$ calculations, it was not possible to avoid all lowfrequency vibrations. Tight restrictions on tolerances of geometry and gradient ("GEOMTOL=TIGHT") did not remove the low-frequency vibrations noted above nor did the energies change by more than $0.1 \mathrm{kcal} / \mathrm{mol}$, but these calculations were time-intensive. Generally, low-frequency imaginary vibrations do not alter energies significantly. ${ }^{13,14}$

These transition structures, regardless of the method, are consistent with the experimental results. As discussed in the text accompanying Scheme 5, the major product could be formed by reaction through transition structures 22', 23', or 24'. Transition structures 22' and 23', however, would be relatively high energy, so reaction through these transition structures would be slower than exchange of the ketone ligand. Therefore, reaction through 22' and 23' would not be consistent with the competition experiments. Reaction through transition state 24', however, would form the major product and would be consistent with the competition experiments. Reaction through transition structure 21', which is higher in energy than 22' by $\sim 5 \mathrm{kcal} / \mathrm{mol}$, would give the minor stereoisomer of product, and the higher energy is consistent with the fact that this product is not formed in high quantities. 
Table S1. Relative free energies $\left(\Delta \mathrm{G}^{*}\right)$ of transition states $(\mathrm{kcal} / \mathrm{mol})$ compared to the complex as a function of method (structures summarized in Scheme 5 of the text).

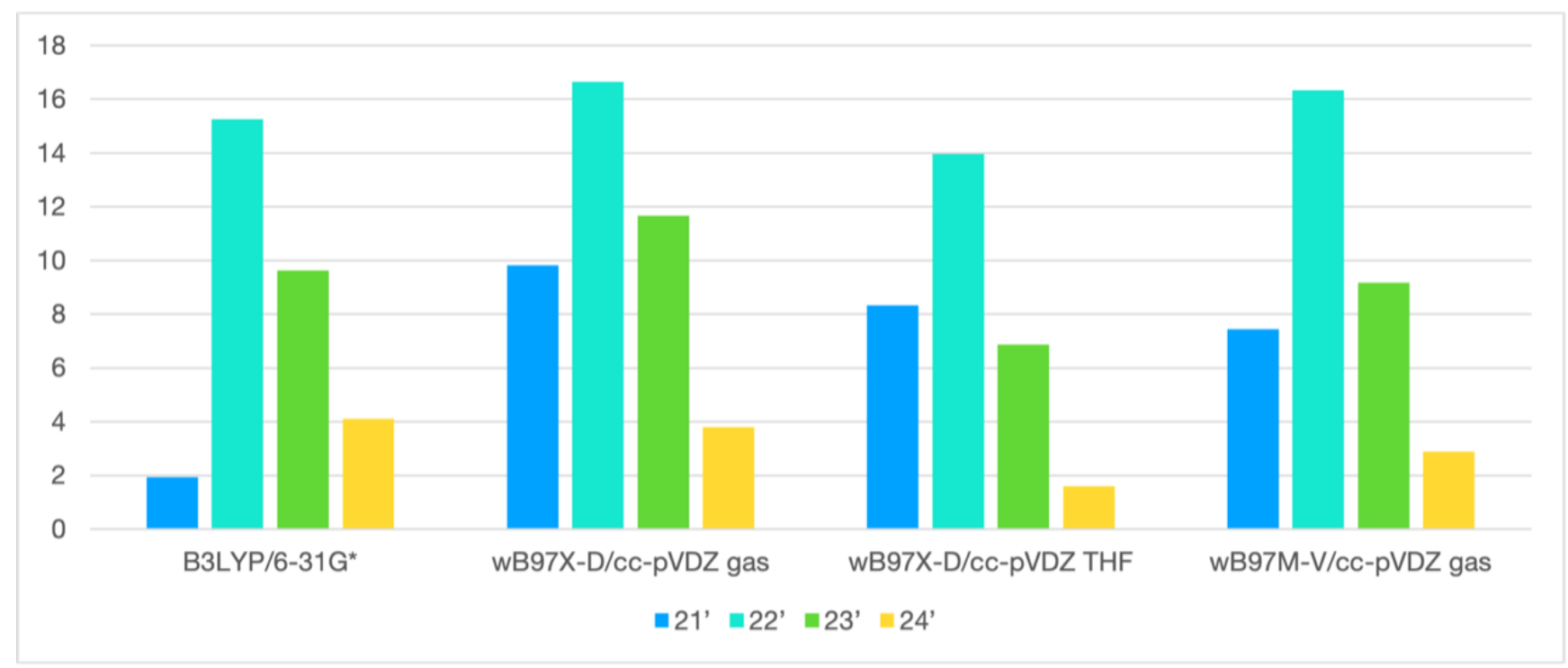




\section{B. Cartesian Coordinates (in Å) for Complex of AllylMg(Cl)(THF) and Ketone 5b Using the Polarizable Continuum Solvent Model with Dielectric Constant $=7.43$ (THF)}

Ground state complex (S1):

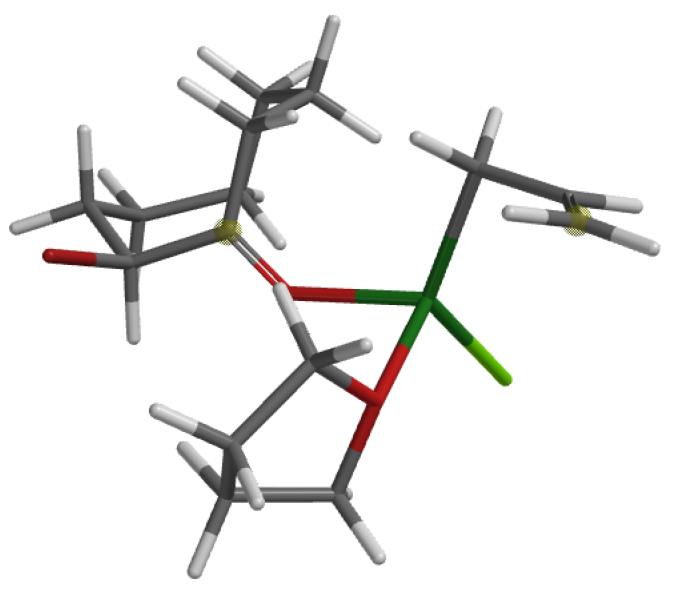

SCF Total Energy: -3972.1256253 Hartrees

Four imaginary frequencies: $66 i \mathrm{~cm}^{-1}, 42 i \mathrm{~cm}^{-1}, 20 i \mathrm{~cm}^{-1}, 11 i \mathrm{~cm}^{-1}$, corresponding to geometry changes at $\mathrm{Mg}$ and rotation of the allyl group $\mathrm{C}-\mathrm{C}$ bond length: $6.171 \AA$

\begin{tabular}{cccc} 
Atom & $\mathrm{X}$ & $\mathrm{Y}$ & $\mathrm{Z}$ \\
\hline $\mathrm{C}$ & -2.6623283 & -0.8108513 & 0.8274879 \\
$\mathrm{C}$ & -0.3424210 & 0.1229018 & 1.1167604 \\
$\mathrm{C}$ & 0.2442226 & 0.5005579 & 2.4606126 \\
$\mathrm{C}$ & -0.6634779 & 0.2879370 & 3.6613842 \\
$\mathrm{C}$ & -0.9272265 & -1.1945799 & 3.9553582 \\
$\mathrm{C}$ & -1.5599835 & -2.0286145 & 2.8316983 \\
$\mathrm{C}$ & -2.8463667 & -1.4515307 & 2.2061715 \\
$\mathrm{C}$ & -1.7697751 & 0.4470393 & 0.7899556 \\
$\mathrm{Br}$ & 0.6736697 & 2.4150820 & 2.2313322 \\
$\mathrm{H}$ & -3.6431306 & -0.5158045 & 0.4260154 \\
$\mathrm{H}$ & -2.2492614 & -1.5570404 & 0.1268365 \\
$\mathrm{H}$ & 1.2146295 & 0.0018422 & 2.5733994 \\
$\mathrm{H}$ & -1.6104315 & 0.8293092 & 3.5144431 \\
$\mathrm{H}$ & -0.1774620 & 0.7431972 & 4.5362410 \\
$\mathrm{H}$ & -1.5871133 & -1.2282757 & 4.8377073 \\
$\mathrm{H}$ & 0.0190319 & -1.6727321 & 4.2575364 \\
$\mathrm{H}$ & -1.7772877 & -3.0197466 & 3.2578552 \\
$\mathrm{H}$ & -0.8183409 & -2.2263653 & 2.0377451
\end{tabular}




\begin{tabular}{|c|c|c|c|}
\hline $\mathrm{H}$ & -3.3089426 & -0.7223677 & 2.8942939 \\
\hline $\mathrm{H}$ & -3.5834324 & -2.2601771 & 2.0875681 \\
\hline $\mathrm{H}$ & -2.1618336 & 1.2151647 & 1.4696033 \\
\hline $\mathrm{H}$ & -1.7759804 & 0.8518301 & -0.2328918 \\
\hline $\mathrm{O}$ & 0.3843279 & -0.4599140 & 0.3133922 \\
\hline $\mathrm{Mg}$ & 0.2847026 & -0.9874404 & -1.6966805 \\
\hline $\mathrm{O}$ & 1.9864501 & 0.1445284 & -1.9627078 \\
\hline $\mathrm{Cl}$ & 0.8669915 & -3.2429210 & -1.6655117 \\
\hline $\mathrm{C}$ & -1.3115065 & -0.0798631 & -2.8024073 \\
\hline $\mathrm{H}$ & -1.4067637 & 0.9793452 & -2.4984712 \\
\hline $\mathrm{H}$ & -2.2515077 & -0.6093008 & -2.5670885 \\
\hline $\mathrm{C}$ & -0.9495106 & -0.2104299 & -4.2250341 \\
\hline $\mathrm{H}$ & -1.1243583 & -1.2052950 & -4.6634310 \\
\hline $\mathrm{C}$ & -0.3718748 & 0.7074016 & -5.0268578 \\
\hline $\mathrm{H}$ & -0.1718177 & 1.7259034 & -4.6740809 \\
\hline $\mathrm{H}$ & -0.0861086 & 0.4675913 & -6.0541425 \\
\hline $\mathrm{C}$ & 3.2216028 & -0.2178317 & -1.2937516 \\
\hline $\mathrm{H}$ & 3.0488270 & -1.1603240 & -0.7579490 \\
\hline $\mathrm{H}$ & 3.9956027 & -0.3725308 & -2.0615602 \\
\hline $\mathrm{C}$ & 1.8107278 & 1.5887040 & -1.9281492 \\
\hline $\mathrm{H}$ & 0.9103819 & 1.8039993 & -1.3305264 \\
\hline $\mathrm{H}$ & 1.6513783 & 1.9348157 & -2.9574095 \\
\hline $\mathrm{C}$ & 3.0712974 & 2.1397306 & -1.2718214 \\
\hline $\mathrm{H}$ & 2.8617770 & 3.0513737 & -0.6970825 \\
\hline $\mathrm{H}$ & 3.8310659 & 2.3733660 & -2.0326295 \\
\hline $\mathrm{C}$ & 3.5326414 & 0.9687627 & -0.4012509 \\
\hline $\mathrm{H}$ & 2.9331384 & 0.9148689 & 0.5206751 \\
\hline $\mathrm{H}$ & 4.5957771 & 1.0186842 & -0.1326376 \\
\hline
\end{tabular}

\section{At $298 \mathrm{~K}$}

Zero Point Energy: $1026.83 \mathrm{~kJ} / \mathrm{mol}$ (ZPE)

Temperature Correction: $53.42 \mathrm{~kJ} / \mathrm{mol}$ (vibration + gas law + rotation + translation)

Enthalpy Correction: $1080.25 \mathrm{~kJ} / \mathrm{mol}$ (ZPE + temperature correction)

Enthalpy: -3971.714180 au (Electronic Energy + Enthalpy Correction)

Entropy: $637.13 \mathrm{~J} / \mathrm{mol} \cdot \mathrm{K}$

Gibbs Energy: -3971.786532 au (Enthalpy - T*Entropy)

$\mathrm{Cv}: 366.70 \mathrm{~J} / \mathrm{mol} \cdot \mathrm{K}$ 
TS from lowest energy conformer (21'):

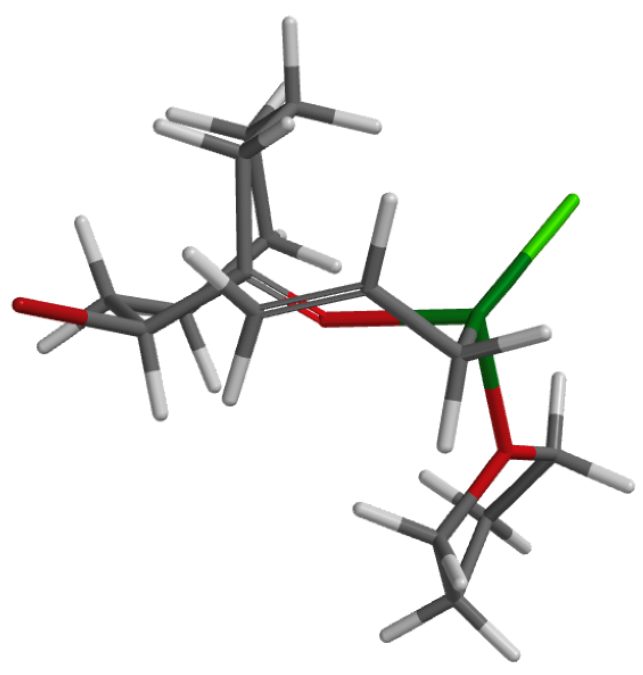

SCF Total Energy: -3972.112588 Hartrees

Three imaginary frequencies: $276 i \mathrm{~cm}^{-1}$, corresponding to $\mathrm{C}-\mathrm{C}$ bond formation; $53 i \mathrm{~cm}^{-1}$, corresponding to bending at $\mathrm{Mg} ; 9 i \mathrm{~cm}^{-1}$, corresponding to rotation about $\mathrm{O}-\mathrm{Mg}$ $\mathrm{C}-\mathrm{C}$ bond length: $2.457 \AA$

\begin{tabular}{cccc} 
Atom & $\mathrm{X}$ & $\mathrm{Y}$ & $\mathrm{Z}$ \\
\hline $\mathrm{C}$ & 2.3320263 & 1.6662651 & 0.7939983 \\
$\mathrm{C}$ & 1.4658168 & -0.6521644 & 0.1897286 \\
$\mathrm{C}$ & 1.2174115 & -1.7324639 & 1.2387408 \\
$\mathrm{C}$ & 1.0111194 & -1.1118307 & 2.6297085 \\
$\mathrm{C}$ & -0.0822569 & -0.0461882 & 2.7779785 \\
$\mathrm{C}$ & 0.1717784 & 1.3733030 & 2.2192928 \\
$\mathrm{C}$ & 1.6343073 & 1.8542740 & 2.1481809 \\
$\mathrm{C}$ & 2.6836586 & 0.2407241 & 0.3295456 \\
$\mathrm{Br}$ & 2.6704962 & -3.0514201 & 1.4368374 \\
$\mathrm{H}$ & 3.2813486 & 2.2253578 & 0.8124669 \\
$\mathrm{H}$ & 1.7143397 & 2.1443236 & 0.0154532 \\
$\mathrm{H}$ & 0.3416939 & -2.3142638 & 0.9269351 \\
$\mathrm{H}$ & 1.9746800 & -0.7124529 & 2.9823280 \\
$\mathrm{H}$ & 0.7671915 & -1.9457075 & 3.3046470 \\
$\mathrm{H}$ & -0.2390503 & 0.0423087 & 3.8653784 \\
$\mathrm{H}$ & -1.0312461 & -0.4332681 & 2.3701697 \\
$\mathrm{H}$ & -0.3906092 & 2.0665790 & 2.8639221 \\
$\mathrm{H}$ & -0.2745029 & 1.4732597 & 1.2188841 \\
$\mathrm{H}$ & 2.2363175 & 1.4036545 & 2.9563352 \\
$\mathrm{H}$ & 1.6438084 & 2.9373888 & 2.3488506
\end{tabular}




$\begin{array}{cccc}\mathrm{H} & 3.4038349 & -0.2324451 & 1.0140789 \\ \mathrm{H} & 3.1762555 & 0.3226875 & -0.6475750 \\ \mathrm{O} & 0.4120538 & -0.2115971 & -0.3319351 \\ \mathrm{Mg} & -0.1017157 & 0.6900782 & -2.0149436 \\ \mathrm{O} & -2.1123815 & 0.4649209 & -1.7345915 \\ \mathrm{Cl} & 0.2713854 & 2.9510978 & -2.3756724 \\ \mathrm{C} & 0.7171249 & -0.9162136 & -3.2997400 \\ \mathrm{H} & 0.7482642 & -0.4120207 & -4.2747500 \\ \mathrm{H} & -0.0605074 & -1.6903987 & -3.2228686 \\ \mathrm{C} & 1.9711241 & -1.2088400 & -2.6976253 \\ \mathrm{H} & 2.8156985 & -0.5835778 & -3.0221130 \\ \mathrm{C} & 2.2152402 & -2.0805522 & -1.6630109 \\ \mathrm{H} & 1.4605867 & -2.8212307 & -1.3815908 \\ \mathrm{H} & 3.2378883 & -2.2623740 & -1.3315306 \\ \mathrm{C} & -3.0006191 & 1.5082913 & -1.2522825 \\ \mathrm{H} & -2.3798747 & 2.3257091 & -0.8639505 \\ \mathrm{H} & -3.5939244 & 1.8720494 & -2.1052649 \\ \mathrm{C} & -2.6304850 & -0.8455018 & -1.3693173 \\ \mathrm{H} & -1.9451166 & -1.2741547 & -0.6224898 \\ \mathrm{H} & -2.6310549 & -1.4716613 & -2.2712756 \\ \mathrm{C} & -4.0155272 & -0.5810299 & -0.7966598 \\ \mathrm{H} & -4.2986797 & -1.3371166 & -0.0532620 \\ \mathrm{H} & -4.7698078 & -0.5798876 & -1.5981782 \\ \mathrm{C} & -3.8632693 & 0.8229944 & -0.2074353 \\ \mathrm{H} & -3.3336954 & 0.7834984 & 0.7572847 \\ \mathrm{H} & -4.8211264 & 1.3395961 & -0.0626828\end{array}$

\section{At $298 \mathrm{~K}$}

Zero Point Energy: $1027.76 \mathrm{~kJ} / \mathrm{mol}$ (ZPE)

Temperature Correction: $53.45 \mathrm{~kJ} / \mathrm{mol}$ (vibration + gas law + rotation + translation)

Enthalpy Correction: $1081.21 \mathrm{~kJ} / \mathrm{mol}$ (ZPE + temperature correction)

Enthalpy: -3971.700777 au (Electronic Energy + Enthalpy Correction)

Entropy: $638.38 \mathrm{~J} / \mathrm{mol} \cdot \mathrm{K}$

Gibbs Energy: -3971.773271 au (Enthalpy - T*Entropy)

Cv: $366.55 \mathrm{~J} / \mathrm{mol} \cdot \mathrm{K}$ 
TS from lowest energy conformer, hindered diastereoface (22'):

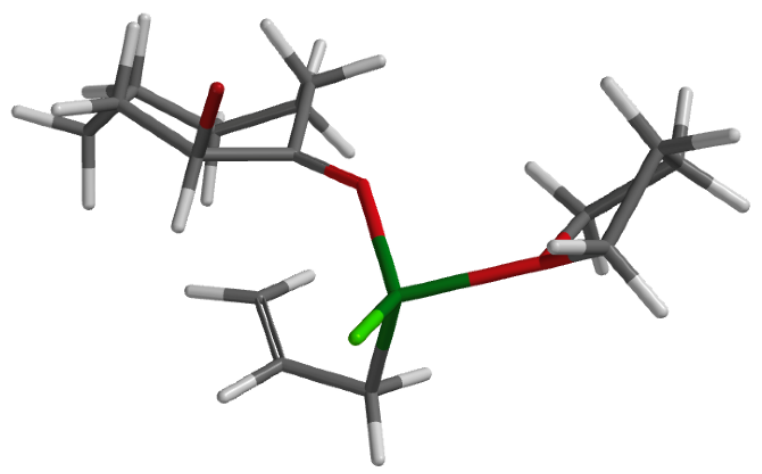

SCF Total Energy: -3972.101858 Hartrees

Two imaginary frequencies: $280 i \mathrm{~cm}^{-1}$, corresponding to $\mathrm{C}-\mathrm{C}$ bond formation; $30 i \mathrm{~cm}^{-1}$, corresponding to bending at $\mathrm{Mg}$

$\mathrm{C}-\mathrm{C}$ bond length: $2.496 \AA$

\begin{tabular}{cccc} 
Atom & $\mathrm{X}$ & $\mathrm{Y}$ & $\mathrm{Z}$ \\
\hline $\mathrm{C}$ & -0.9378099 & -1.4876788 & 2.1097461 \\
$\mathrm{C}$ & 0.4364722 & 0.3194107 & 0.6677470 \\
$\mathrm{C}$ & 1.7917953 & 0.8034494 & 1.2010801 \\
$\mathrm{C}$ & 0.1480107 & -2.2945662 & 2.8345813 \\
$\mathrm{C}$ & -0.6436924 & -0.0466849 & 1.6736329 \\
$\mathrm{Br}$ & 1.7694753 & 2.8023875 & 0.9724986 \\
$\mathrm{H}$ & -1.8052673 & -1.3989107 & 2.7849126 \\
$\mathrm{H}$ & -1.2967028 & -2.0615678 & 1.2406549 \\
$\mathrm{H}$ & 2.5907408 & 0.5009879 & 0.5183472 \\
$\mathrm{H}$ & -0.3770724 & -3.0825383 & 3.3986691 \\
$\mathrm{H}$ & -0.4398278 & 0.5575939 & 2.5729110 \\
$\mathrm{H}$ & -1.5697899 & 0.3522109 & 1.2351944 \\
$\mathrm{O}$ & 0.0491814 & 0.7348944 & -0.4511898 \\
$\mathrm{Mg}$ & 0.7130874 & 0.9820921 & -2.2937325 \\
$\mathrm{C}$ & 1.2347620 & -1.1670874 & -2.5439424 \\
$\mathrm{H}$ & 1.8273779 & -1.0956984 & -3.4648045 \\
$\mathrm{H}$ & 0.2440980 & -1.6259223 & -2.6682259 \\
$\mathrm{C}$ & 1.9316925 & -1.4327291 & -1.3440060 \\
$\mathrm{H}$ & 3.0037675 & -1.1871948 & -1.3467669 \\
$\mathrm{C}$ & 1.3934274 & -1.8391692 & -0.1401755 \\
$\mathrm{H}$ & 2.0655607 & -2.1024751 & 0.6742218 \\
$\mathrm{H}$ & 0.3827385 & -2.2531587 & -0.1161339 \\
$\mathrm{H}$ & 0.7795443 & -2.8309239 & 2.1131499
\end{tabular}




$\begin{array}{llcl}\mathrm{C} & 1.0585088 & -1.5364768 & 3.8067795 \\ \mathrm{H} & 1.4237427 & -2.2703805 & 4.5431391 \\ \mathrm{H} & 0.4757967 & -0.7998098 & 4.3894783 \\ \mathrm{C} & 2.1668141 & 0.5742608 & 2.6677553 \\ \mathrm{H} & 1.4679986 & 1.1349981 & 3.3091593 \\ \mathrm{H} & 3.1407689 & 1.0703614 & 2.7893972 \\ \mathrm{C} & 2.3070823 & -0.8543762 & 3.2202533 \\ \mathrm{H} & 3.0309260 & -0.7655395 & 4.0458185 \\ \mathrm{H} & 2.7898483 & -1.5147180 & 2.4823600 \\ \mathrm{O} & -1.1328293 & 1.2833235 & -3.1191651 \\ \mathrm{Cl} & 2.6458790 & -3.1395624 \\ \mathrm{C} & 2.0837897 & 2.6066025 & -3.2855395 \\ \mathrm{H} & -1.7039399 & 2.7934736 & -4.3625159 \\ \mathrm{H} & -1.8378368 & 3.3326348 & -2.8762088 \\ \mathrm{C} & -0.9893988 & 0.3189336 & -2.8361785 \\ \mathrm{H} & -2.1873819 & -0.4772875 & -3.5901535 \\ \mathrm{H} & -2.1309655 & -0.1011962 & -1.8400172 \\ \mathrm{C} & -1.9837238 & 2.5363863 & -2.5497349 \\ \mathrm{H} & -3.0287841 & 3.3080625 & -2.8892121 \\ \mathrm{H} & -3.7319969 & 2.6549740 & -1.4671769 \\ \mathrm{C} & -2.8668565 & 1.1135703 & -2.8773499 \\ \mathrm{H} & -3.4888572 & 0.7205617 & -2.1644176 \\ \mathrm{H} & -4.2253546 & 1.0790411 & -3.8852776\end{array}$

\section{At 298K}

Zero Point Energy: $1029.09 \mathrm{~kJ} / \mathrm{mol}$ (ZPE)

Temperature Correction: $53.38 \mathrm{~kJ} / \mathrm{mol}$ (vibration + gas law + rotation + translation)

Enthalpy Correction: $1082.47 \mathrm{~kJ} / \mathrm{mol}$ (ZPE + temperature correction)

Enthalpy: -3971.689567 au (Electronic Energy + Enthalpy Correction)

Entropy: $658.90 \mathrm{~J} / \mathrm{mol} \cdot \mathrm{K}$

Gibbs Energy: -3971.764391 au (Enthalpy - T*Entropy)

$\mathrm{Cv}: 366.66 \mathrm{~J} / \mathrm{mol} \cdot \mathrm{K}$ 
TS from Complex II (23'):

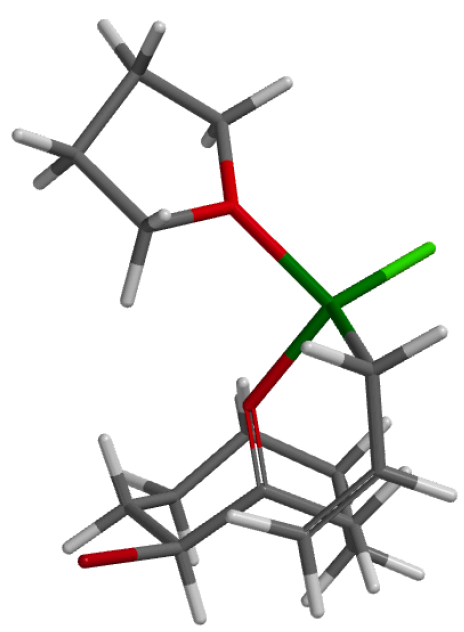

SCF Total Energy: -3972.110408 Hartrees

Two imaginary frequencies: $342 i \mathrm{~cm}^{-1}$, corresponding to $\mathrm{C}-\mathrm{C}$ bond formation; $54 i \mathrm{~cm}^{-1}$, corresponding to bending at $\mathrm{Mg}$ $\mathrm{C}-\mathrm{C}$ bond length: $2.409 \AA$

\begin{tabular}{cccc} 
Atom & $\mathrm{X}$ & $\mathrm{Y}$ & $\mathrm{Z}$ \\
\hline $\mathrm{C}$ & -3.1568081 & 1.3767823 & 0.7644401 \\
$\mathrm{C}$ & -1.4243497 & -0.4896645 & 0.1986440 \\
$\mathrm{C}$ & -1.6479267 & -1.2397248 & 1.5220820 \\
$\mathrm{C}$ & -0.9315807 & -0.5446610 & 2.7005496 \\
$\mathrm{C}$ & -1.3341549 & 0.8796151 & 3.1125994 \\
$\mathrm{C}$ & -1.0238763 & 2.0007909 & 2.1048366 \\
$\mathrm{C}$ & -2.2288229 & 2.4734876 & 1.2797395 \\
$\mathrm{C}$ & -2.5794765 & 0.3948986 & -0.2644247 \\
$\mathrm{Br}$ & -0.9381115 & -3.0866437 & 1.5262845 \\
$\mathrm{H}$ & -4.0204423 & 1.8547923 & 0.2750096 \\
$\mathrm{H}$ & -3.5825324 & 0.8141179 & 1.6114457 \\
$\mathrm{H}$ & -2.7207413 & -1.3702022 & 1.7074097 \\
$\mathrm{H}$ & 0.1447840 & -0.5502346 & 2.4646317 \\
$\mathrm{H}$ & -1.0713652 & -1.1974996 & 3.5744348 \\
$\mathrm{H}$ & -0.7704256 & 1.0709801 & 4.0390452 \\
$\mathrm{H}$ & -2.3963920 & 0.9050161 & 3.4090982 \\
$\mathrm{H}$ & -0.2123356 & 1.6744698 & 1.4371169 \\
$\mathrm{H}$ & -0.6302601 & 2.8736769 & 2.6481974 \\
$\mathrm{H}$ & -1.8704527 & 3.0798152 & 0.4305608 \\
$\mathrm{H}$ & -2.8398990 & 3.1471380 & 1.9048285
\end{tabular}




$\begin{array}{cccc}\mathrm{H} & -2.2326077 & 0.9507654 & -1.1495779 \\ \mathrm{H} & -3.4049187 & -0.2528285 & -0.5838482 \\ \mathrm{O} & -0.2316426 & -0.1981294 & -0.0828290 \\ \mathrm{Mg} & 0.5628042 & 0.4792901 & -1.7634305 \\ \mathrm{Cl} & 2.7681312 & -2.1082207 \\ \mathrm{C} & 0.4488034 & -1.1009984 & -3.1051277 \\ \mathrm{H} & -0.2500427 & -1.8795873 & -2.9313818 \\ \mathrm{H} & 0.5073504 & -0.6238059 & -4.0911908 \\ \mathrm{C} & -0.1870044 & -1.3421214 & -2.5870245 \\ \mathrm{H} & -1.5465992 & -0.7439697 & -3.0207303 \\ \mathrm{C} & -2.3600342 & -2.1378836 & -1.5033705 \\ \mathrm{H} & -1.8602916 & -2.3473264 & -1.2527448 \\ \mathrm{H} & -2.9024757 & -2.8414260 & -1.1340526 \\ \mathrm{O} & -1.1129662 & 0.0083313 & -1.3776745 \\ \mathrm{C} & 2.5015585 & -1.3302127 & -0.9478522 \\ \mathrm{H} & 2.8722191 & -1.9599566 & -1.8470636 \\ \mathrm{H} & 2.9529626 & -1.7122500 & -0.3024250 \\ \mathrm{C} & 2.0695396 & 0.9290080 & -1.1898269 \\ \mathrm{H} & 3.6077185 & 1.5455588 & -0.3026734 \\ \mathrm{H} & 3.3944074 & 1.5713722 & -2.0778357 \\ \mathrm{C} & 3.6625322 & 0.0338744 & -0.9844380 \\ \mathrm{H} & 4.8181114 & 0.5401821 & -0.4181312 \\ \mathrm{H} & 5.6106131 & -0.2833108 & -1.9557554 \\ \mathrm{C} & 5.2281574 & -1.1590006 & -0.2431805 \\ \mathrm{H} & 4.2097377 & -0.9128911 & 0.8186360 \\ \mathrm{H} & 4.0548733 & -2.0677657 & -0.3047795\end{array}$

\section{At $298 \mathrm{~K}$}

Zero Point Energy: $1027.36 \mathrm{~kJ} / \mathrm{mol}(\mathrm{ZPE})$

Temperature Correction: $53.38 \mathrm{~kJ} / \mathrm{mol}$ (vibration + gas law + rotation + translation) Enthalpy Correction: $1080.73 \mathrm{~kJ} / \mathrm{mol}$ (ZPE + temperature correction)

Enthalpy: -3971.698779 au (Electronic Energy + Enthalpy Correction)

Entropy: $676.30 \mathrm{~J} / \mathrm{mol} \cdot \mathrm{K}$

Gibbs Energy: -3971.775580 au (Enthalpy - T*Entropy)

$\mathrm{Cv}: 366.50 \mathrm{~J} / \mathrm{mol} \cdot \mathrm{K}$ 
TS from higher energy conformer (lowest energy conformer, 24'):

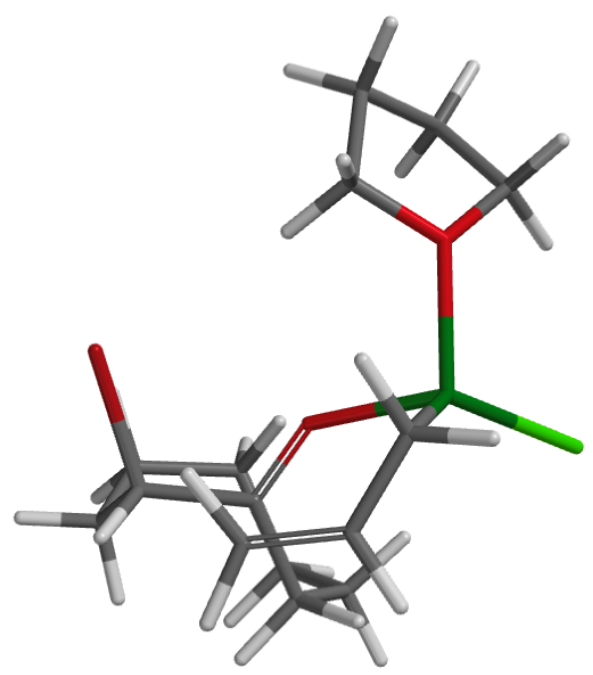

SCF Total Energy: -3972.120451 Hartrees

Two imaginary frequencies: $328 i \mathrm{~cm}^{-1}$, corresponding to $\mathrm{C}-\mathrm{C}$ bond formation; $50 i \mathrm{~cm}^{-1}$, corresponding to bending at $\mathrm{Mg}$ $\mathrm{C}-\mathrm{C}$ bond length: $2.403 \AA$

\begin{tabular}{cccc} 
Atom & $\mathrm{X}$ & $\mathrm{Y}$ & $\mathrm{Z}$ \\
\hline $\mathrm{C}$ & -2.7044998 & 0.6944581 & -0.8953792 \\
$\mathrm{C}$ & -0.9118882 & -1.0712128 & -0.4205466 \\
$\mathrm{C}$ & -1.1536998 & -2.0987615 & 0.6924943 \\
$\mathrm{C}$ & -2.3481175 & -1.7959375 & 1.6056152 \\
$\mathrm{C}$ & -2.3350004 & -0.5166279 & 2.4397385 \\
$\mathrm{C}$ & -2.3115842 & 0.8102201 & 1.6693098 \\
$\mathrm{C}$ & -3.3081710 & 0.9052233 & 0.4975570 \\
$\mathrm{C}$ & -2.1282178 & -0.6886870 & -1.2542199 \\
$\mathrm{Br}$ & 0.4776736 & -2.3804795 & 1.7538186 \\
$\mathrm{H}$ & -1.9096041 & 1.4429729 & -1.0528811 \\
$\mathrm{H}$ & -3.4784576 & 0.9110002 & -1.6491999 \\
$\mathrm{H}$ & -1.3401632 & -3.0738906 & 0.2316432 \\
$\mathrm{H}$ & -2.4572259 & -2.6603832 & 2.2776774 \\
$\mathrm{H}$ & -3.2432275 & -1.8129885 & 0.9621947 \\
$\mathrm{H}$ & -1.4858403 & -0.5443103 & 3.1419344 \\
$\mathrm{H}$ & -3.2460689 & -0.5451362 & 3.0614456 \\
$\mathrm{H}$ & -1.2948350 & 1.0144506 & 1.2993375 \\
$\mathrm{H}$ & -2.5277594 & 1.6085032 & 2.3963780 \\
$\mathrm{H}$ & -3.7566737 & 1.9110587 & 0.4935554 \\
$\mathrm{H}$ & -4.1502037 & 0.2081214 & 0.6560445
\end{tabular}




$\begin{array}{cccc}\mathrm{H} & -1.8033369 & -0.6374821 & -2.3017463 \\ \mathrm{H} & -2.8961269 & -1.4742133 & -1.1944762 \\ \mathrm{O} & 0.0434360 & -0.2627200 & -0.3323940 \\ \mathrm{Mg} & 1.1470842 & 0.6709865 & -1.6861447 \\ \mathrm{O} & 2.6269374 & 1.3315589 & -0.4537422 \\ \mathrm{C} & 1.7759366 & -1.1907612 & -2.7257059 \\ \mathrm{H} & 2.2020762 & -0.7701988 & -3.6457122 \\ \mathrm{H} & 2.5188417 & -1.6001130 & -2.0259942 \\ \mathrm{C} & 0.5501002 & -1.8903992 & -2.8497481 \\ \mathrm{H} & -0.0588954 & -1.6365735 & -3.7295906 \\ \mathrm{C} & -0.0254054 & -2.7275678 & -1.9185430 \\ \mathrm{H} & 0.5882598 & -3.1369196 & -1.1111997 \\ \mathrm{H} & -0.9550455 & -3.2496390 & -2.1556906 \\ \mathrm{Cl} & 0.3101809 & 2.5171378 & -2.8140508 \\ \mathrm{C} & 2.8928582 & 2.7125150 & -0.0984364 \\ \mathrm{H} & 2.0276714 & 3.3095188 & -0.4137023 \\ \mathrm{H} & 3.7883536 & 3.0439912 & -0.6476485 \\ \mathrm{C} & 3.1588406 & 0.4375459 & 0.5672516 \\ \mathrm{H} & 3.8276976 & -0.2808174 & 0.0746276 \\ \mathrm{H} & 2.3054409 & -0.0981093 & 1.0070791 \\ \mathrm{C} & 3.1262932 & 2.6743617 & 1.4005992 \\ \mathrm{H} & 2.1633431 & 2.6568986 & 1.9342553 \\ \mathrm{H} & 3.7066439 & 3.5385911 & 1.7490297 \\ \mathrm{C} & 3.8638005 & 1.3434424 & 1.5700473 \\ \mathrm{H} & 3.8029378 & 0.9411723 & 2.5896887 \\ \mathrm{H} & 4.9256407 & 1.4602006 & 1.3054294\end{array}$

\section{At $298 \mathrm{~K}$}

Zero Point Energy: $1028.63 \mathrm{~kJ} / \mathrm{mol}$ (ZPE)

Temperature Correction: $53.11 \mathrm{~kJ} / \mathrm{mol}$ (vibration + gas law + rotation + translation)

Enthalpy Correction: $1081.74 \mathrm{~kJ} / \mathrm{mol}$ (ZPE + temperature correction)

Enthalpy: -3971.708438 au (Electronic Energy + Enthalpy Correction)

Entropy: $665.23 \mathrm{~J} / \mathrm{mol} \cdot \mathrm{K}$

Gibbs Energy: -3971.783981 au (Enthalpy - T*Entropy)

$\mathrm{Cv}: 365.27 \mathrm{~J} / \mathrm{mol} \cdot \mathrm{K}$ 


\section{Calculation of Ground-State Conformers of Ketone 30}

The approach used in Part A was followed. A conformational search (MMFF) of 10,000 potential conformers produced two low-energy conformers. These two conformers were minimized ( $\omega \mathrm{B} 97 \mathrm{X}-\mathrm{D} / \mathrm{cc}-\mathrm{pVDZ}$ in THF solvent), which produced the conformer shown below as the lowest-energy conformer ( $4.9 \mathrm{kcal} / \mathrm{mol}$ lower in electronic energy than the other).

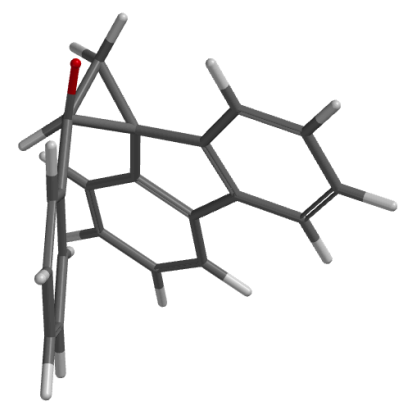

SCF Total Energy: -922.968719 Hartrees

No imaginary frequencies

\begin{tabular}{cccc} 
Atom & $\mathrm{X}$ & $\mathrm{Y}$ & $\mathrm{Z}$ \\
\hline $\mathrm{C}$ & 1.7344471 & -1.0088442 & 0.6429258 \\
$\mathrm{C}$ & 2.7204879 & -3.3042528 & -0.5781481 \\
$\mathrm{C}$ & 3.0743324 & -1.0525545 & 0.2695248 \\
$\mathrm{C}$ & 0.8872823 & -2.1064810 & 0.4051448 \\
$\mathrm{C}$ & 1.3751436 & -3.2607288 & -0.2071379 \\
$\mathrm{C}$ & 3.5624727 & -2.2109374 & -0.3410389 \\
$\mathrm{C}$ & -0.4555838 & -1.7739669 & 0.9055320 \\
$\mathrm{C}$ & -2.7399701 & -0.6879589 & 2.0496056 \\
$\mathrm{C}$ & -1.6274480 & -2.5297475 & 0.9281964 \\
$\mathrm{C}$ & -0.4258000 & -0.4721774 & 1.4433893 \\
$\mathrm{C}$ & -1.5662796 & 0.0713944 & 2.0298530 \\
$\mathrm{C}$ & -2.7729291 & -1.9742931 & 1.5008351 \\
$\mathrm{C}$ & 0.9488368 & 0.0939104 & 1.2715713 \\
$\mathrm{C}$ & 1.1930130 & 1.5341821 & 0.7809219 \\
$\mathrm{C}$ & 1.5440648 & 1.1520410 & 2.1759018 \\
$\mathrm{C}$ & -0.0028264 & 2.3878222 & 0.4837421 \\
$\mathrm{C}$ & -0.5853006 & 2.2858218 & -0.8889567 \\
$\mathrm{C}$ & -1.7764571 & 2.0996999 & -3.4114822 \\
$\mathrm{C}$ & -1.6162625 & 3.1635166 & -1.2501473 \\
$\mathrm{C}$ & -0.1591612 & 1.3097999 & -1.7994112 \\
$\mathrm{C}$ & -0.7559864 & 1.2169410 & -3.0557096 \\
$\mathrm{C}$ & -2.2062404 & 3.0744476 & -2.5074818
\end{tabular}




$\begin{array}{lccc}\mathrm{O} & -0.5017890 & 3.1081640 & 1.3316402 \\ \mathrm{H} & 3.1205751 & -4.1993659 & -1.0580900 \\ \mathrm{H} & 3.7365781 & -0.2018033 & 0.4477933 \\ \mathrm{H} & 0.7193764 & -4.1135846 & -0.3944246 \\ \mathrm{H} & 4.6120034 & -2.2632254 & -0.6369980 \\ \mathrm{H} & -3.6409019 & -0.2708276 & 2.5033656 \\ \mathrm{H} & -1.6483558 & -3.5387868 & 0.5114237 \\ \mathrm{H} & -1.5536112 & 1.0712561 & 2.4654554 \\ \mathrm{H} & -3.6996484 & -2.5508848 & 1.5278471 \\ \mathrm{H} & 2.0025675 & 1.6170407 & 0.0517356 \\ \mathrm{H} & 0.8980545 & 1.5294950 & 2.9709939 \\ \mathrm{H} & 2.6013082 & 1.0537609 & 2.4261351 \\ \mathrm{H} & -2.2429008 & 2.0257588 & -4.3965012 \\ \mathrm{H} & -1.9467667 & 3.9110350 & -0.5280745 \\ \mathrm{H} & 0.6275828 & 0.6040252 & -1.5279078 \\ \mathrm{H} & -0.4254463 & 0.4497884 & -3.7577253 \\ \mathrm{H} & -3.0084618 & 3.7605197 & -2.7842987\end{array}$

Zero Point Energy: 829.48 kJ/mol (ZPE)

Temperature Correction: $40.59 \mathrm{~kJ} / \mathrm{mol}$ (vibration + gas law + rotation + translation) Enthalpy Correction: $870.07 \mathrm{~kJ} / \mathrm{mol}$ (ZPE + temperature correction) Enthalpy: -922.637326 au (Electronic Energy + Enthalpy Correction) Entropy: $553.86 \mathrm{~J} / \mathrm{mol} \cdot \mathrm{K}$ Gibbs Energy: -922.700222 au (Enthalpy - T*Entropy) $\mathrm{Cv}: 292.65 \mathrm{~J} / \mathrm{mol} \cdot \mathrm{K}$ 


\section{Calculation of Ground-State Conformers of Ketone 37}

The approach used in Part A was followed. A conformational search (MMFF) of 10,000 potential conformers produced nine low-energy conformers, which were minimized using B3LYP/6-31G*. The three highest-energy conformers ( $\geq 9 \mathrm{kcal} / \mathrm{mol}$ above the lowest) were not optimized further. The remaining six conformers were then minimized $(\omega \mathrm{B} 97 \mathrm{X}-\mathrm{D} / \mathrm{cc}-\mathrm{pVDZ}$ in THF solvent), which produced the conformer shown below as the lowest-energy conformer (1.8 $\mathrm{kcal} / \mathrm{mol}$ lower in electronic energy than one where the benzoyl group was axial and the SPh group equatorial). The lowest energy conformer shown had a different orientation of the SPh group with respect to the cyclohexane ring compared to the structured determined by X-ray crystallography (40; that conformer was $1.5 \mathrm{kcal} / \mathrm{mol}$ higher in electronic energy than the lowest-energy conformer).

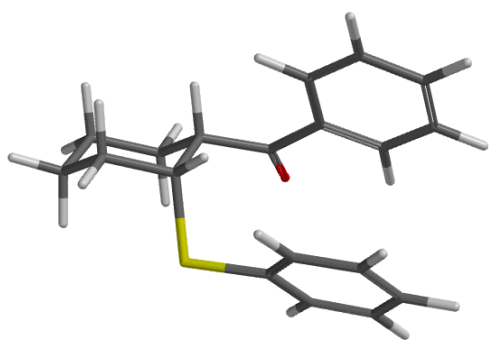

SCF Total Energy: -1209.308768 Hartrees

No imaginary frequencies

\begin{tabular}{cccc} 
Atom & $\mathrm{X}$ & $\mathrm{Y}$ & $\mathrm{Z}$ \\
\hline $\mathrm{S}$ & -1.1694663 & -1.4943410 & 1.3463263 \\
$\mathrm{C}$ & 0.7044875 & 0.7578332 & 2.9584753 \\
$\mathrm{C}$ & 1.5265963 & -0.1565504 & 3.8679287 \\
$\mathrm{C}$ & 0.6256565 & -1.0652978 & 1.1812611 \\
$\mathrm{C}$ & 1.4441197 & -1.9657000 & 2.1144991 \\
$\mathrm{C}$ & 1.2329313 & -1.6322241 & 3.5921482 \\
$\mathrm{C}$ & 0.9347473 & 0.4224258 & 1.4834745 \\
$\mathrm{C}$ & 0.1946221 & 1.3754589 & 0.5551675 \\
$\mathrm{H}$ & 0.9613082 & 1.8125690 & 3.1364894 \\
$\mathrm{H}$ & -0.3678825 & 0.6518437 & 3.1883544 \\
$\mathrm{H}$ & 2.6019003 & 0.0349104 & 3.7002022 \\
$\mathrm{H}$ & 1.3220090 & 0.0830275 & 4.9228761 \\
$\mathrm{H}$ & 0.8869359 & -1.3014943 & 0.1418021 \\
$\mathrm{H}$ & 2.5075614 & -1.8145360 & 1.8588374 \\
$\mathrm{H}$ & 1.2082041 & -3.0205450 & 1.9090647 \\
$\mathrm{H}$ & 1.8769254 & -2.2755522 & 4.2115528
\end{tabular}




$\begin{array}{lccc}\mathrm{H} & 0.1912659 & -1.8612057 & 3.8803982 \\ \mathrm{H} & 2.0128500 & 0.5417497 & 1.2703265 \\ \mathrm{O} & -0.6599147 & 2.1287885 & 0.9897458 \\ \mathrm{C} & 0.5232079 & 1.3789964 & -0.9101817 \\ \mathrm{C} & 1.0143871 & 1.4386269 & -3.6674931 \\ \mathrm{C} & 1.6538027 & 0.7444308 & -1.4429159 \\ \mathrm{C} & -0.3488169 & 2.0539343 & -1.7739527 \\ \mathrm{C} & -0.1084830 & 2.0817574 & -3.1434862 \\ \mathrm{C} & 1.8966916 & 0.7742818 & -2.8148042 \\ \mathrm{H} & 2.3620807 & 0.2264056 & -0.7949427 \\ \mathrm{H} & -1.2269256 & 2.5424670 & -1.3515175 \\ \mathrm{H} & -0.8021546 & 2.6006730 & -3.8073290 \\ \mathrm{H} & 2.7806456 & 0.2780676 & -3.2189704 \\ \mathrm{H} & 1.2035568 & 1.4563549 & -4.7425977 \\ \mathrm{C} & -1.8143197 & -1.0238486 & -0.2570087 \\ \mathrm{C} & -2.9126770 & -0.3214666 & -2.7308019 \\ \mathrm{C} & -2.8633058 & -0.1026287 & -0.3231334 \\ \mathrm{C} & -1.3240271 & -1.6026182 & -1.4338459 \\ \mathrm{C} & -1.8628846 & -1.2394799 & -2.6658541 \\ \mathrm{C} & -3.4156696 & 0.2389803 & -1.5577652 \\ \mathrm{H} & -3.2355537 & 0.3583607 & 0.5929672 \\ \mathrm{H} & -0.5180289 & -2.3377806 & -1.3868566 \\ \mathrm{H} & -1.4661261 & -1.6846249 & -3.5803111 \\ \mathrm{H} & -4.2343147 & 0.9599725 & -1.6008294 \\ \mathrm{H} & -3.3359420 & -0.0420219 & -3.6973005\end{array}$

Zero Point Energy: $908.20 \mathrm{~kJ} / \mathrm{mol}$ (ZPE)

Temperature Correction: $42.34 \mathrm{~kJ} / \mathrm{mol}$ (vibration + gas law + rotation + translation)

Enthalpy Correction: $950.54 \mathrm{~kJ} / \mathrm{mol}$ (ZPE + temperature correction)

Enthalpy: -1208.946725 au (Electronic Energy + Enthalpy Correction)

Entropy: $572.79 \mathrm{~J} / \mathrm{mol} \cdot \mathrm{K}$

Gibbs Energy: -1209.011771 au (Enthalpy - T*Entropy)

$\mathrm{Cv}: 300.02 \mathrm{~J} / \mathrm{mol} \cdot \mathrm{K}$ 


\section{X-ray Crystallographic Data}

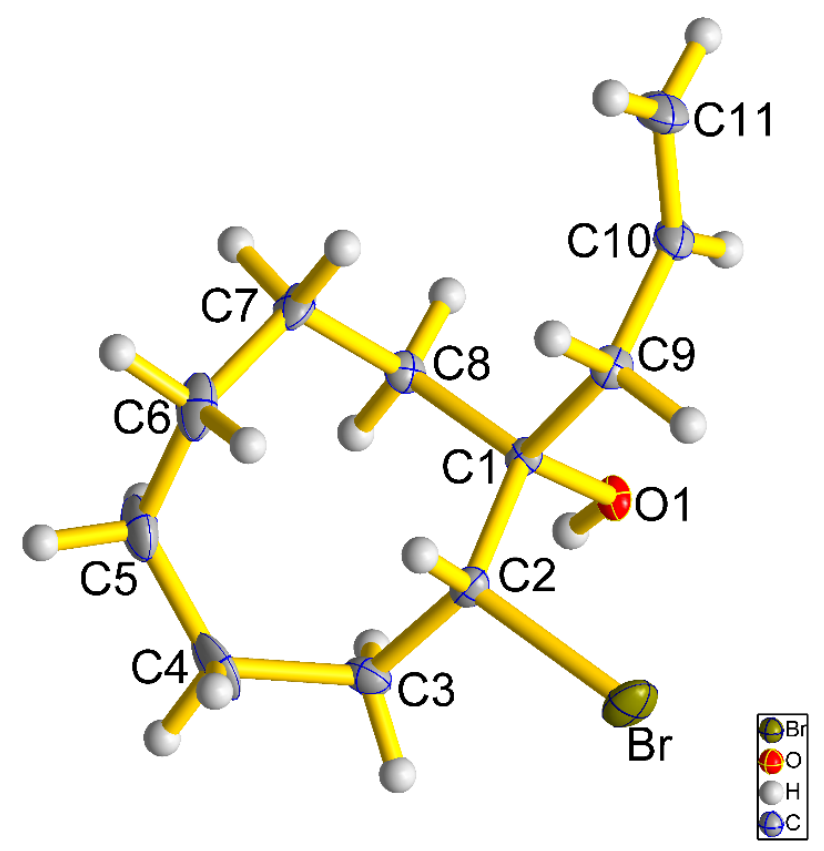

Figure S1. The molecular structure of alcohol anti-10b (ellipsoids set at 50\% probability).

A colorless block-like specimen of $\mathrm{C}_{11} \mathrm{H}_{19} \mathrm{BrO}$, grown by slow evaporation of a solution of alcohol anti-10b in a 5:95 mixture of EtOAc:hexanes, approximate dimensions $0.300 \mathrm{~mm} \times 0.350 \mathrm{~mm}$ $\mathrm{x} 0.460 \mathrm{~mm}$, was used for the X-ray crystallographic analysis. The X-ray intensity data were measured $(\lambda=0.71073 \AA)$.

A total of 1600 frames were collected. The total exposure time was 3.56 hours. The integration of the data using a tetragonal unit cell yielded a total of 36715 reflections to a maximum $\theta$ angle of $28.28^{\circ}$ (0.75 $\AA$ resolution), of which 2897 were independent (average redundancy 12.673, completeness $\left.=100.0 \%, \mathrm{R}_{\text {int }}=4.97 \%, \mathrm{R}_{\text {sig }}=2.20 \%\right)$ and $2740(94.58 \%)$ were greater than $2 \sigma\left(\mathrm{F}^{2}\right)$. The final cell constants of $\underline{\mathrm{a}}=14.2360(17) \AA, \underline{\mathrm{b}}=14.2360(17) \AA, \underline{\mathrm{c}}=11.5095(14) \AA$, volume $=$ 2332.6(6) $\AA^{3}$, are based upon the refinement of the XYZ-centroids of 9911 reflections above 20 $\sigma(\mathrm{I})$ with $4.551^{\circ}<2 \theta<54.36^{\circ}$. Data were corrected for absorption effects using the Multi-Scan method (SADABS). The ratio of minimum to maximum apparent transmission was 0.722 . The calculated minimum and maximum transmission coefficients (based on crystal size) are 0.5382 and 0.7457 .

The structure was solved and refined using the Bruker SHELXTL Software Package, using the space group $\mathrm{P} 42 / \mathrm{n}$, with $\mathrm{Z}=8$ for the formula unit, $\mathrm{C}_{11} \mathrm{H}_{19} \mathrm{BrO}$. The final anisotropic full-matrix least-squares refinement on $\mathrm{F}^{2}$ with 241 variables converged at $\mathrm{R} 1=2.54 \%$, for the observed data and $\mathrm{wR} 2=5.07 \%$ for all data. The goodness-of-fit was 1.127 . The largest peak in the final difference electron density synthesis was $0.254 \mathrm{e}^{-/} / \AA^{3}$ and the largest hole was $-0.259 \mathrm{e}^{-} / \AA^{3}$ with an 
RMS deviation of $0.046 \mathrm{e}^{-} / \AA^{3}$. On the basis of the final model, the calculated density was $1.408 \mathrm{~g} / \mathrm{cm}^{3}$ and $\mathrm{F}(000), 1024 \mathrm{e}^{-}$.

Table S2. Sample and crystal data for alcohol anti-10b.

\begin{tabular}{lll}
\hline Identification code & $20 \mathrm{kaw} 1 \mathrm{~h}$ & \\
Chemical formula & $\mathrm{C}_{11} \mathrm{H}_{19} \mathrm{BrO}$ & \\
Formula weight & $247.17 \mathrm{~g} / \mathrm{mol}$ & \\
Temperature & $100(2) \mathrm{K}$ & \\
Wavelength & $0.71073 \AA$ & \\
Crystal size & $0.300 \times 0.350 \times 0.460 \mathrm{~mm}$ & \\
Crystal habit & colorless block & \\
Crystal system & tetragonal & \\
Space group & $\mathrm{P} 42 / \mathrm{n}$ & $\alpha=90^{\circ}$ \\
Unit cell dimensions & $\mathrm{a}=14.2360(17) \AA$ & $\beta=90^{\circ}$ \\
& $\mathrm{b}=14.2360(17) \AA$ & $\gamma=90^{\circ}$ \\
& $\mathrm{c}=11.5095(14) \AA$ & \\
Volume & $2332.6(6) \AA^{3}$ & \\
Z & 8 & \\
Density (calculated) & $1.408 \mathrm{~g} / \mathrm{cm}^{3}$ & \\
Absorption coefficient & $3.488 \mathrm{~mm}^{-1}$ & \\
F(000) & 1024 & \\
\hline
\end{tabular}

Table S3. Data collection and structure refinement for alcohol anti-10b.

Theta range for data collection

Index ranges

Reflections collected

Independent reflections

Coverage of independent reflections

Absorption correction

Max. and min. transmission

Structure solution technique

Structure solution program

Refinement method

Refinement program

Function minimized

Data / restraints / parameters

Goodness-of-fit on $\mathrm{F}^{2}$

Final $\mathrm{R}$ indices
1.43 to $28.28^{\circ}$

$-18<=\mathrm{h}<=18,-18<=\mathrm{k}<=18,-15<=1<=15$

36715

$2897[\mathrm{R}(\mathrm{int})=0.0497]$

$100.0 \%$

Multi-Scan

0.7457 and 0.5382

direct methods

SHELXT (Sheldrick 2015)

Full-matrix least-squares on $\mathrm{F}^{2}$

SHELXL-2018/3 (Sheldrick, 2018)

$\Sigma \mathrm{w}\left(\mathrm{F}_{\mathrm{o}}^{2}-\mathrm{F}_{\mathrm{c}}^{2}\right)^{2}$

$2897 / 33 / 241$

1.127

2740 data; $\mathrm{I}>2 \sigma(\mathrm{I}) \quad \mathrm{R} 1=0.0254, \mathrm{wR} 2=0.0497$ 
Weighting scheme

Largest diff. peak and hole

R.M.S. deviation from mean

all data $\mathrm{R} 1=0.0283, \mathrm{wR} 2=0.0507$
$\mathrm{w}=1 /\left[\sigma^{2}\left(\mathrm{~F}_{\mathrm{o}}^{2}\right)+(0.0077 \mathrm{P})^{2}+1.1020 \mathrm{P}\right]$
where $\mathrm{P}=\left(\mathrm{F}_{\mathrm{o}}^{2}+2 \mathrm{~F}_{\mathrm{c}}^{2}\right) / 3$
0.254 and $-0.259 \mathrm{e}^{-3}$
$0.046 \mathrm{e} \AA^{-3}$

$\mathrm{R} 1=0.0283, \mathrm{wR} 2=0.0507$

$\mathrm{W}=1 /\left[\sigma^{2}\left(\mathrm{~F}_{\mathrm{o}}{ }^{2}\right)+(0.0077 \mathrm{P})^{2}+1.1020 \mathrm{P}\right]$ where $\mathrm{P}=\left(\mathrm{F}_{\mathrm{o}}^{2}+2 \mathrm{~F}_{\mathrm{c}}^{2}\right) / 3$

$0.046 \mathrm{e}^{-3}$ 


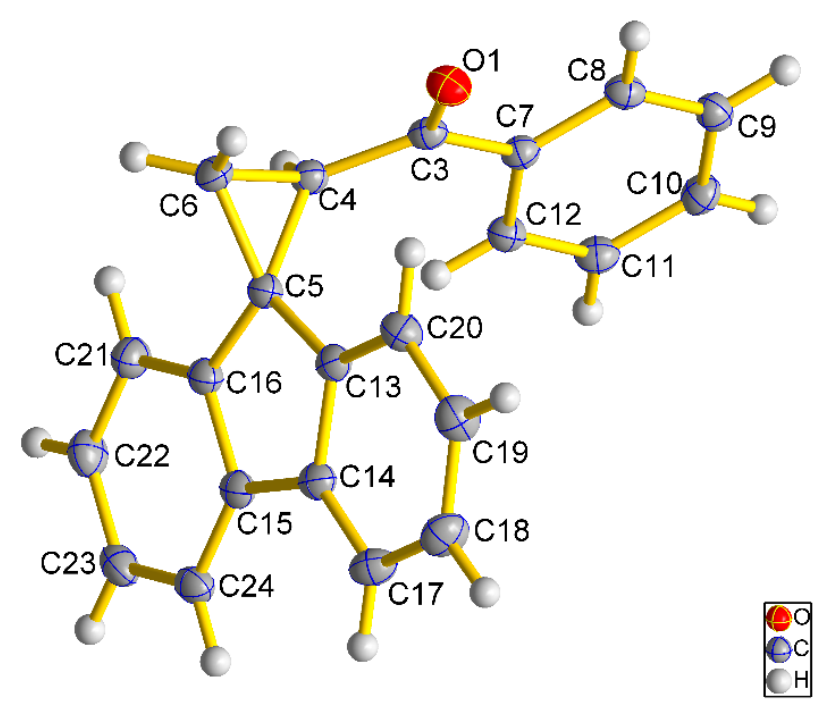

Figure S2. The molecular structure of ketone $\mathbf{3 0}$ (ellipsoids set at 50\% probability).

A colorless prism-like specimen of $\mathrm{C}_{22} \mathrm{H}_{16} \mathrm{O}$, grown by slow evaporation of a solution of ketone 30 in EtOH, approximate dimensions $0.200 \mathrm{~mm}$ x $0.500 \mathrm{~mm}$ x $0.540 \mathrm{~mm}$, was used for the X-ray crystallographic analysis. The X-ray intensity data were measured on a Bruker APEX-II CCD system equipped with a graphite monochromator and a Mo sealed tube $(\lambda=0.71073 \AA)$. A total of 1600 frames were collected. The total exposure time was 3.56 hours. The integration of the data using a monoclinic unit cell yielded a total of 47565 reflections to a maximum $\theta$ angle of $28.29^{\circ}$ ( $0.75 \AA$ resolution), of which 7618 were independent (average redundancy 6.244, completeness $=$ $\left.99.9 \%, \mathrm{R}_{\text {int }}=2.65 \%, \mathrm{R}_{\text {sig }}=1.74 \%\right)$ and $6561(86.12 \%)$ were greater than $2 \sigma\left(\mathrm{F}^{2}\right)$.

The final cell constants of $\underline{\mathrm{a}}=11.4563(5) \AA, \underline{\mathrm{b}}=28.0200(11) \AA, \underline{\mathrm{c}}=9.5696(4) \AA, \beta=93.0703(6)^{\circ}$, volume $=3067.5(2) \AA^{3}$, are based upon the refinement of the XYZ-centroids of reflections above $20 \sigma(\mathrm{I})$. The calculated minimum and maximum transmission coefficients (based on crystal size) are 0.7143 and 0.7457 .

The structure was solved and refined using the Bruker SHELXTL Software Package, using the space group P 121 c 1 , with $\mathrm{Z}=8$ for the formula unit, $\mathrm{C}_{22} \mathrm{H}_{16} \mathrm{O}$. The final anisotropic full-matrix least-squares refinement on $\mathrm{F}^{2}$ with 415 variables converged at $\mathrm{R} 1=4.22 \%$, for the observed data and $\mathrm{wR} 2=12.26 \%$ for all data. The goodness-of-fit was 1.008 . The largest peak in the final difference electron density synthesis was $0.333 \mathrm{e}^{-} / \AA^{3}$ and the largest hole was $-0.223 \mathrm{e}^{-} / \AA^{3}$ with an RMS deviation of $0.045 \mathrm{e}^{-} / \AA^{3}$. On the basis of the final model, the calculated density was $1.283 \mathrm{~g} / \mathrm{cm}^{3}$ and $\mathrm{F}(000), 1248 \mathrm{e}^{-}$.

Table S4. Sample and crystal data for ketone $\mathbf{3 0 .}$

\begin{tabular}{ll}
\hline Identification code & $18 \mathrm{kaw} 3 \mathrm{~h}$ \\
Chemical formula & $\mathrm{C}_{22} \mathrm{H}_{16} \mathrm{O}$
\end{tabular}


Formula weight

Temperature

Wavelength

Crystal size

Crystal habit

Crystal system

Space group

Unit cell dimensions

Volume

Z

Density (calculated)

Absorption coefficient

$\mathrm{F}(000)$
$296.36 \mathrm{~g} / \mathrm{mol}$

100(2) K

$0.71073 \AA$

$0.200 \times 0.500 \times 0.540 \mathrm{~mm}$

colorless prism

monoclinic

P $121 / \mathrm{c} 1$

$\begin{array}{ll}\mathrm{a}=11.4563(5) \AA & \alpha=90^{\circ} \\ \mathrm{b}=28.0200(11) \AA & \beta=93.0703(6)^{\circ} \\ \mathrm{c}=9.5696(4) \AA & \gamma=90^{\circ}\end{array}$

$3067.5(2) \AA^{3}$

8

$1.283 \mathrm{~g} / \mathrm{cm}^{3}$

$0.077 \mathrm{~mm}^{-1}$

1248

Table S5. Data collection and structure refinement for ketone $\mathbf{3 0 .}$

\begin{tabular}{ll}
\hline Diffractometer & Bruker APEX-II CCD \\
Radiation source & sealed tube, Mo \\
Theta range for data collection & 1.45 to $28.29^{\circ}$ \\
Index ranges & $-15<=\mathrm{h}<=15,-37<=\mathrm{k}<=37,-12<=1<=12$ \\
Reflections collected & 47565 \\
Independent reflections & $7618[\mathrm{R}(\mathrm{int})=0.0265]$ \\
Absorption correction & multi-scan \\
Max. and min. transmission & 0.7457 and 0.7143 \\
Structure solution technique & direct methods \\
Structure solution program & SHELXT (Sheldrick 2015) \\
Refinement method & Full-matrix least-squares on $\mathrm{F}^{2}$ \\
Refinement program & $\mathrm{SHELXL-2018/3} \mathrm{(Sheldrick,} \mathrm{2018)}$ \\
Function minimized & $\Sigma \mathrm{w}\left(\mathrm{F}_{\mathrm{o}}^{2}-\mathrm{F}_{\mathrm{c}}^{2}\right)^{2}$ \\
Data / restraints / parameters & $7618 / 0 / 415$ \\
Goodness-of-fit on $\mathrm{F}^{2}$ & 1.008 \\
$\Delta / \sigma_{\text {max }}$ & 0.001
\end{tabular}


Final R indices

Weighting scheme

Largest diff. peak and hole

R.M.S. deviation from mean
6561 data; $\mathrm{I}>2 \sigma(\mathrm{I}) \quad \mathrm{R} 1=0.0422$

$\mathrm{wR} 2=0.1162$

all data

$\mathrm{R} 1=0.0492$

$\mathrm{wR} 2=0.1226$

$\mathrm{W}=1 /\left[\sigma^{2}\left(\mathrm{~F}_{\mathrm{o}}^{2}\right)+(0.0759 \mathrm{P})^{2}+0.8365 \mathrm{P}\right]$

where $\mathrm{P}=\left(\mathrm{F}_{\mathrm{o}}{ }^{2}+2 \mathrm{~F}_{\mathrm{c}}{ }^{2}\right) / 3$

0.333 and $-0.223 \mathrm{e}^{-3}$

$0.045 \mathrm{e}^{-3}$ 


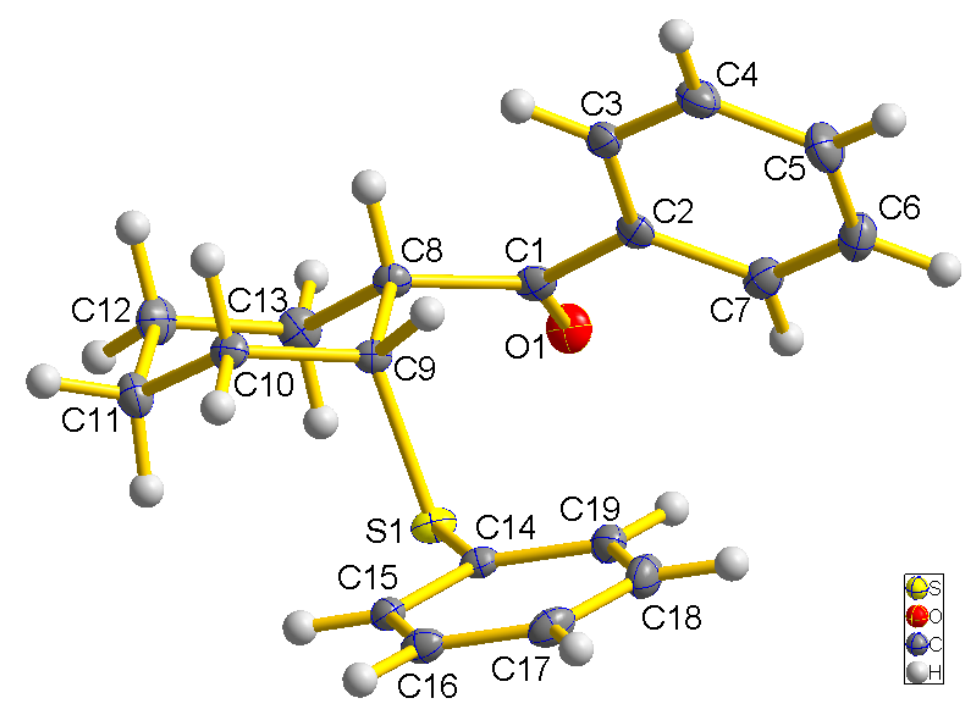

Figure S3. The molecular structure of ketone 37 (ellipsoids set at 50\% probability).

A colorless plate-like specimen of $\mathrm{C}_{19} \mathrm{H}_{20} \mathrm{OS}$, grown by slow evaporation of a solution of ketone 37 in a 5:95 mixture of EtOAc:hexanes, approximate dimensions $0.060 \mathrm{~mm} \times 0.170 \mathrm{~mm} \times 0.460$ $\mathrm{mm}$, was used for the X-ray crystallographic analysis. The X-ray intensity data were measured on a Bruker APEX-II CCD system equipped with a graphite monochromator and a Mo sealed tube $(\lambda$ $=0.71073 \AA$ ).

A total of 1600 frames were collected. The total exposure time was 4.44 hours. The frames were integrated with the Bruker SAINT software package using a narrow-frame algorithm. The integration of the data using a monoclinic unit cell yielded a total of 23748 reflections to a maximum $\theta$ angle of $28.29^{\circ}(0.75 \AA$ resolution), of which 3860 were independent (average redundancy 6.152 , completeness $\left.=99.9 \%, \mathrm{R}_{\text {int }}=4.01 \%, \mathrm{R}_{\text {sig }}=2.70 \%\right)$ and $3230(83.68 \%)$ were greater than $2 \sigma\left(\mathrm{F}^{2}\right)$. The final cell constants of $\underline{\mathrm{a}}=10.1921(8) \AA, \underline{\mathrm{b}}=10.4849(8) \AA$, $\underline{\mathrm{c}}=14.7552(12) \AA, \beta=99.2609(12)^{\circ}$, volume $=1556.2(2) \AA^{3}$, are based upon the refinement of the XYZ-centroids of 5973 reflections above $20 \sigma(\mathrm{I})$ with $4.535^{\circ}<2 \theta<56.30^{\circ}$. Data were corrected for absorption effects using the multi-scan method (SADABS). The ratio of minimum to maximum apparent transmission was 0.945. The calculated minimum and maximum transmission coefficients (based on crystal size) are 0.7050 and 0.7457 .

The structure was solved and refined using the Bruker SHELXTL Software Package, using the space group $\mathrm{P} 121 / \mathrm{n} 1$, with $\mathrm{Z}=4$ for the formula unit, $\mathrm{C}_{19} \mathrm{H}_{20} \mathrm{OS}$. The final anisotropic full-matrix least-squares refinement on $\mathrm{F}^{2}$ with 190 variables converged at $\mathrm{R} 1=3.37 \%$, for the observed data and $\mathrm{wR}_{2}=8.77 \%$ for all data. The goodness-of-fit was 1.039 . The largest peak in the final difference electron density synthesis was $0.369 \mathrm{e}^{-/} / \AA^{3}$ and the largest hole was $-0.268 \mathrm{e}^{-} / \AA^{3}$ with an RMS deviation of $0.047 \mathrm{e}^{-} / \AA^{3}$. On the basis of the final model, the calculated density was $1.265 \mathrm{~g} / \mathrm{cm}^{3}$ and $\mathrm{F}(000), 632 \mathrm{e}^{-}$. 
Table S6. Sample and crystal data for ketone 37.

\begin{tabular}{lll}
\hline Identification code & $15 \mathrm{kaw} 5 \mathrm{~h}$ & \\
Chemical formula & $\mathrm{C}_{19} \mathrm{H}_{20} \mathrm{OS}$ & \\
Formula weight & $296.41 \mathrm{~g} / \mathrm{mol}$ & \\
Temperature & $100(2) \mathrm{K}$ & \\
Wavelength & $0.71073 \AA$ \\
Crystal size & $0.060 \times 0.170 \times 0.460 \mathrm{~mm}$ \\
Crystal habit & colorless plate & \\
Crystal system & monoclinic & \\
Space group & $\mathrm{P} 121 / \mathrm{n} 1$ & \\
Unit cell dimensions & $\mathrm{a}=10.1921(8) \AA$ & $\alpha=90^{\circ}$ \\
& $\mathrm{b}=10.4849(8) \AA$ & $\beta=99.2609(12)^{\circ}$ \\
& $\mathrm{c}=14.7552(12) \AA$ & $\gamma=90^{\circ}$ \\
Volume & $1556.2(2) \AA^{3}$ & \\
Z & 4 & \\
Density (calculated) & $1.265 \mathrm{~g}^{-\mathrm{cm}^{3}}$ & \\
Absorption coefficient & $0.204 \mathrm{~mm}^{-1}$ & \\
F(000) & 632 & \\
\hline
\end{tabular}

Table S7. Data collection and structure refinement for ketone 37 .

\begin{tabular}{ll}
\hline Diffractometer & Bruker APEX-II CCD \\
Radiation source & sealed tube, Mo \\
Theta range for data collection & 2.27 to $28.29^{\circ}$ \\
Index ranges & $-13<=\mathrm{h}<=13,-13<=\mathrm{k}<=13,-19<=1<=19$ \\
Reflections collected & 23748 \\
Independent reflections & $3860[\mathrm{R}(\mathrm{int})=0.0401]$ \\
Coverage of independent reflections & $99.9 \%$ \\
Absorption correction & multi-scan \\
Max. and min. transmission & 0.7457 and 0.7050 \\
Structure solution technique & direct methods \\
Structure solution program & SHELXT (Sheldrick 2014) \\
Refinement method & Full-matrix least-squares on $\mathrm{F}^{2}$ \\
Refinement program & $\mathrm{SHELXL}-2014 / 6$ (Sheldrick, 2014) \\
Function minimized & $\Sigma \mathrm{w}\left(\mathrm{F}_{\mathrm{o}}{ }^{2}-\mathrm{F}_{\mathrm{c}}{ }^{2}\right)^{2}$ \\
Data / restraints / parameters & $3860 / 0 / 190$ \\
Goodness-of-fit on $\mathrm{F}^{2}$ & 1.039 \\
$\Delta / \sigma_{\text {max }}$ & 0.001
\end{tabular}


Final R indices

Weighting scheme

Largest diff. peak and hole

R.M.S. deviation from mean
3230 data; $\mathrm{I}>2 \sigma(\mathrm{I}) \quad \mathrm{R} 1=0.0337, \mathrm{wR} 2=0.0814$

all data

$\mathrm{R} 1=0.0435, \mathrm{wR} 2=0.0877$

$\mathrm{w}=1 /\left[\sigma^{2}\left(\mathrm{~F}_{\mathrm{o}}^{2}\right)+(0.0400 \mathrm{P})^{2}+0.5642 \mathrm{P}\right]$

where $\mathrm{P}=\left(\mathrm{F}_{\mathrm{o}}{ }^{2}+2 \mathrm{~F}_{\mathrm{c}}^{2}\right) / 3$

0.369 and $-0.268 \mathrm{e}^{-3}$

$0.047 \mathrm{e} \AA^{-3}$ 


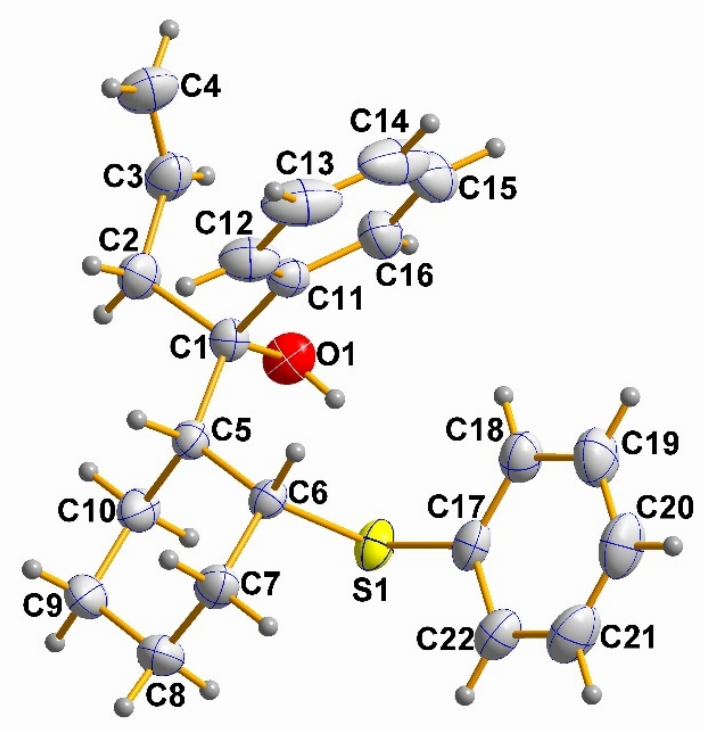

Figure S4. The molecular structure of alcohol $\mathbf{3 8}$ (ellipsoids set at 50\% probability).

A colorless plate-like specimen of $\mathrm{C}_{22} \mathrm{H}_{26} \mathrm{OS}$, grown by slow evaporation of a solution of alcohol 38 in a 20:80 mixture of EtOAc:hexanes, approximate dimensions $0.220 \mathrm{~mm} \times 0.500 \mathrm{~mm}$ $\mathrm{x} 0.560 \mathrm{~mm}$, was used for the X-ray crystallographic analysis. The X-ray intensity data were measured on a Bruker APEX-II CCD system equipped with a graphite monochromator and a Mo sealed tube $(\lambda=0.71073 \AA)$.

The total exposure time was 2.21 hours. The frames were integrated with the Bruker SAINT software package using a narrow-frame algorithm. The integration of the data using a triclinic unit cell yielded a total of 41224 reflections to a maximum $\theta$ angle of $28.33^{\circ}$ ( $0.75 \AA$ resolution), of which 9511 were independent (average redundancy 4.334 , completeness $=99.8 \%, R_{\text {int }}=1.86 \%$, $\left.\mathrm{R}_{\text {sig }}=1.46 \%\right)$ and $8084(85.00 \%)$ were greater than $2 \sigma\left(\mathrm{F}^{2}\right)$. The final cell constants of $\underline{a}=10.5498(4) \AA, \underline{b}=12.2723(5) \AA, \underline{c}=15.3144(6) \AA, \alpha=87.9725(7)^{\circ}, \beta=88.6541(7)^{\circ}, \gamma$ $=74.5755(7)^{\circ}$, volume $=1909.94(13) \AA^{3}$, are based upon the refinement of the XYZ-centroids of 6063 reflections above $20 \sigma(\mathrm{I})$ with $4.414^{\circ}<2 \theta<56.53^{\circ}$. Data were corrected for absorption effects using the multi-scan method (SADABS). The ratio of minimum to maximum apparent transmission was 0.949 . The calculated minimum and maximum transmission coefficients (based on crystal size) are 0.7171 and 0.7457 .

The structure was solved and refined using the Bruker SHELXTL Software Package, using the space group $\mathrm{P}-1$, with $\mathrm{Z}=4$ for the formula unit, $\mathrm{C}_{22} \mathrm{H}_{26} \mathrm{OS}$. The final anisotropic full-matrix least-squares refinement on $\mathrm{F}^{2}$ with 441 variables converged at $\mathrm{R} 1=3.96 \%$, for the observed data and $\mathrm{wR}_{2}=11.37 \%$ for all data. The goodness-of-fit was 1.033 . The largest peak in the final difference electron density synthesis was $0.499 \mathrm{e}^{-/} / \AA^{3}$ and the largest hole was $-0.213 \mathrm{e}^{-} / \AA^{3}$ with an RMS deviation of $0.038 \mathrm{e}^{-} / \AA^{3}$. On the basis of the final model, the calculated density was $1.177 \mathrm{~g} / \mathrm{cm}^{3}$ and $\mathrm{F}(000), 728 \mathrm{e}^{-}$. 
Table S8. Sample and crystal data for alcohol 38.

\begin{tabular}{lll}
\hline Identification code & $15 \mathrm{kaw} 6 \mathrm{~h}$ & \\
Chemical formula & $\mathrm{C}_{22} \mathrm{H}_{26} \mathrm{OS}$ & \\
Formula weight & $338.51 \mathrm{~g} / \mathrm{mol}$ & \\
Temperature & $200(2) \mathrm{K}$ & \\
Wavelength & $0.71073 \AA$ & \\
Crystal size & $0.220 \times 0.500 \times 0.560 \mathrm{~mm}$ & \\
Crystal habit & Colorless plate & \\
Crystal system & triclinic & \\
Space group & $\mathrm{P}-1$ & $\alpha=87.9725(7)^{\circ}$ \\
Unit cell dimensions & $\mathrm{a}=10.5498(4) \AA$ & $\beta=88.6541(7)^{\circ}$ \\
& $\mathrm{b}=12.2723(5) \AA$ & $\gamma=74.5755(7)^{\circ}$ \\
& $\mathrm{c}=15.3144(6) \AA$ & \\
Volume & $1909.94(13) \AA \AA^{3}$ & \\
Z & 4 & \\
Density (calculated) & $1.177 \mathrm{~g} / \mathrm{cm}^{3}$ & \\
Absorption coefficient & $0.175 \mathrm{~mm}^{-1}$ & \\
F(000) & 728 & \\
\hline
\end{tabular}

Table S9. Data collection and structure refinement for alcohol 38.

\begin{tabular}{ll}
\hline Diffractometer & $\begin{array}{l}\text { Bruker APEX-II CCD } \\
\text { sealed tube, Mo }\end{array}$ \\
Radiation source & 1.33 to $28.33^{\circ}$ \\
Theta range for data collection & $-14<=\mathrm{h}<=14,-16<=\mathrm{k}<=16,-20<=1<=20$ \\
Index ranges & 41224 \\
Reflections collected & $9511[\mathrm{R}(\mathrm{int})=0.0186]$ \\
Independent reflections & $99.8 \%$ \\
Coverage of independent reflections & multi-scan \\
Absorption correction & 0.7457 and 0.7171 \\
Max. and min. transmission & direct methods \\
Structure solution technique & SHELXT (Sheldrick 2014) \\
Structure solution program & Full-matrix least-squares on $\mathrm{F}^{2}$ \\
Refinement method & $\mathrm{SHELXL}-2014$ (Sheldrick, 2014) \\
Refinement program & $\Sigma \mathrm{w}\left(\mathrm{F}_{\mathrm{o}}^{2}-\mathrm{F}_{\mathrm{c}}{ }^{2}\right)^{2}$
\end{tabular}


Data / restraints / parameters

Goodness-of-fit on $\mathrm{F}^{2}$

Final $\mathrm{R}$ indices

Weighting scheme

Largest diff. peak and hole

R.M.S. deviation from mean
$9511 / 2 / 441$

1.033

8084 data; $\mathrm{I}>2 \sigma(\mathrm{I}) \quad \mathrm{R} 1=0.0396, \mathrm{wR} 2=0.1068$

all data $\quad \mathrm{R} 1=0.0473, \mathrm{wR} 2=0.1137$

$\mathrm{W}=1 /\left[\sigma^{2}\left(\mathrm{~F}_{\mathrm{o}}^{2}\right)+(0.0575 \mathrm{P})^{2}+0.5046 \mathrm{P}\right]$

where $\mathrm{P}=\left(\mathrm{F}_{\mathrm{o}}^{2}+2 \mathrm{~F}_{\mathrm{c}}{ }^{2}\right) / 3$

0.499 and $-0.213 \mathrm{e} \AA^{-3}$

$0.038 \mathrm{e}^{-3}$ 


\section{References}

(1) Haasnoot, C. A. G. Conformational Analysis of Six-Membered Rings in Solution: Ring Puckering Coordinates Derived from Vicinal NMR Proton-Proton Coupling Constants. $J$. Am. Chem. Soc. 1993, 115, 1460-1468.

(2) Bushweller, C. H.; O'Neil, J. W. The $A$ Value of the Carbodiimido Group. Conformational Requirements of "sp²-Hybridized" Lone Pairs. J. Org. Chem. 1970, 35, 276-278. Schneider, H. J.; Hoppen, V. Carbon-13 Nuclear Magnetic Resonance Substituent-Induced Shieldings and Conformational Equilibriums in Cyclohexanes. J. Org. Chem. 1978, 43, 3866-3873.

Källström, S.; Jagt, R. B. C.; Sillanpää, R.; Feringa, B. L.; Minnaard, A. J.; Leino, R. Highly Enantio- and Diastereoselective One-Pot Reactions in Aqueous Media: Combined Asymmetric Rh-Catalyzed Conjugate Addition/Metal-Mediated Allylation. Eur. J. Org. Chem. 2006, 2006, 3826-3833.

Mehta, G.; Likhite, N. S. Synthetic Studies Towards Bioactive Frondosins: Rapid Framework Access and Diversity Creation. Tetrahedron Lett. 2009, 50, 5263-5266.

Reddy, C.; Babu, S. A.; Aslam, N. A.; Rajkumar, V. Construction of Functionalized Carbocycles Having Contiguous Tertiary Carbinol and All-Carbon Stereogenic Centers. Eur. J. Org. Chem. 2013, 2362-2380.

Hugelshofer, C. L.; Magauer, T. A Bioinspired Cyclization Sequence Enables the Asymmetric Total Synthesis of Dictyoxetane. J. Am. Chem. Soc. 2016, 138, 6420-6423.

Rozada, T. C.; Gauze, G. F.; Rosa, F. A.; Favaro, D. C.; Rittner, R.; Pontes, R. M.; Basso, E. A. The Conformational Analysis of 2-Halocyclooctanones. Spectrochim. Acta, Part A 2015, 137, 176-184.

Shao, Y.; Molnar, L. F.; Jung, Y.; Kussmann, J.; Ochsenfeld, C.; Brown, S. T.; Gilbert, A. T. B.; Slipchenko, L. V.; Levchenko, S. V.; O’Neill, D. P.; DiStasio Jr., R. A.; Lochan, R. C.; Wang, T.; Beran, G. J. O.; Besley, N. A.; Herbert, J. M.; Yeh Lin, C.; Van Voorhis, T.; Hung Chien, S.; Sodt, A.; Steele, R. P.; Rassolov, V. A.; Maslen, P. E.; Korambath, P. P.; Adamson, R. D.; Austin, B.; Baker, J.; Byrd, E. F. C.; Dachsel, H.; Doerksen, R. J.; Dreuw, A.; Dunietz, B. D.; Dutoi, A. D.; Furlani, T. R.; Gwaltney, S. R.; Heyden, A.; Hirata, S.; Hsu, C.-P.; Kedziora, G.; Khalliulin, R. Z.; Klunzinger, P.; Lee, A. M.; Lee, M. S.; Liang, W.; Lotan, I.; Nair, N.; Peters, B.; Proynov, E. I.; Pieniazek, P. A.; Min Rhee, Y.; Ritchie, J.; Rosta, E.; David Sherrill, C.; Simmonett, A. C.; Subotnik, J. E.; Lee Woodcock III, H.; Zhang, W.; Bell, A. T.; Chakraborty, A. K.; Chipman, D. M.; Keil, F. J.; Warshel, A.; Hehre, W. J.; Schaefer III, H. F.; Kong, J.; Krylov, A. I.; Gill, P. M. W.; Head-Gordon, M. Advances in Methods and Algorithms in a Modern Quantum Chemistry Program Package. Phys. Chem. Chem. Phys. 2006, 8, 3172-3191.

(10) Peltzer, R. M.; Gauss, J.; Eisenstein, O.; Cascella, M. The Grignard Reaction - Unraveling a Chemical Puzzle. J. Am. Chem. Soc. 2020, 142, 2984-2994.

(11) Chai, J.-D.; Head-Gordon, M. Long-Range Corrected Hybrid Density Functionals with Damped Atom-Atom Dispersion Corrections. Phys. Chem. Chem. Phys. 2008, 10, 66156620 .

(12) Tomasi, J.; Persico, M. Molecular Interactions in Solution: An Overview of Methods Based on Continuous Distributions of the Solvent. Chem. Rev. 1994, 94, 2027-2094.

(13) Sousa, S. F.; Fernandes, P. A.; Ramos, M. J. Computational Enzymatic Catalysis Clarifying Enzymatic Mechanisms with the Help of Computers. Phys. Chem. Chem. Phys. 2012, 14, 12431-12441. 
(14) Ochterski, J. W. Vibrational Analysis in Gaussian. https://gaussian.com/vib/ (accessed January 5, 2021). 


\section{Selected ${ }^{1} \mathrm{H}$ and ${ }^{13} \mathrm{C}\left\{{ }^{1} \mathrm{H}\right\}$ NMR Spectra}

The following pages contain ${ }^{1} \mathrm{H}$ and ${ }^{13} \mathrm{C}\left\{{ }^{1} \mathrm{H}\right\}$ NMR spectra for all new compounds. Unpurified ${ }^{1} \mathrm{H}$ or ${ }^{13} \mathrm{C}\left\{{ }^{1} \mathrm{H}\right\}$ NMR spectra are also included if they were used to calculate diastereomeric ratios or product ratios in competition experiments. There are no resonances with chemical shifts beyond the shown area of the spectra. 

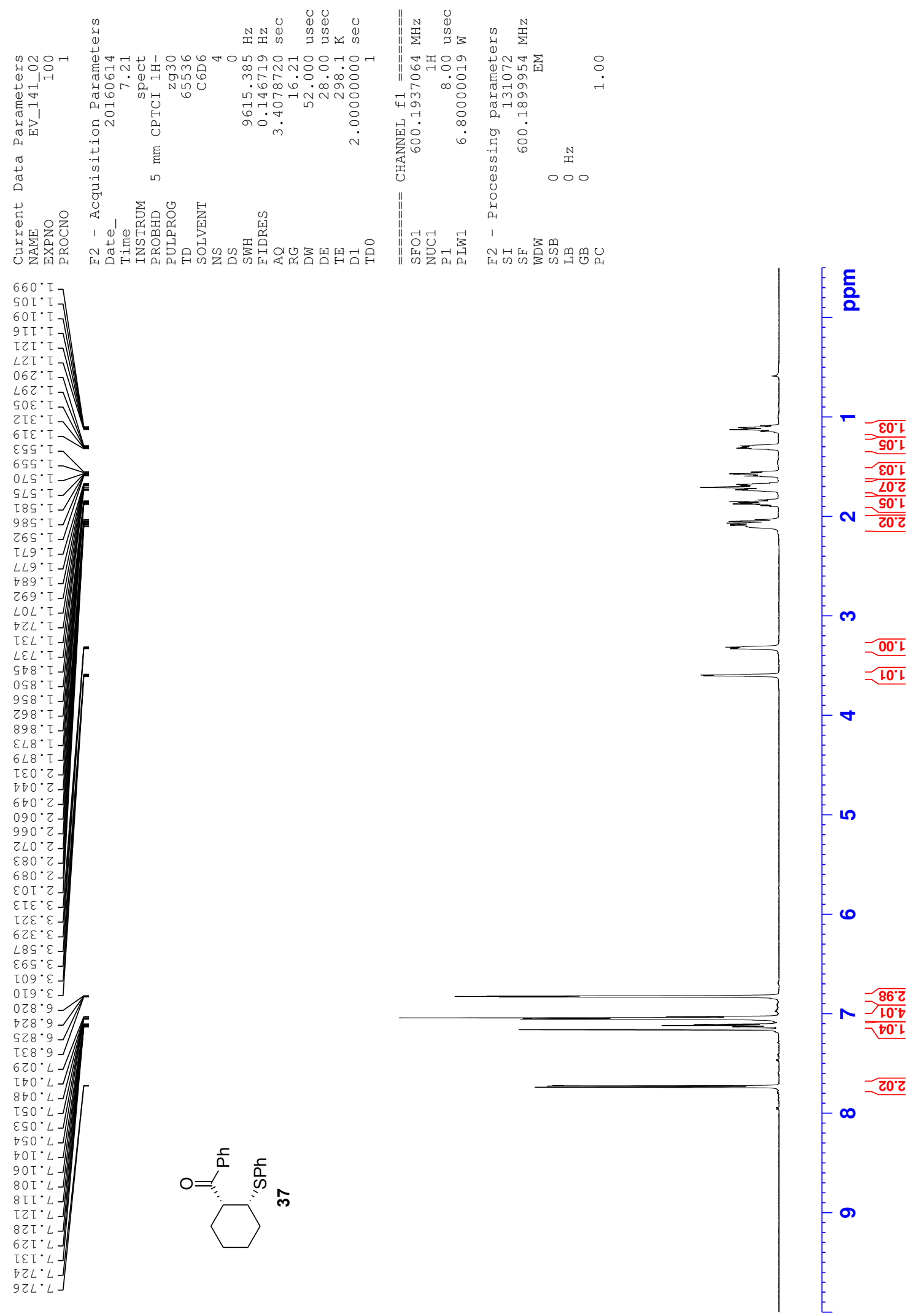

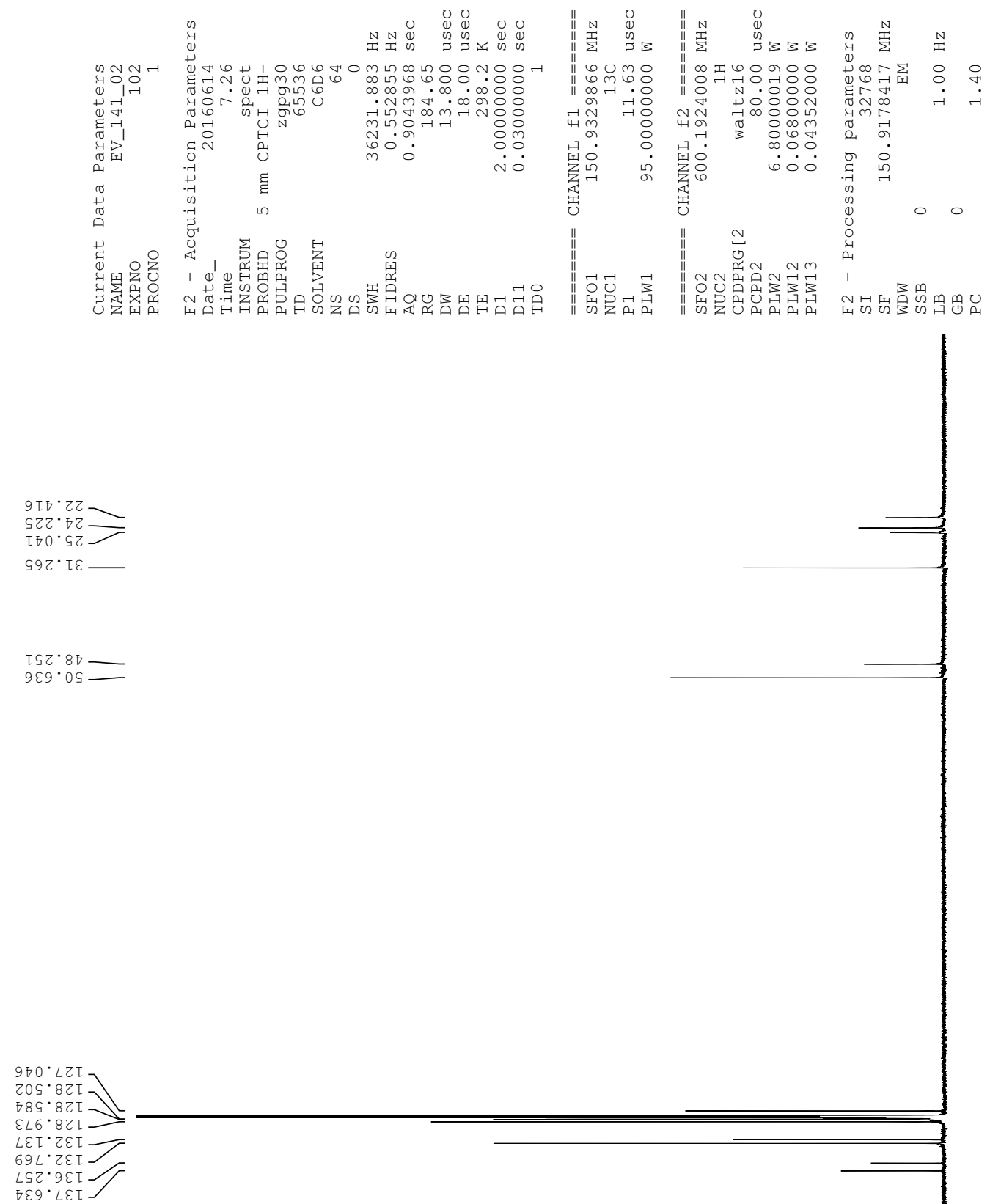

틀

$-\frac{\text { ก }}{2}$

우

8

๕

움

ㅇำ

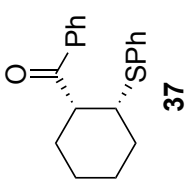

$\stackrel{\circ}{\circ}$

$\stackrel{\circ}{\check{\infty}}$

IZT.00Z

ฉั 

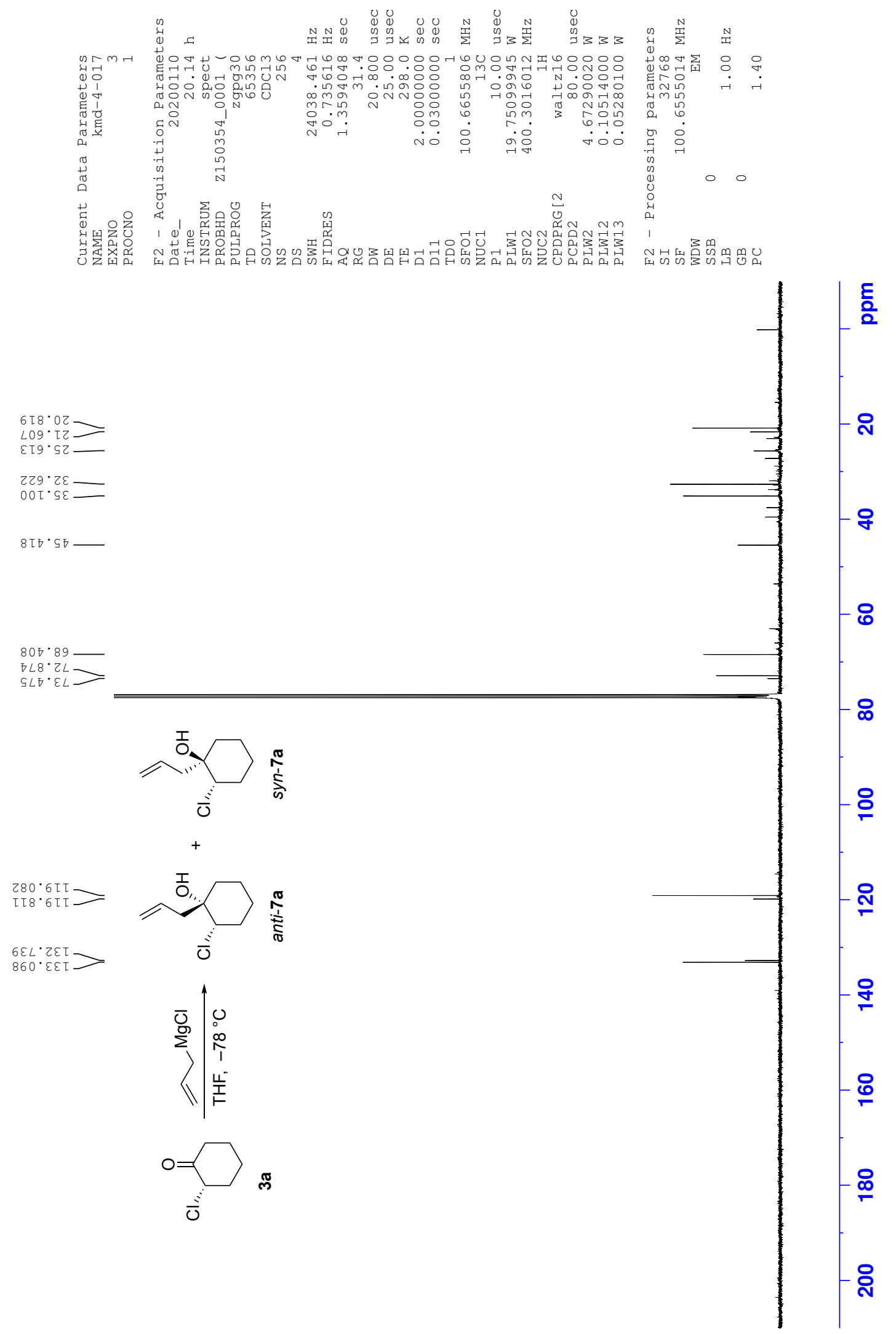

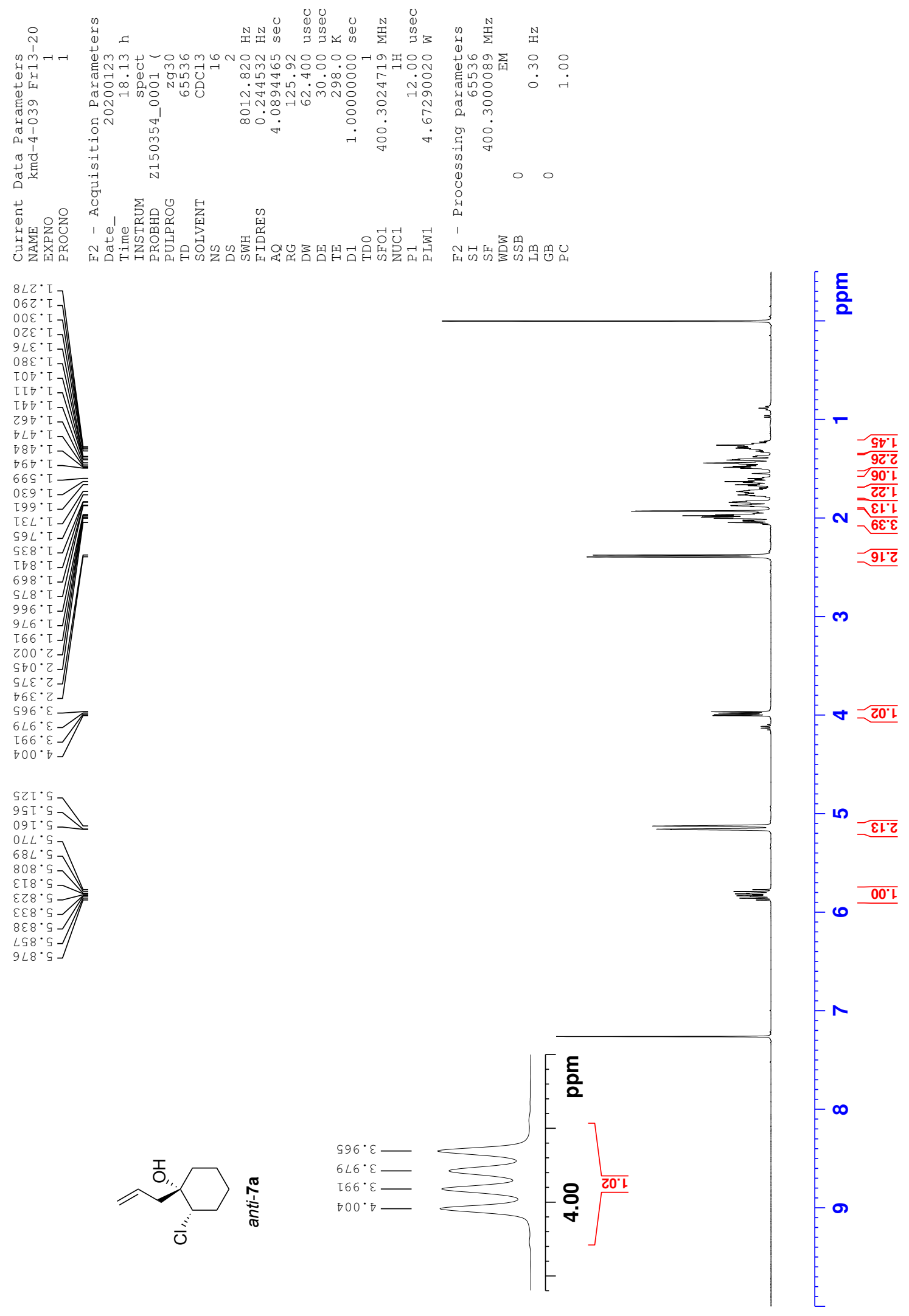

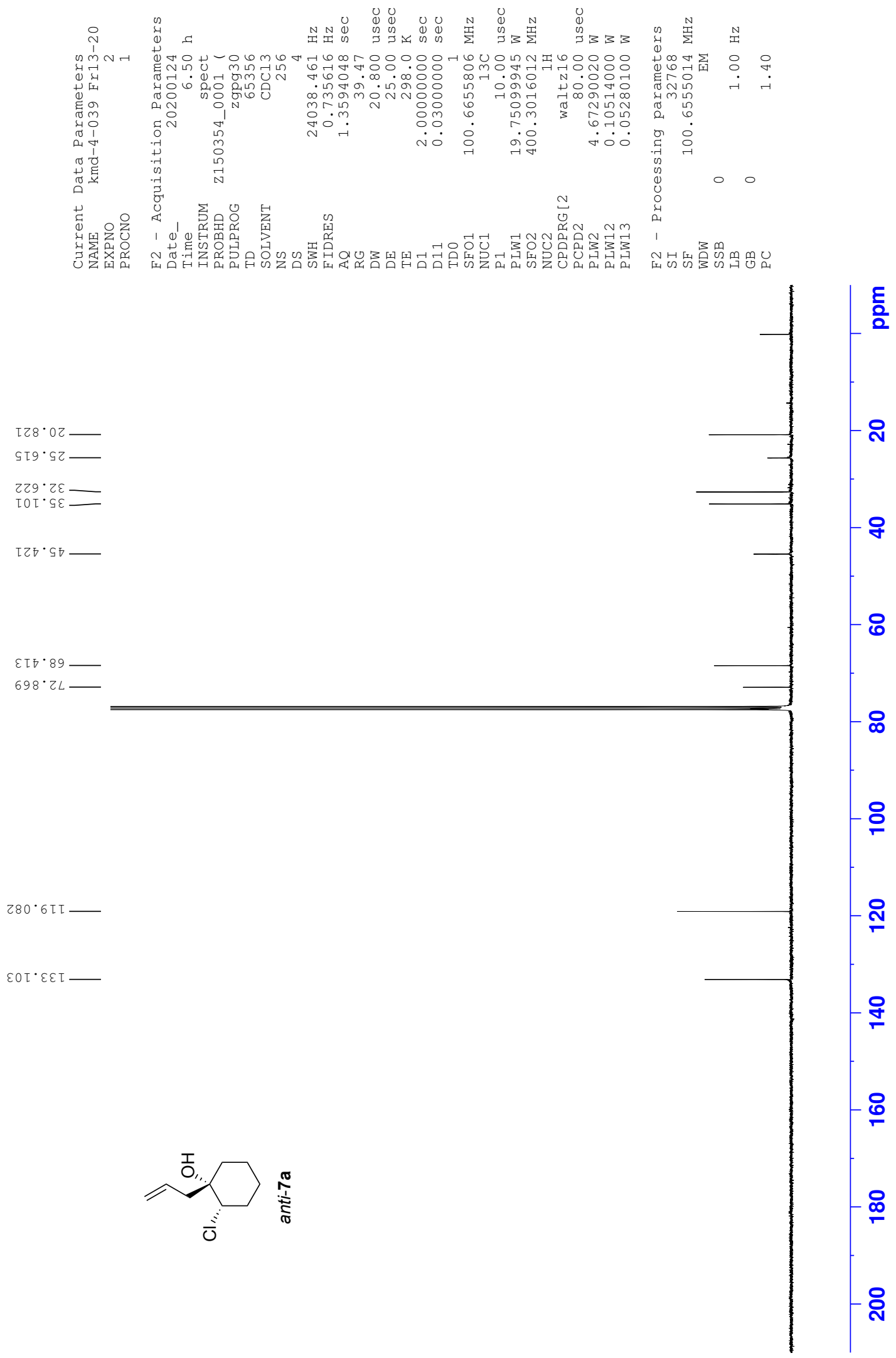

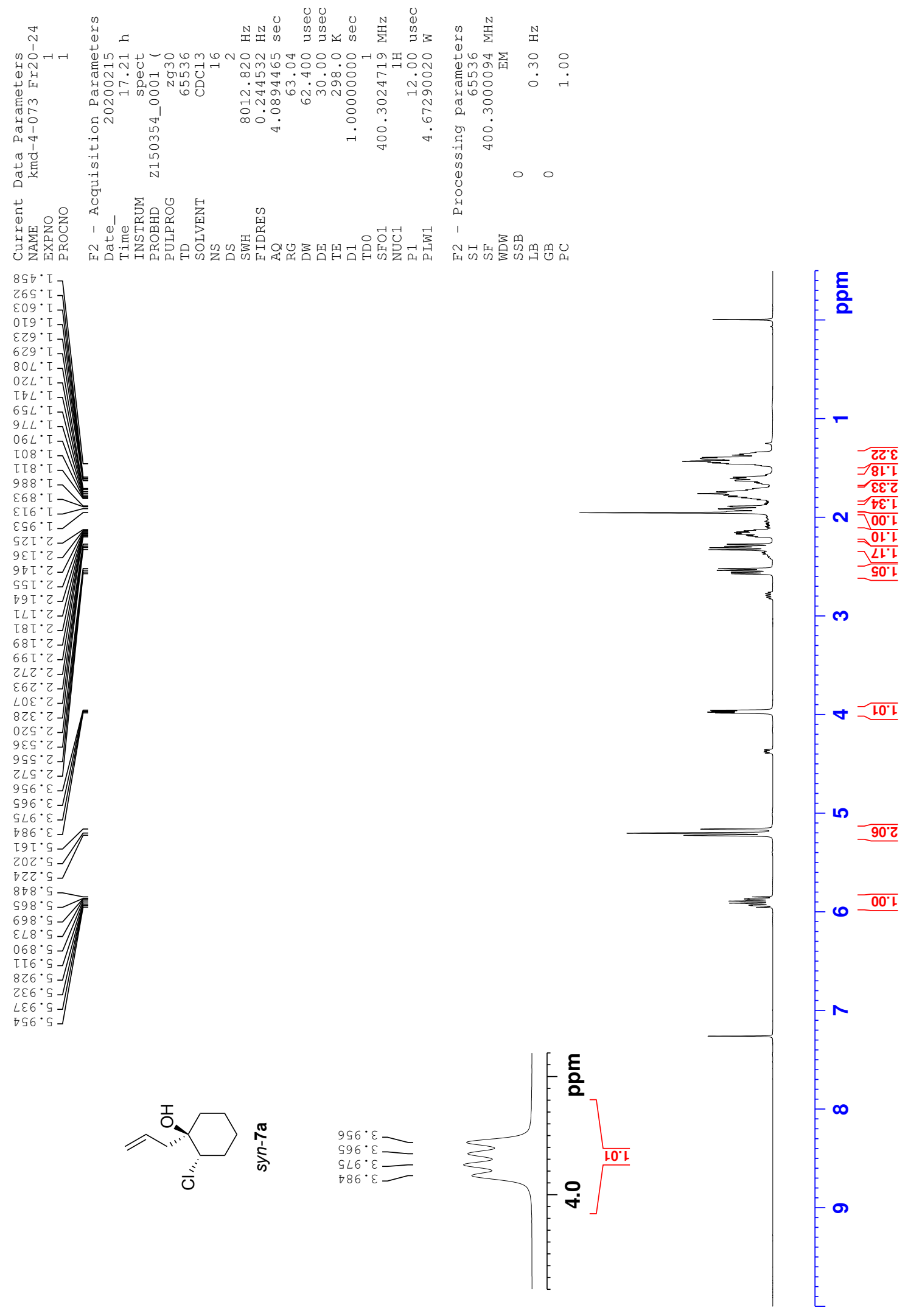

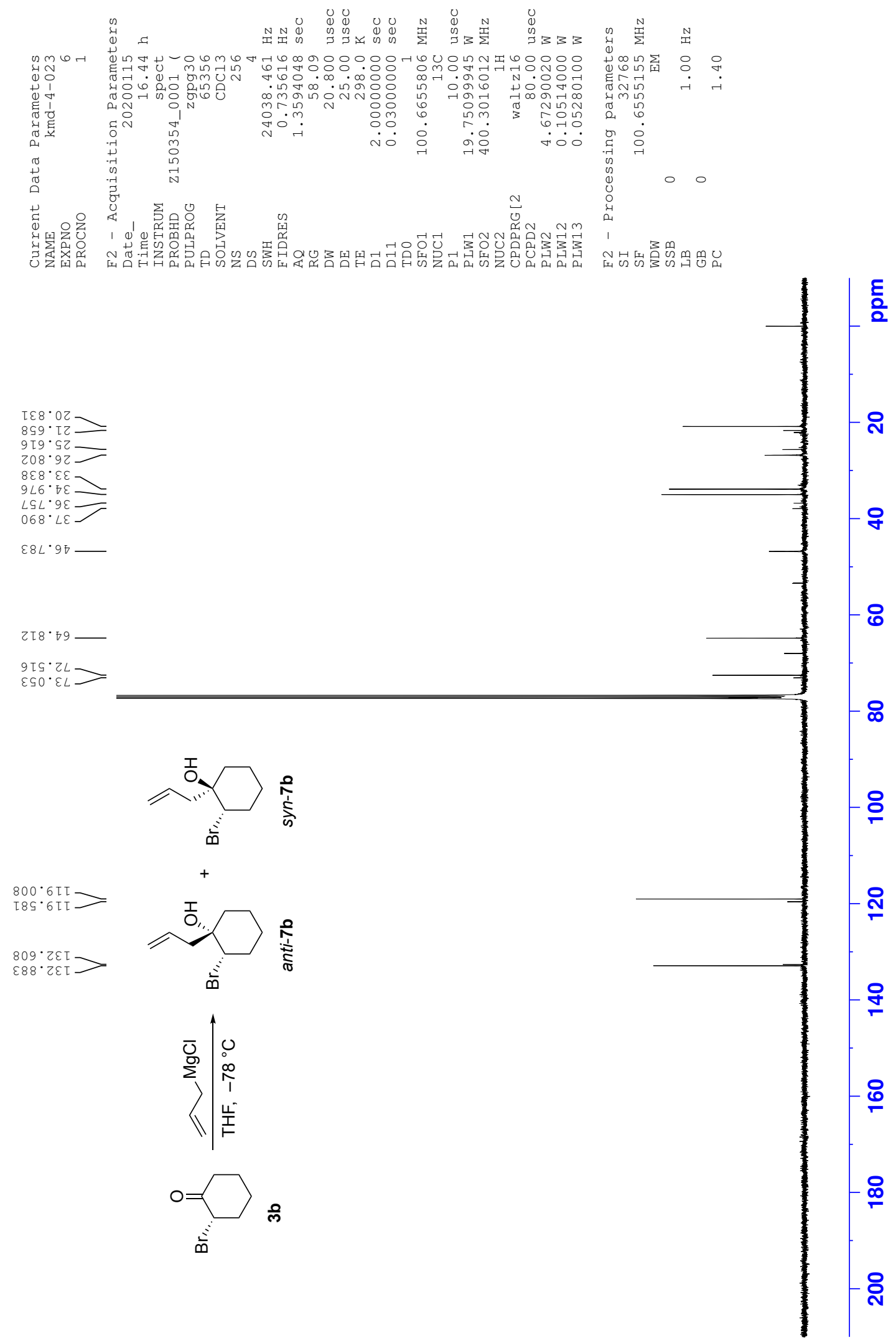


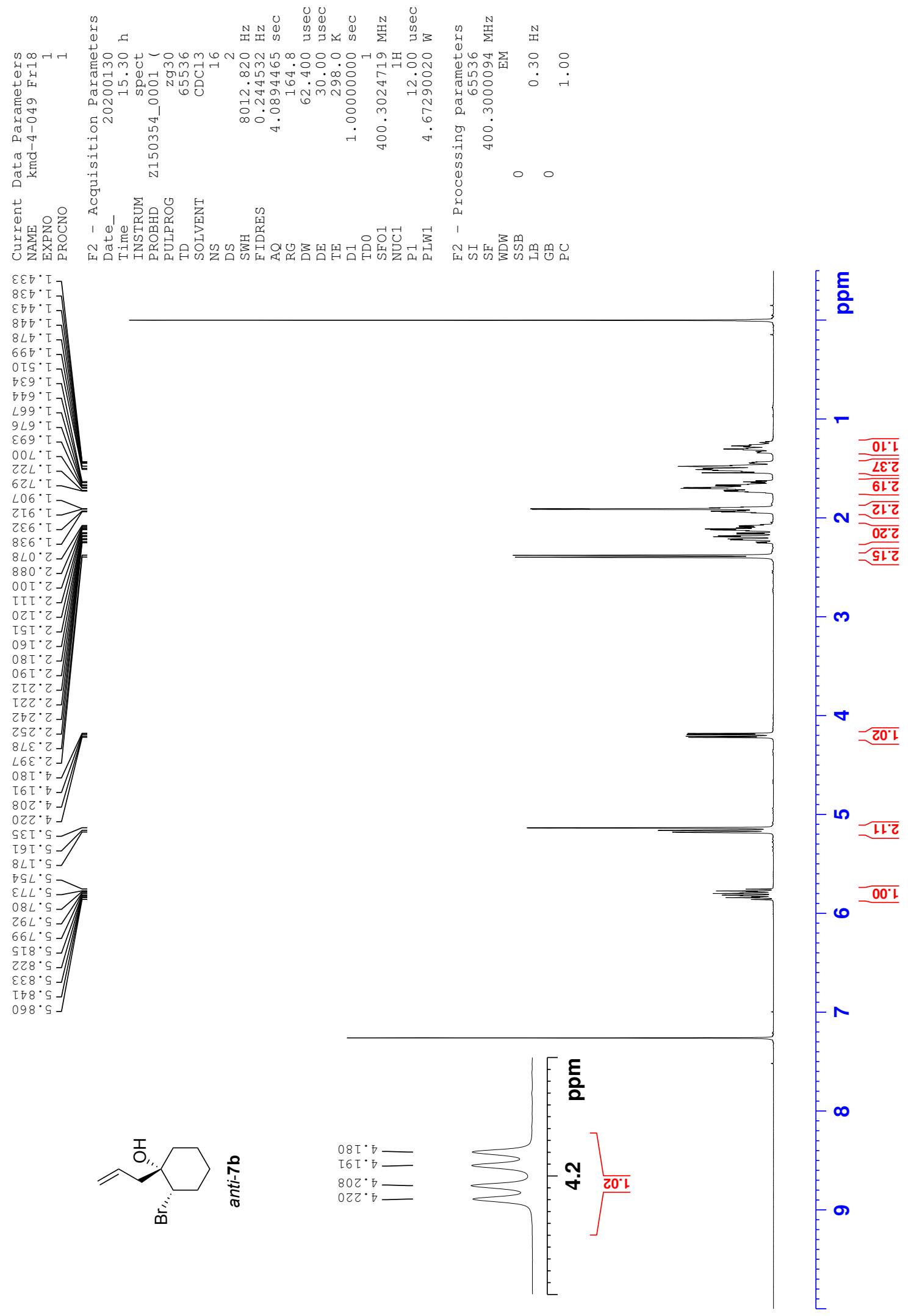



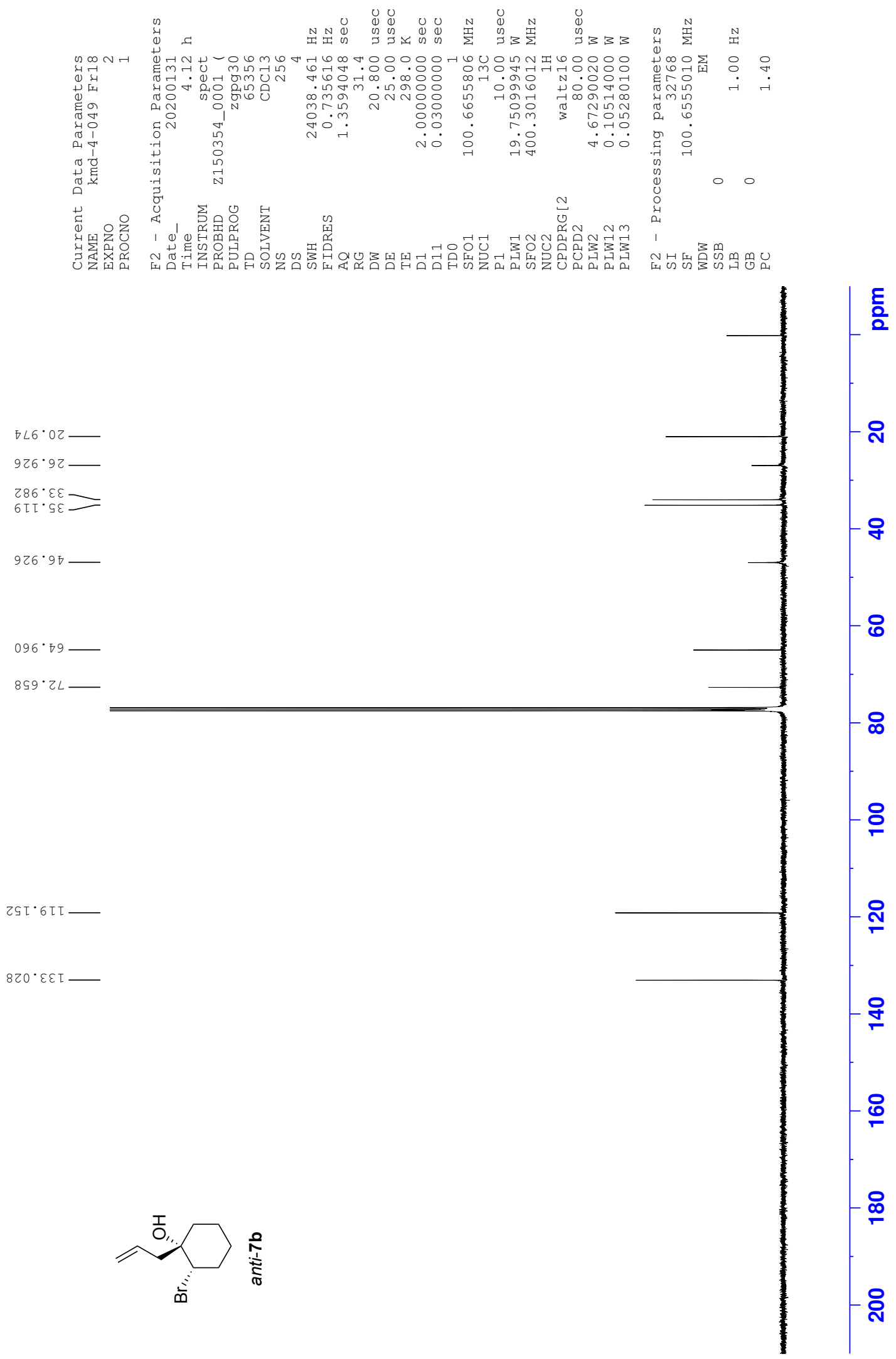

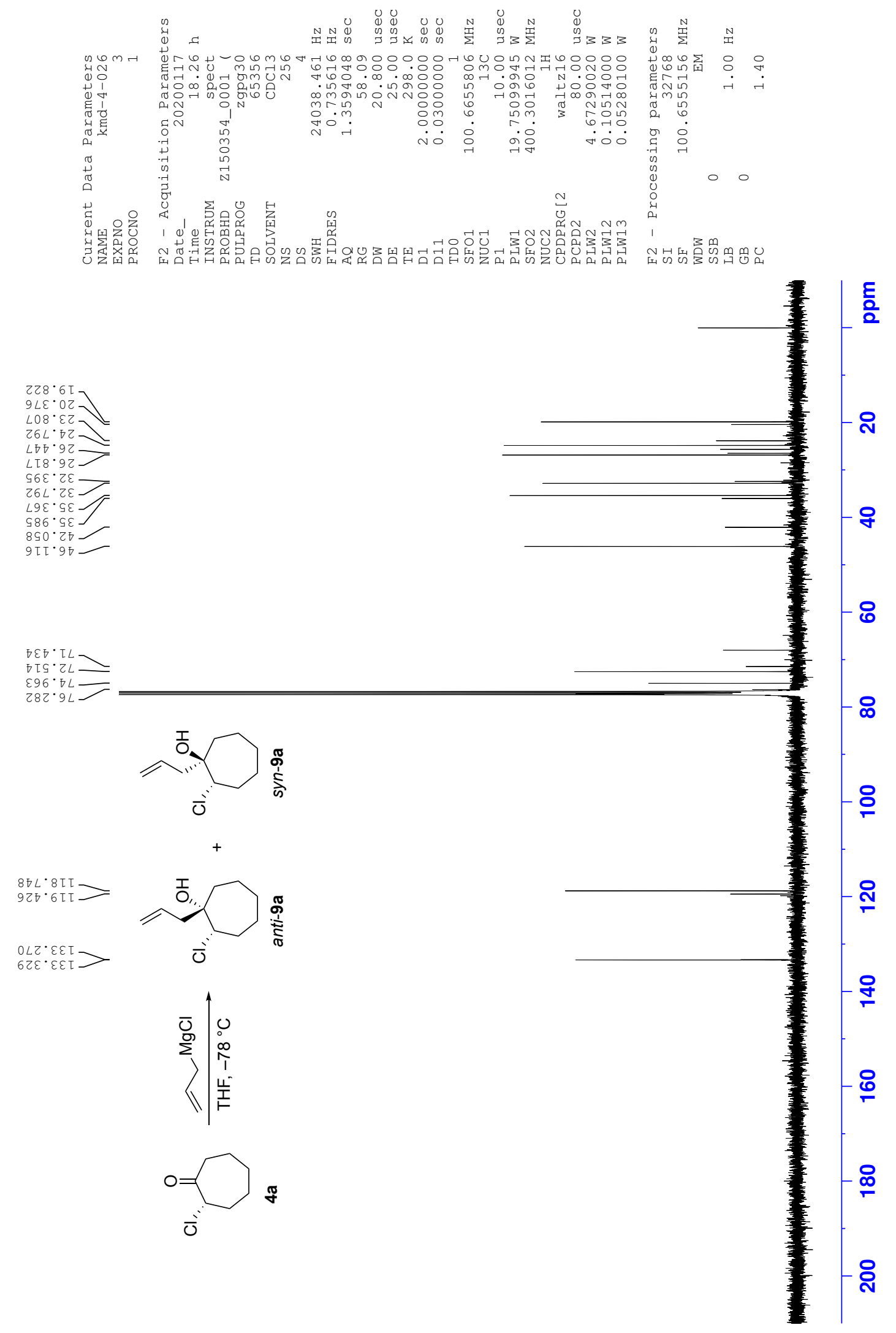

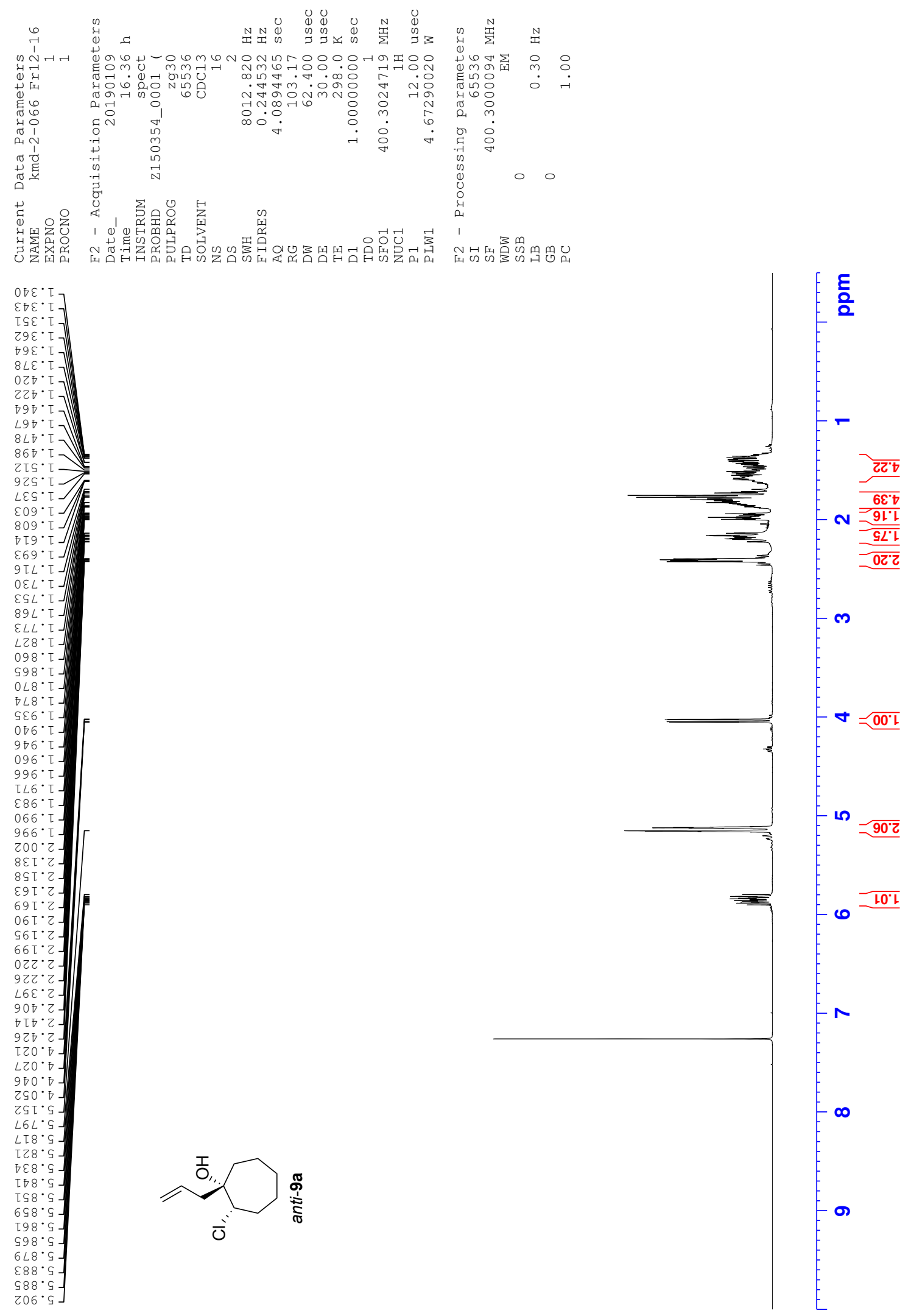

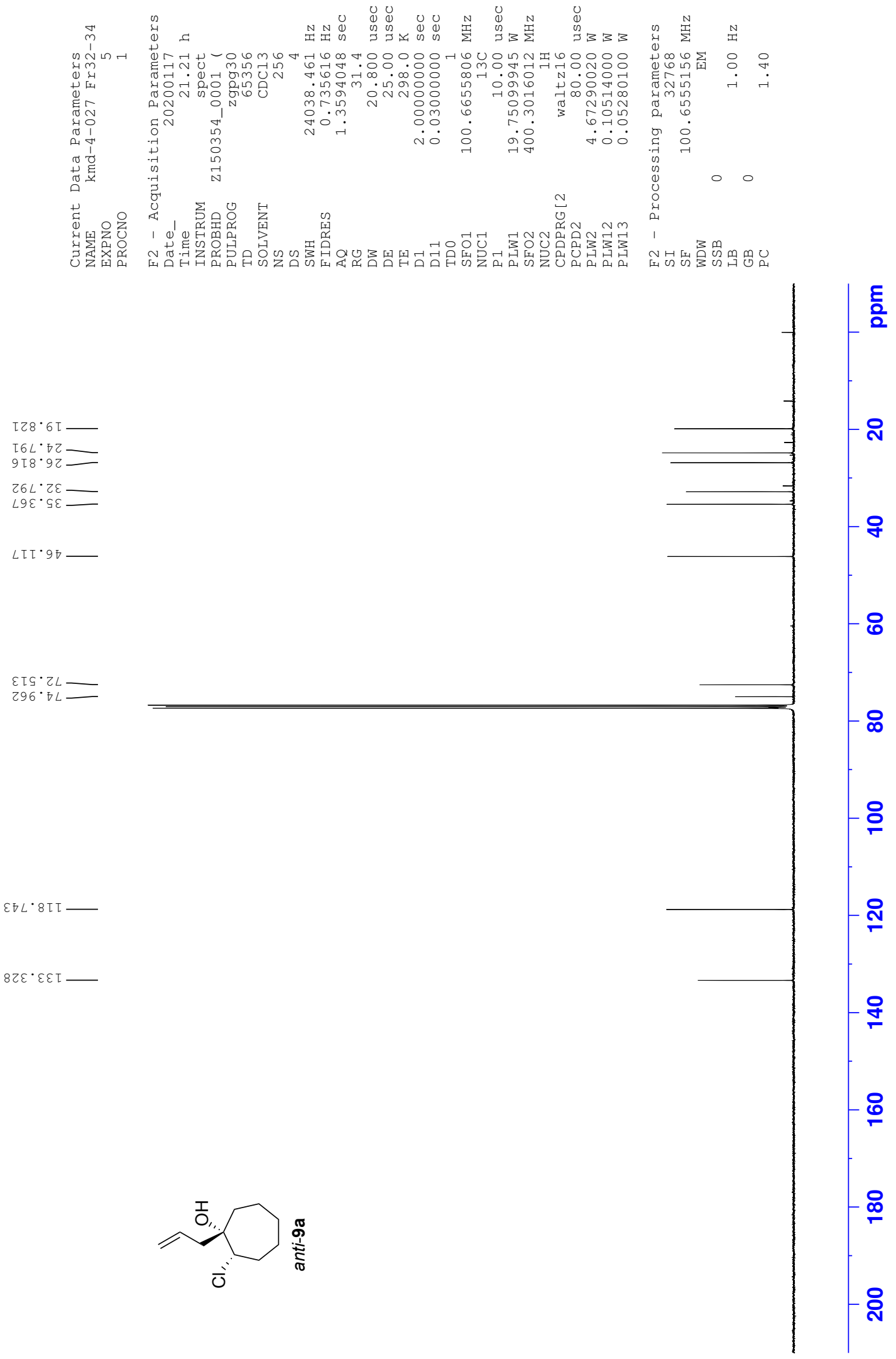

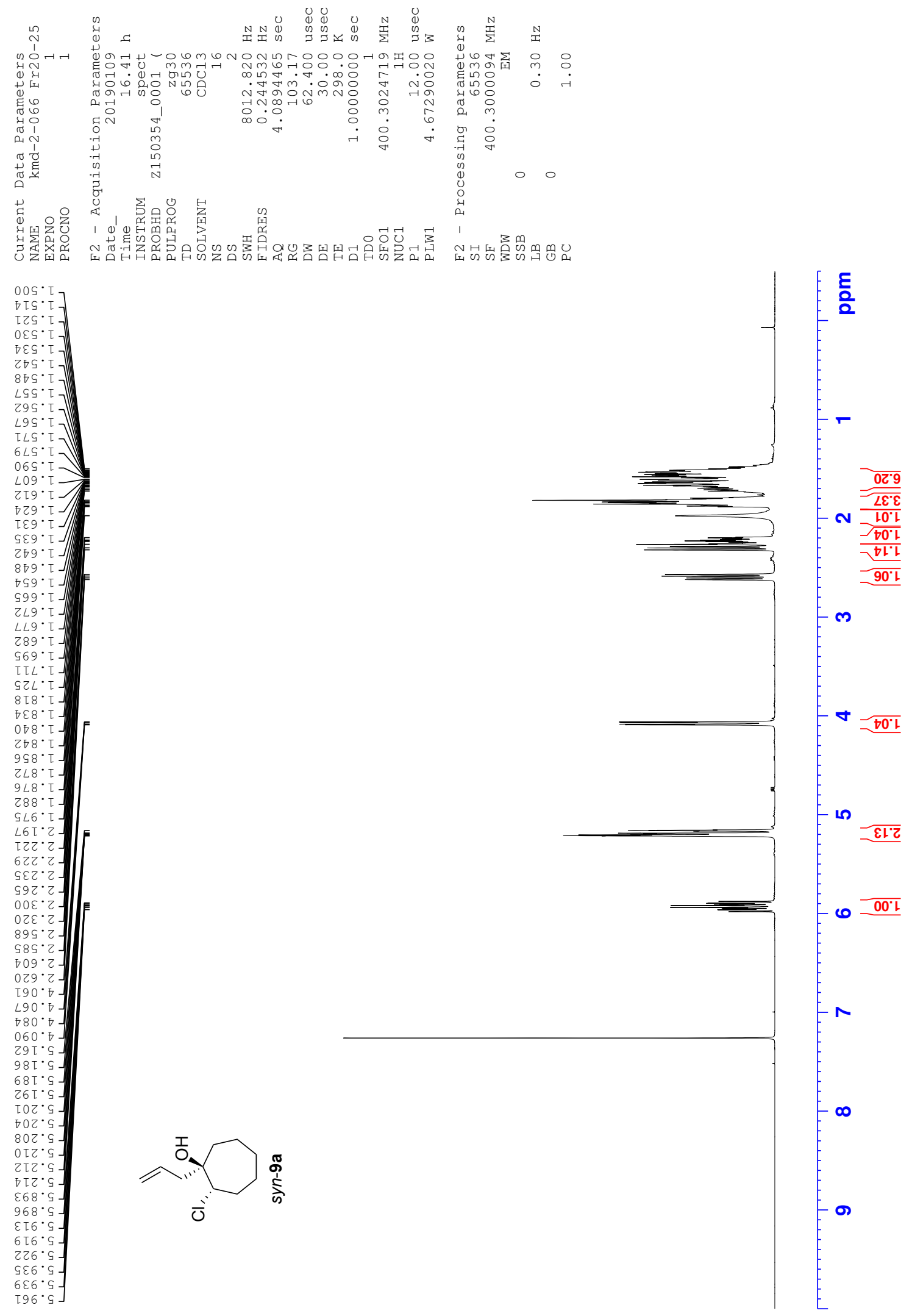

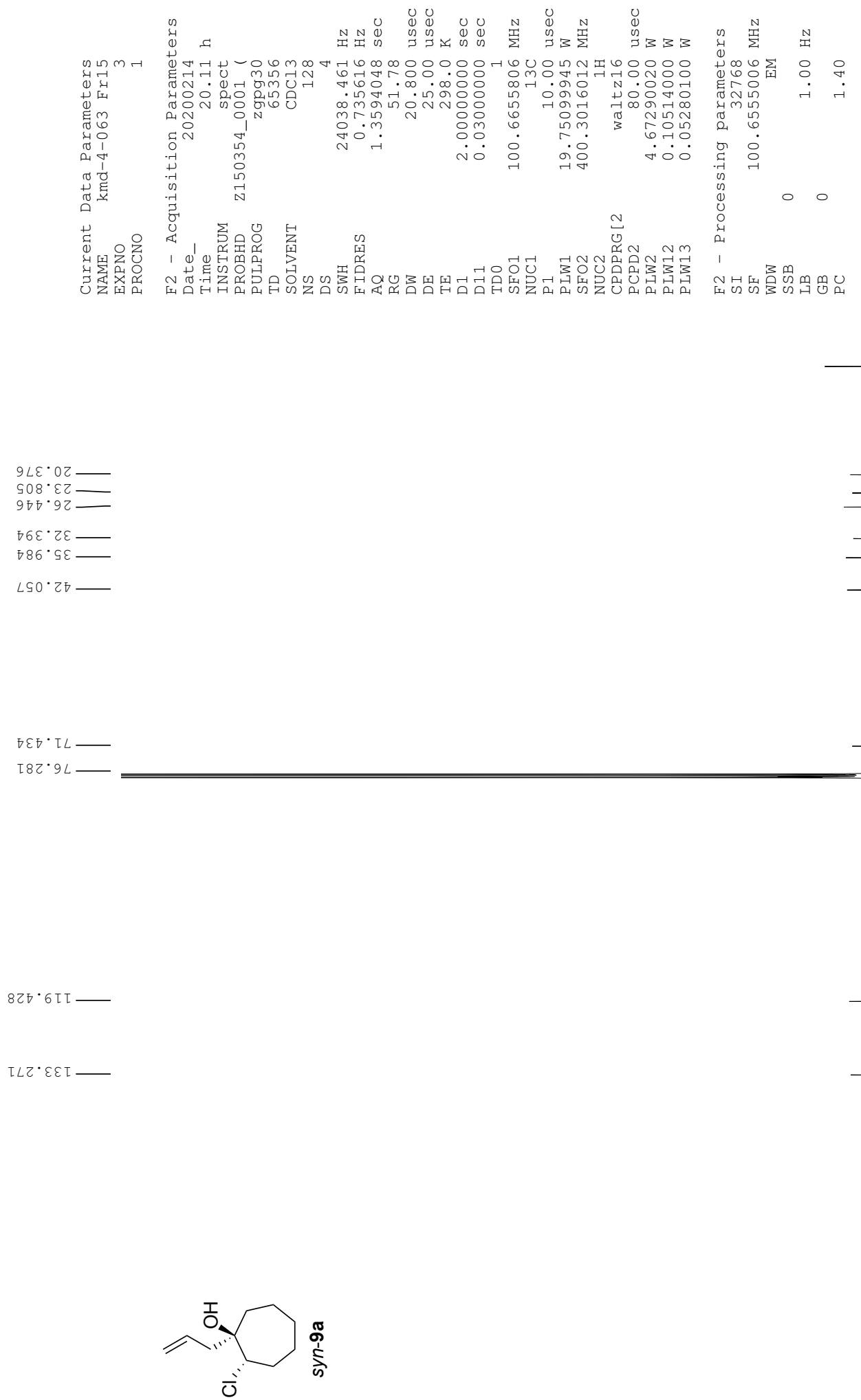

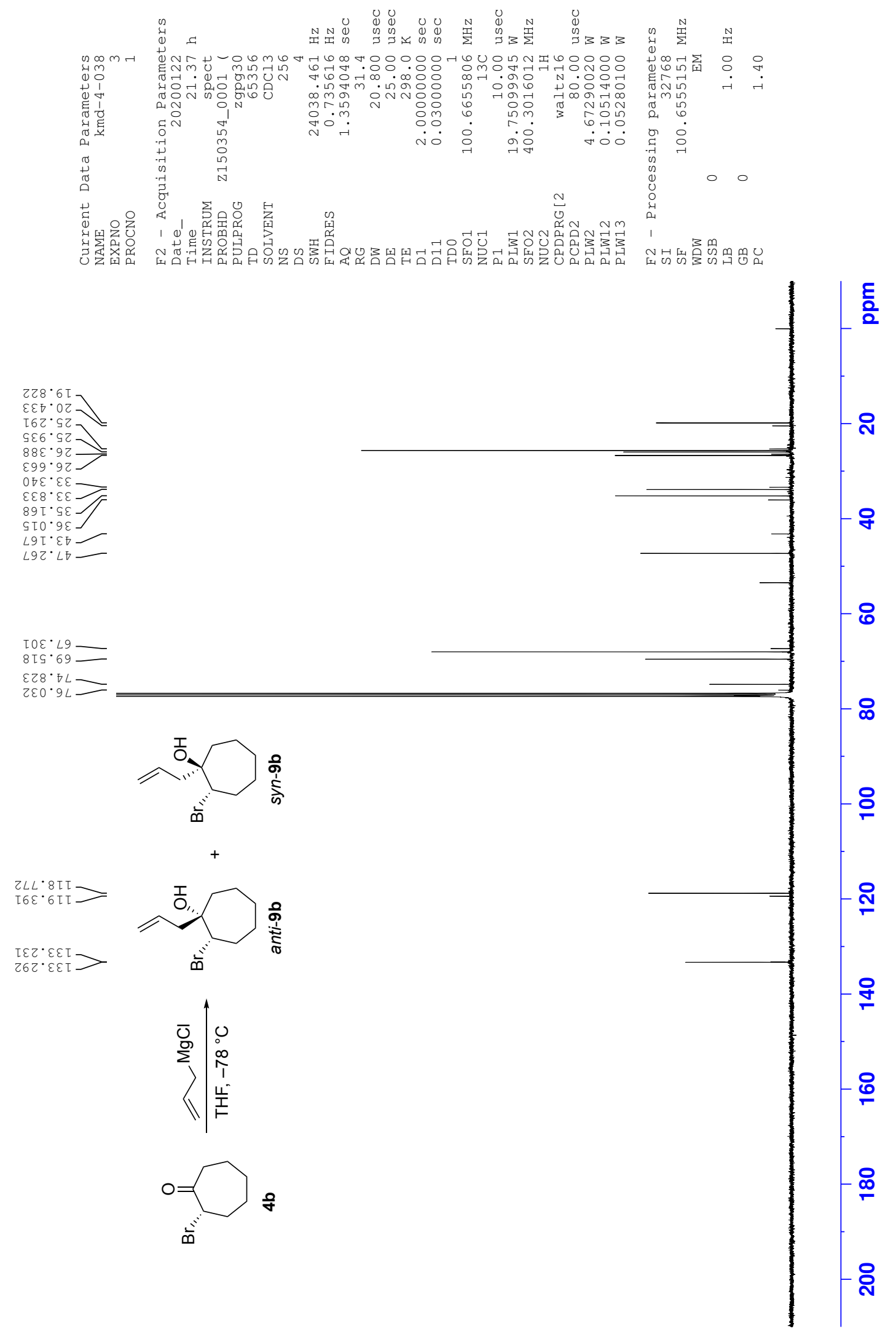

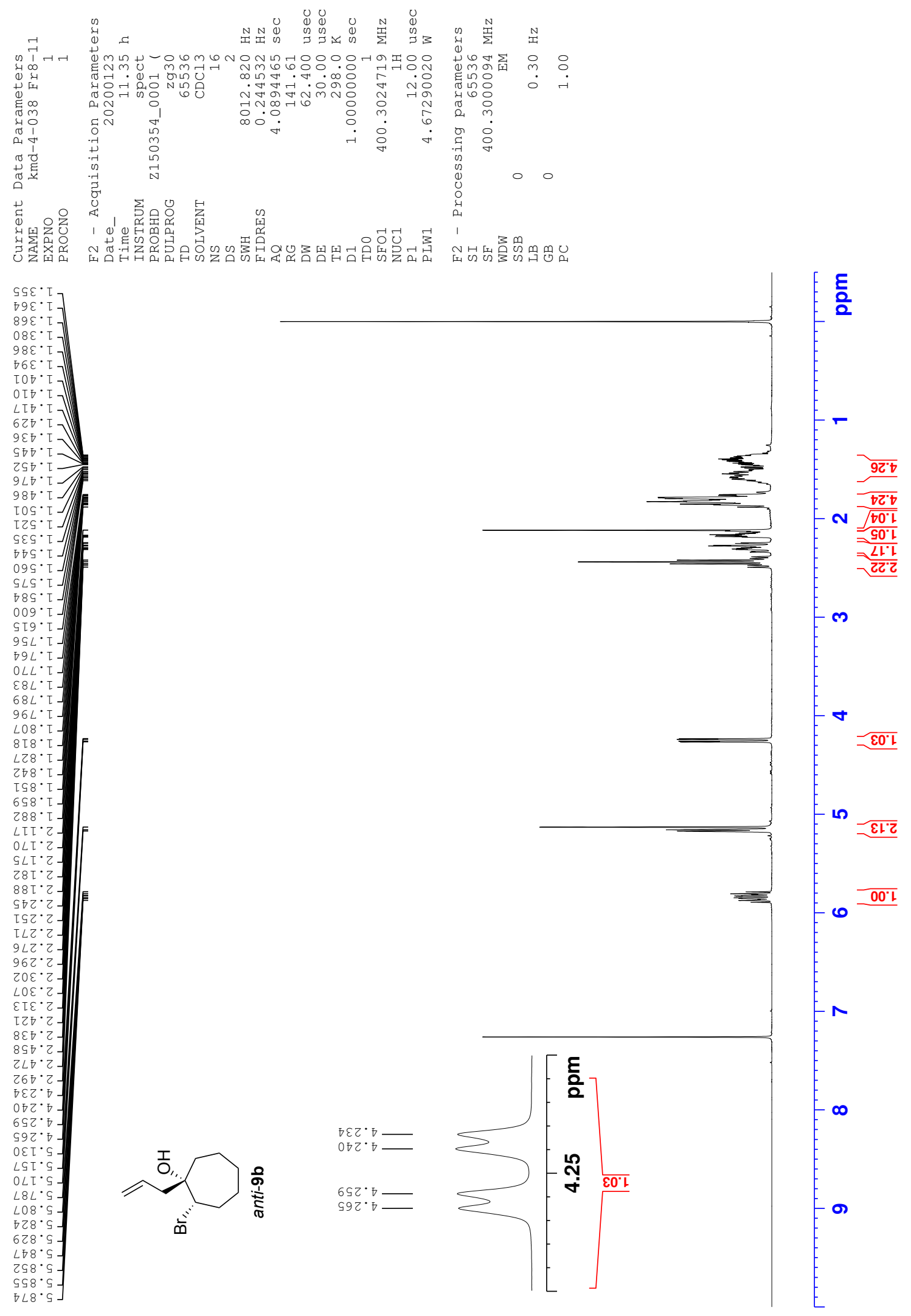

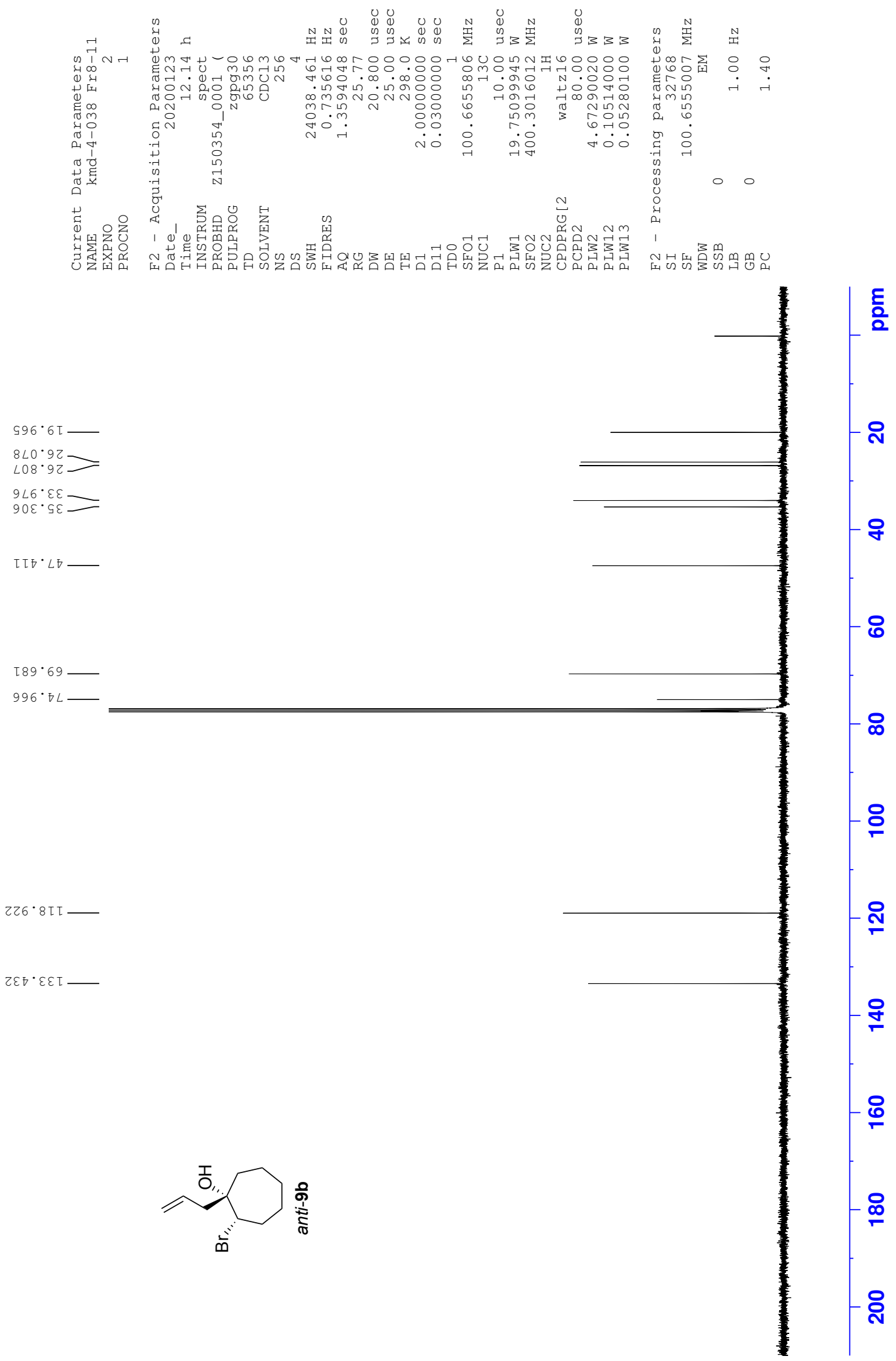

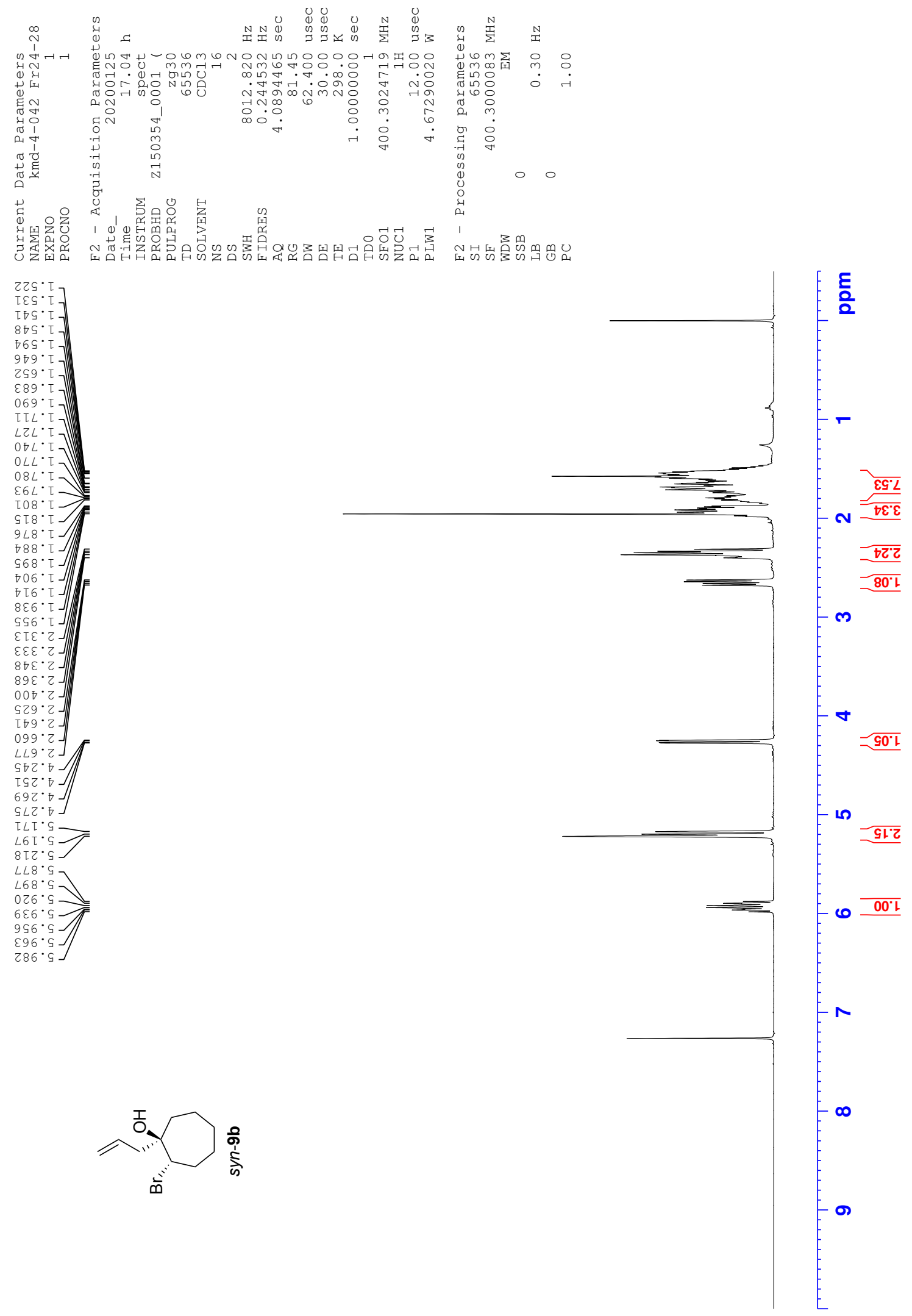

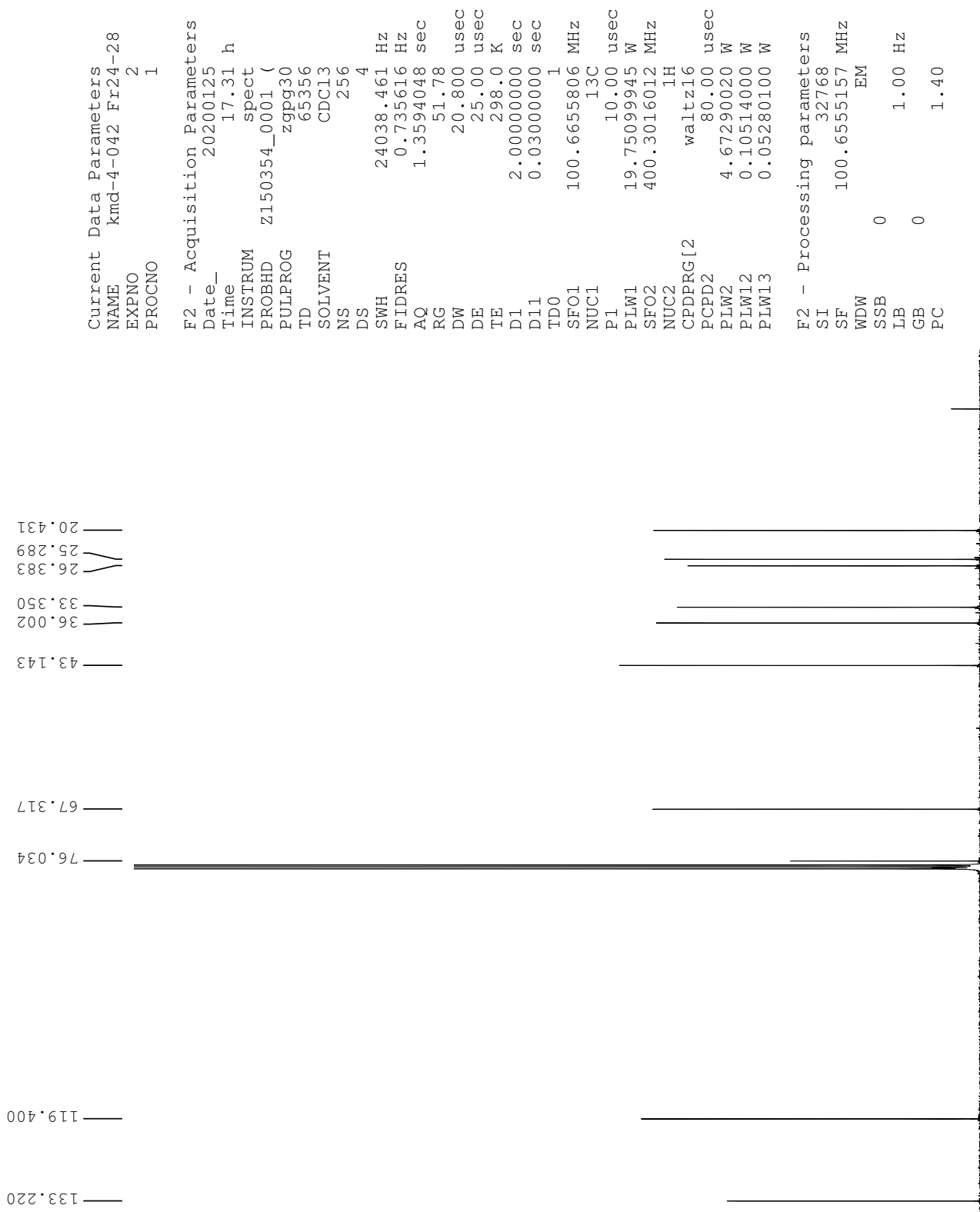

틍

- ำ

우

8

ஃ

우

ํำ

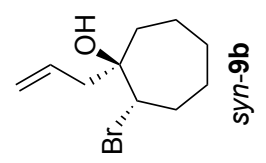

की

$\stackrel{\infty}{-}$

$\underline{8}$

ঃั 

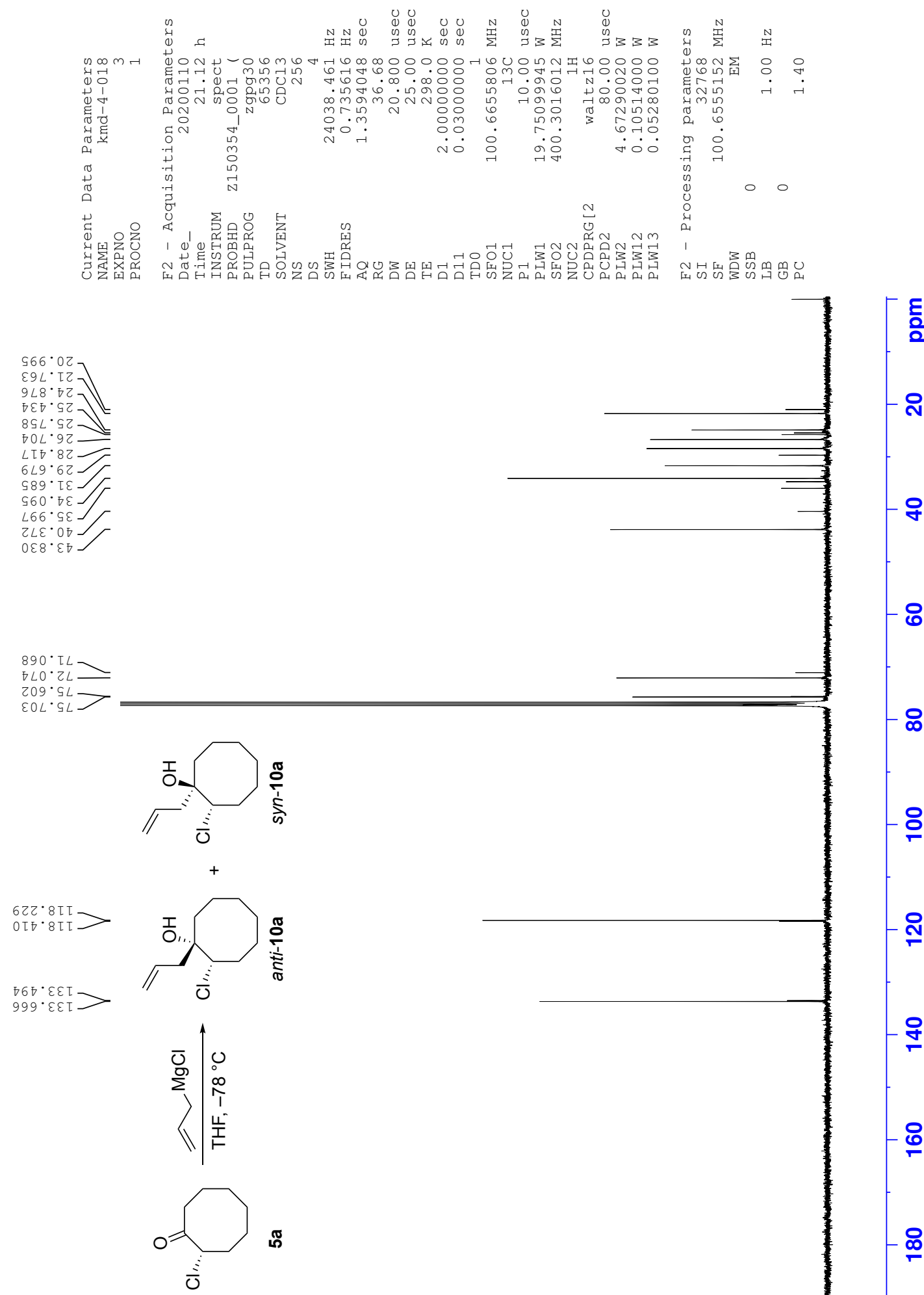


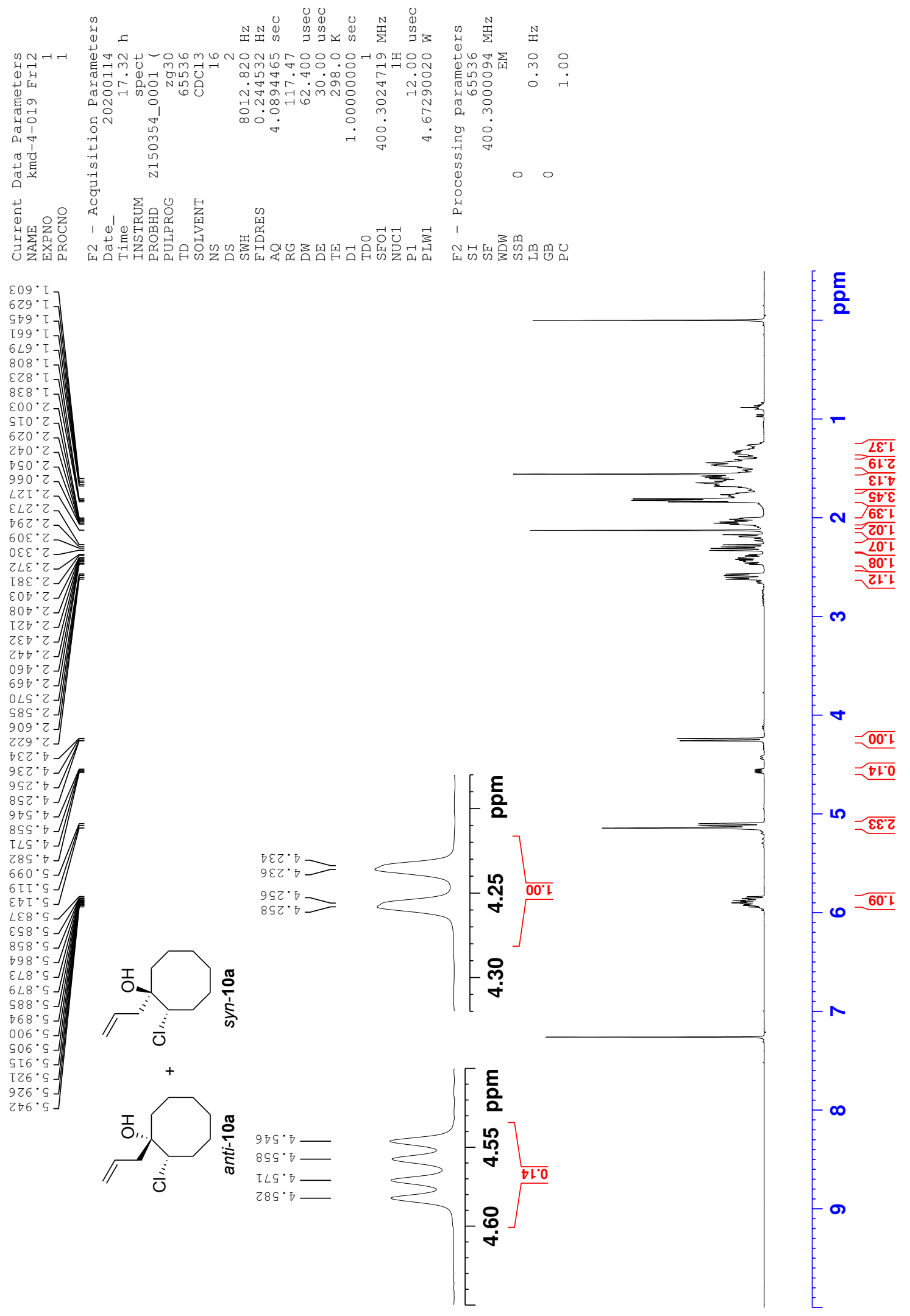



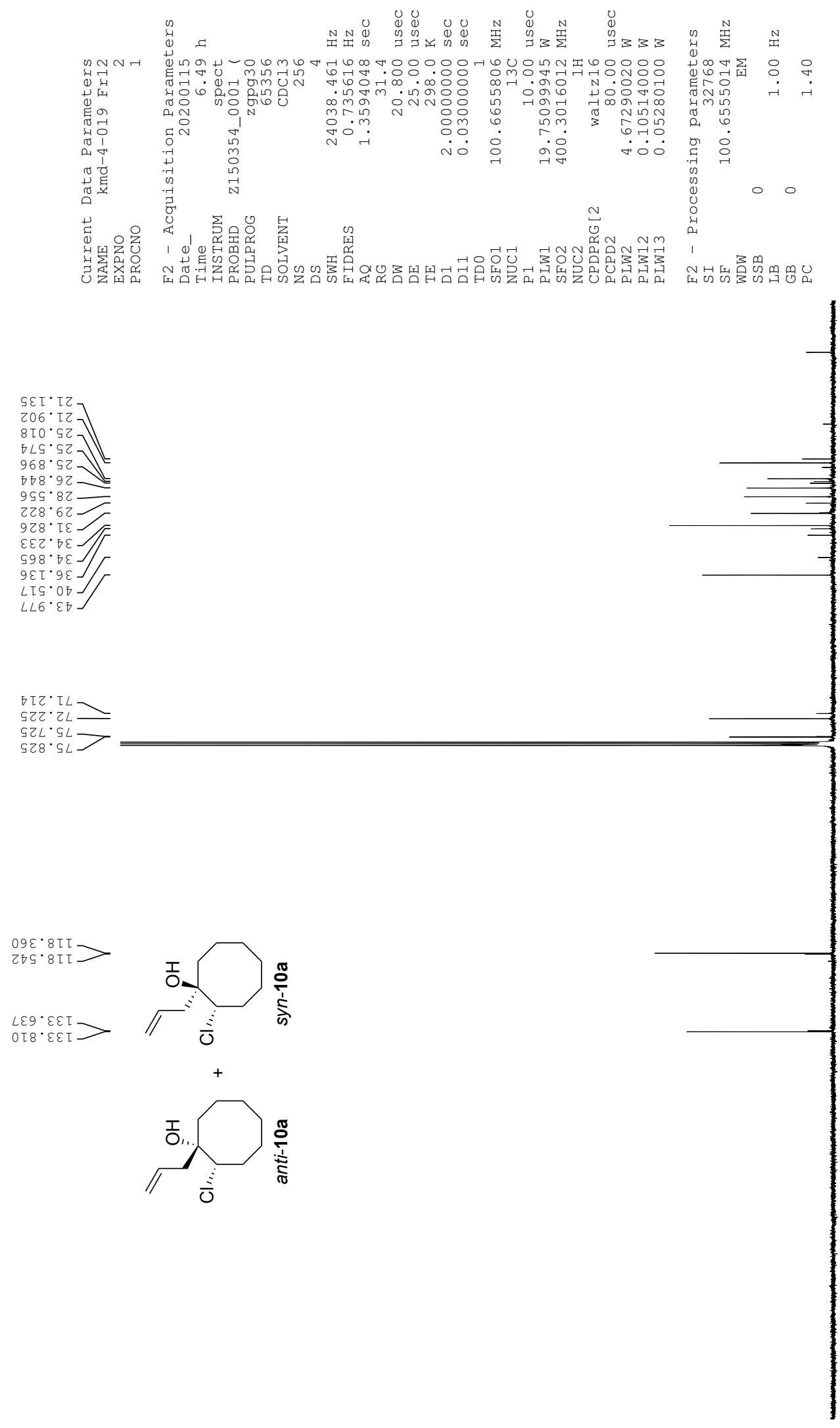

-

은

ิㅗ

옴

음

ळ

จํ 

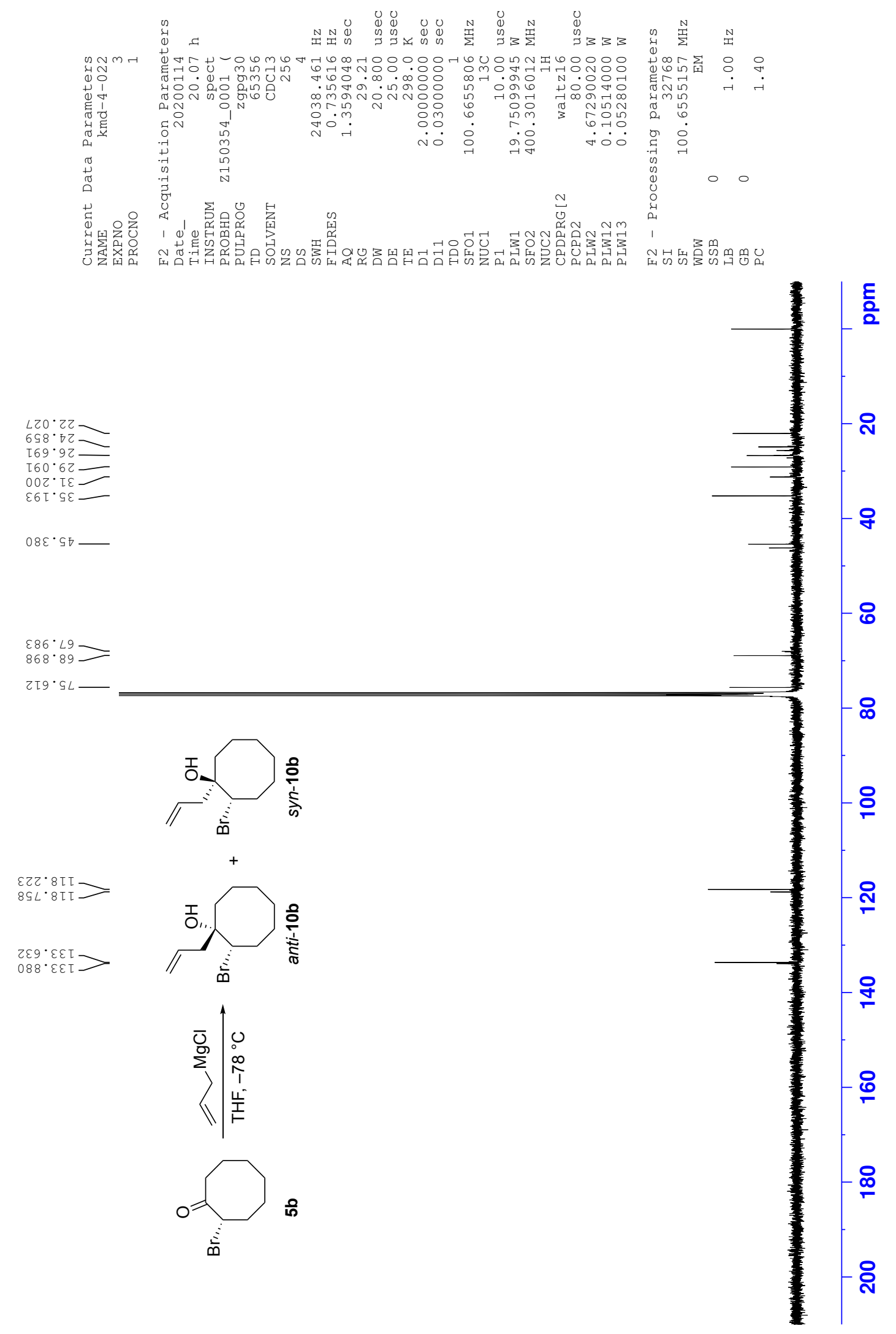


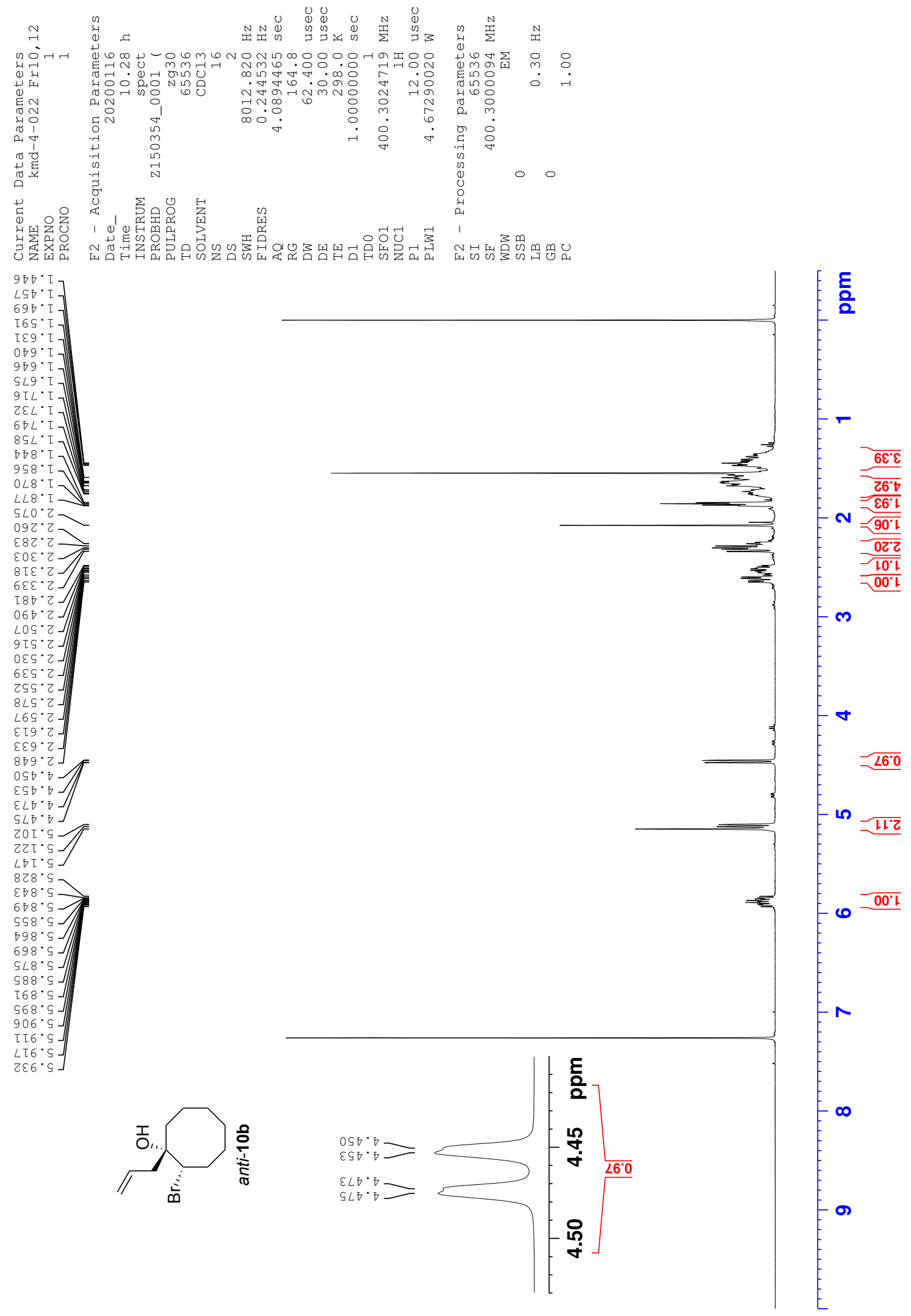



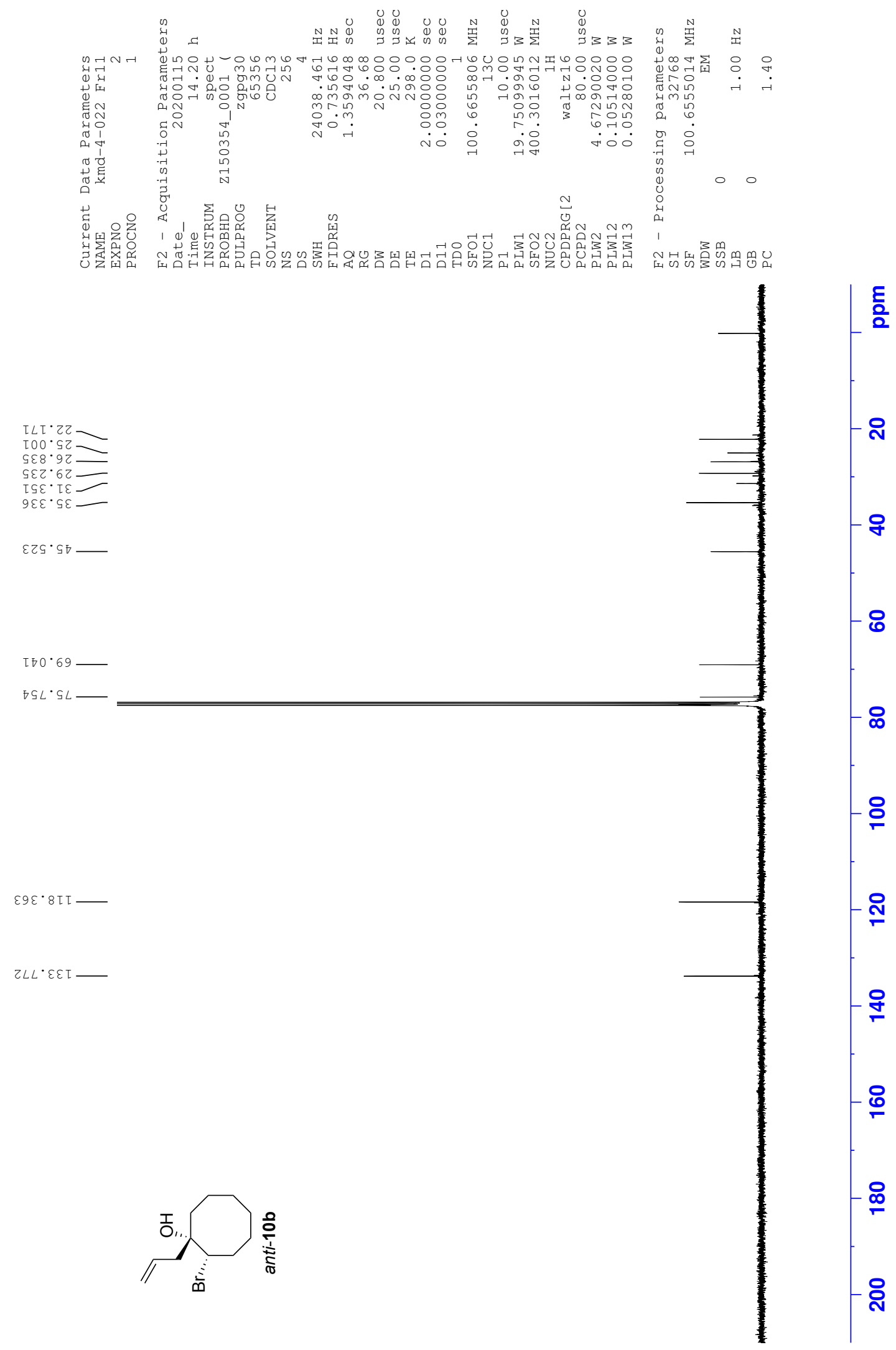

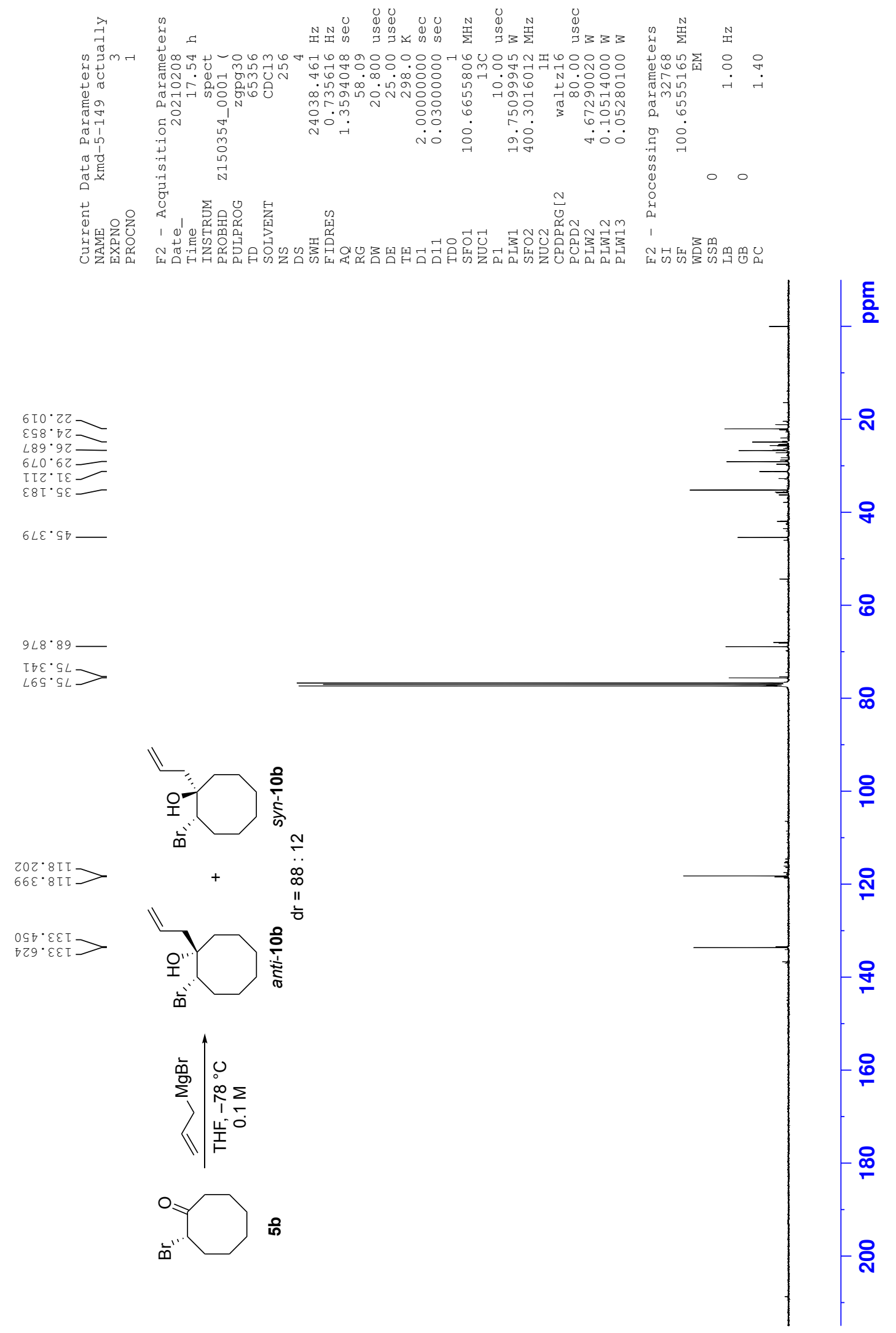

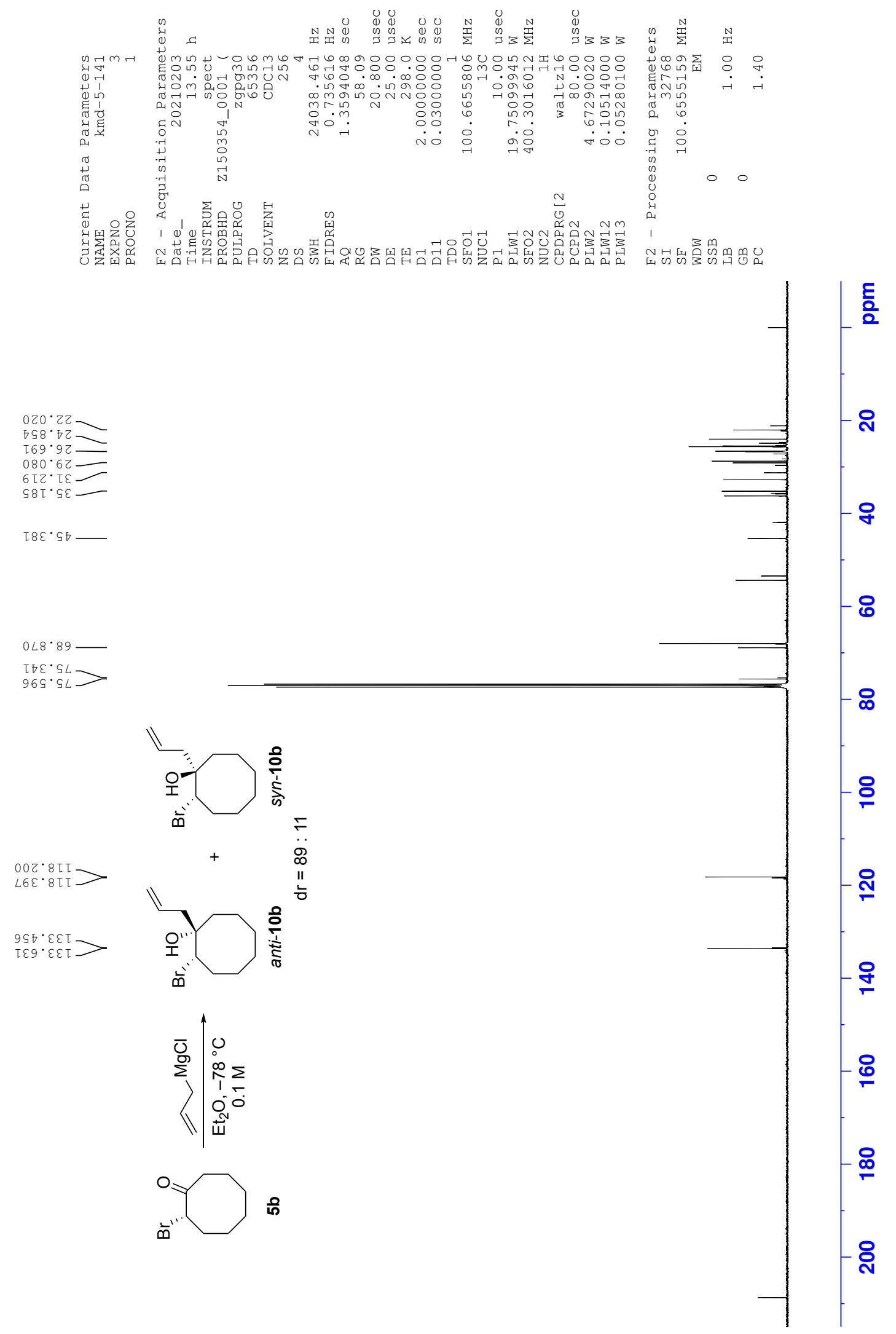

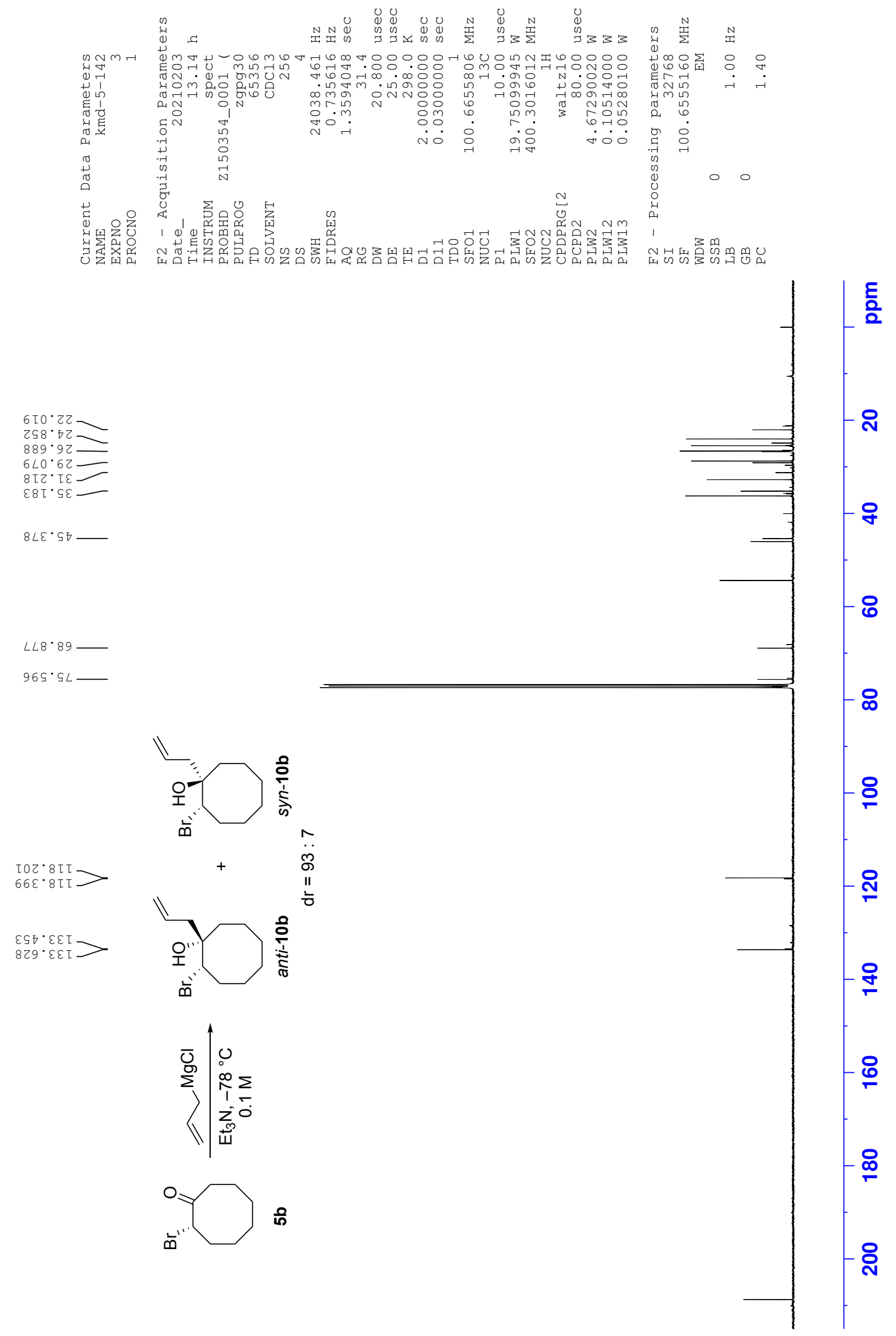

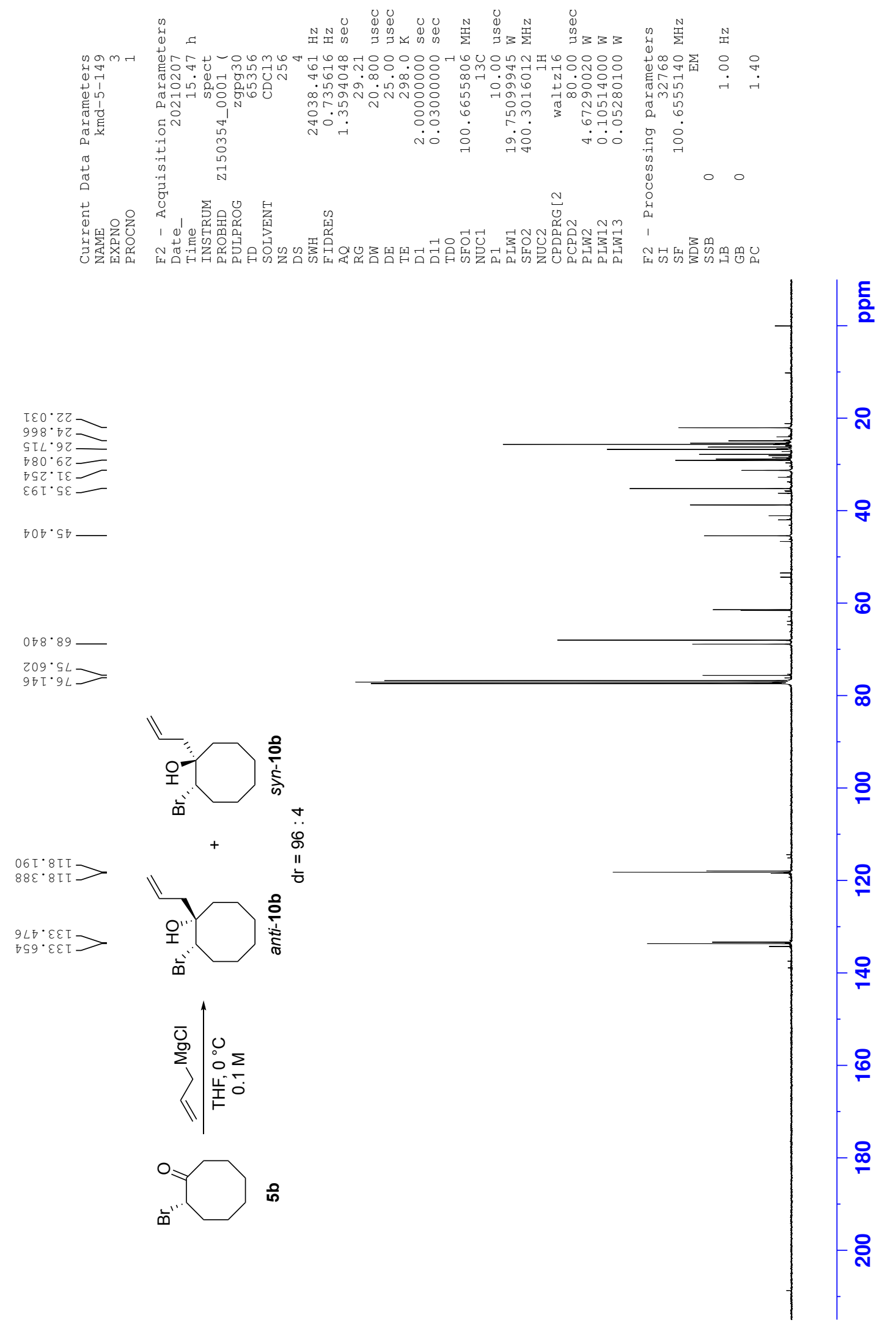

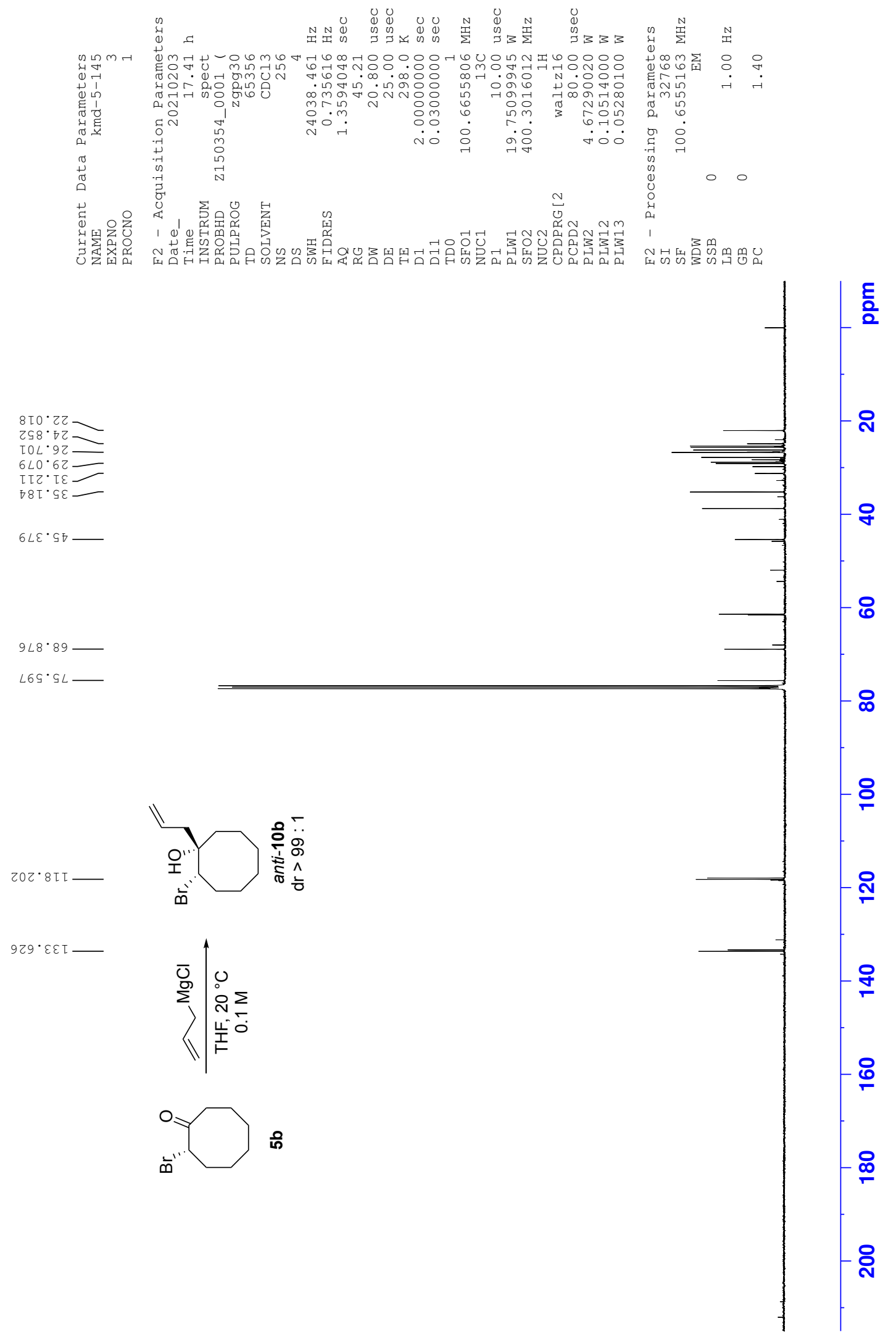

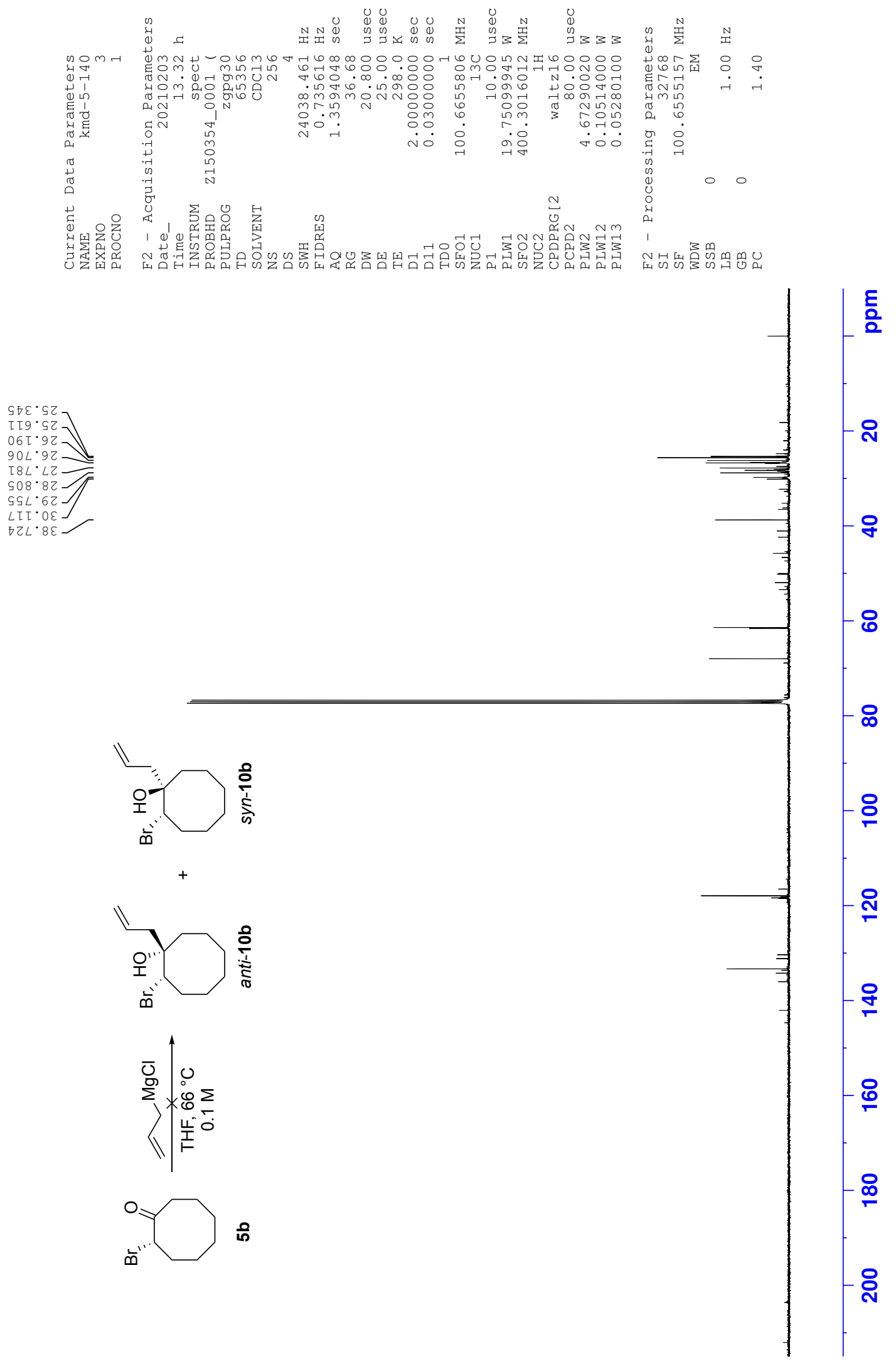

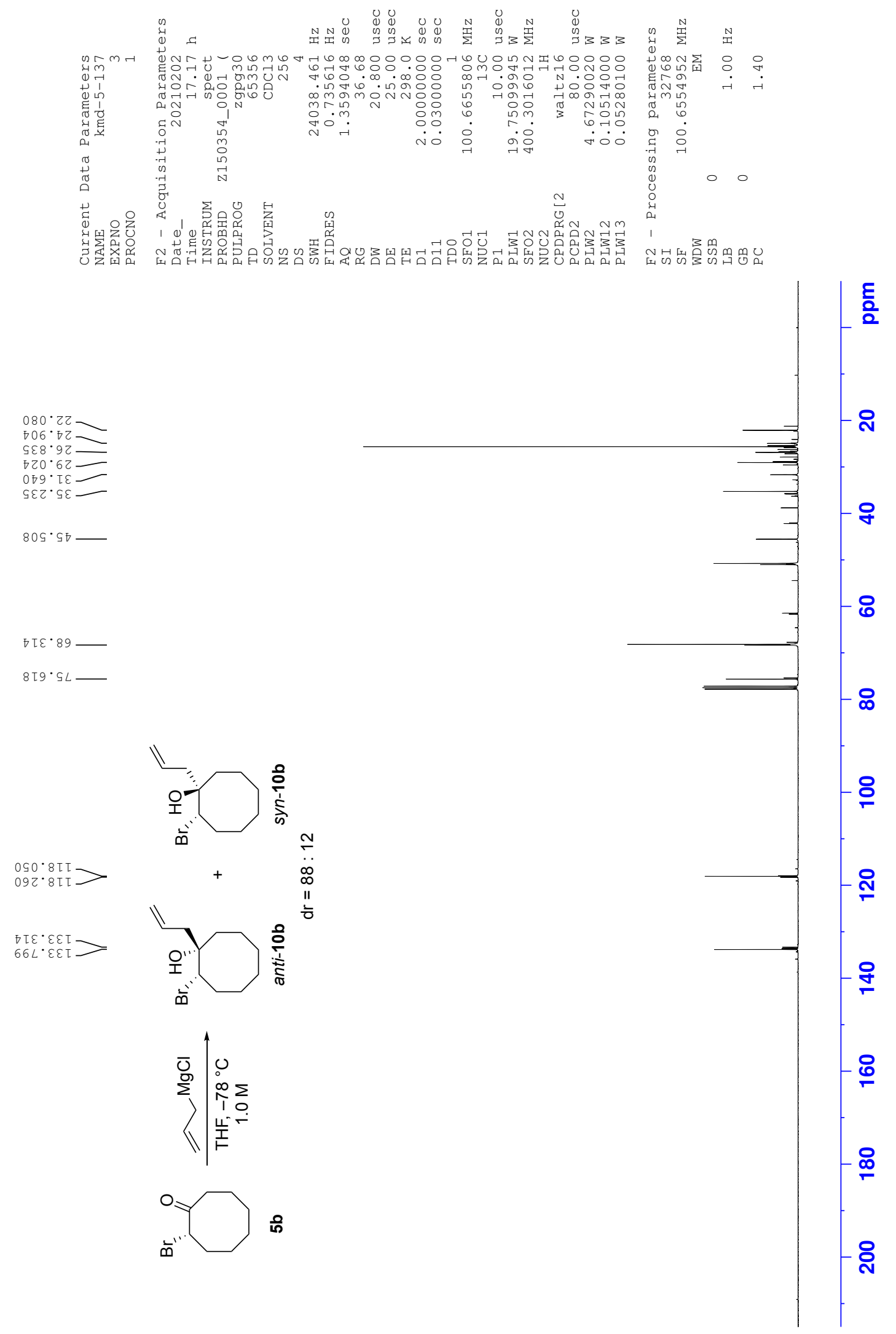

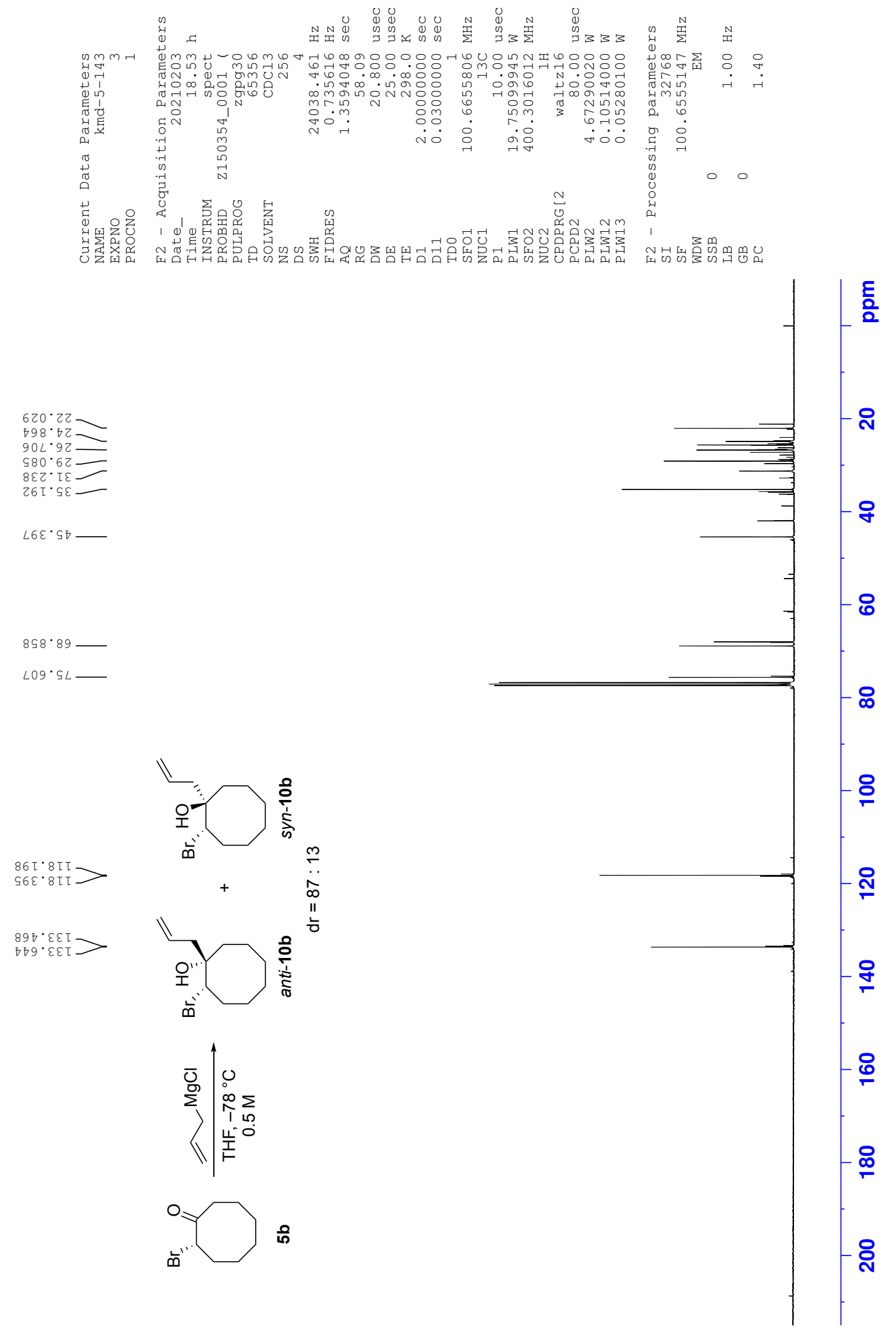

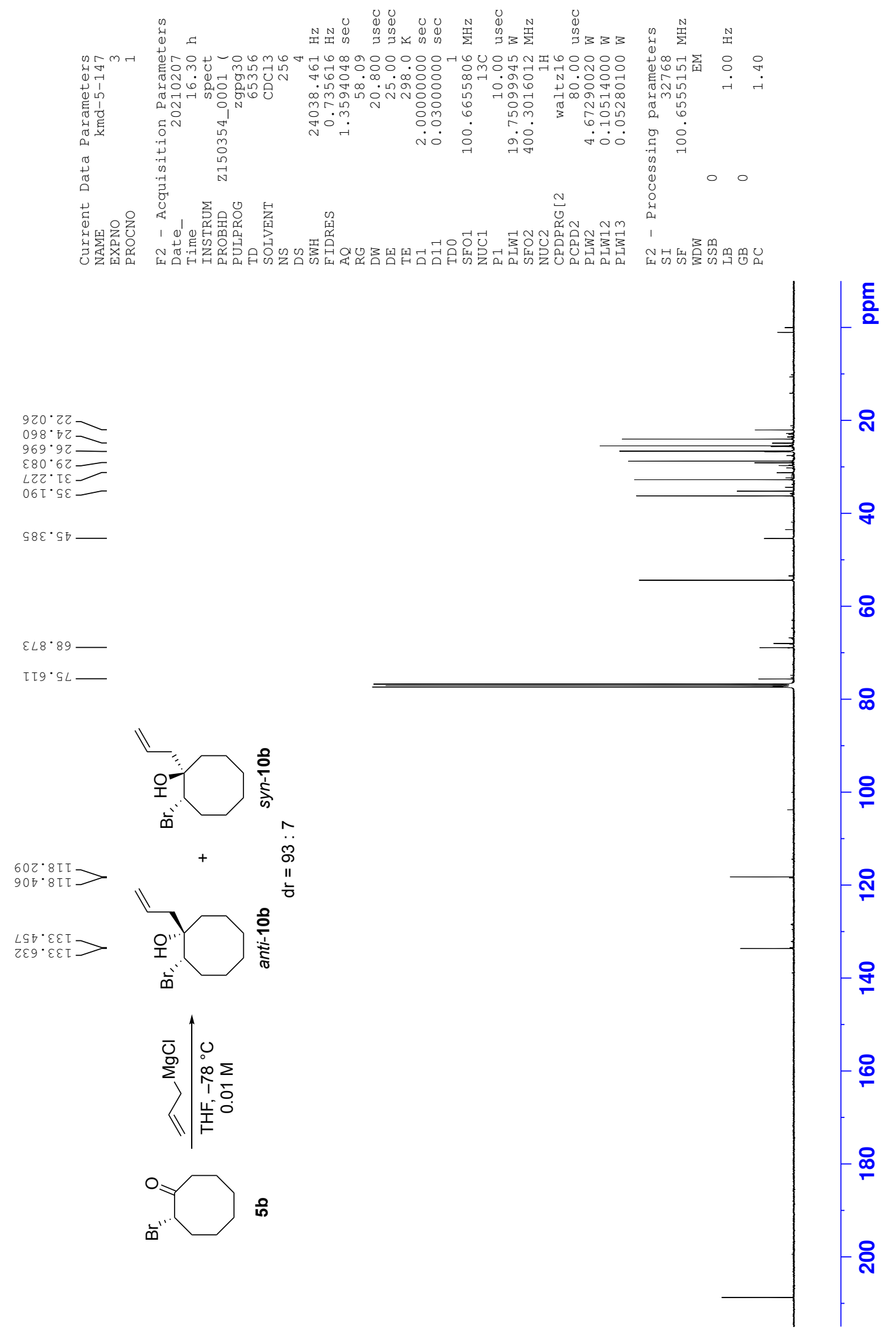

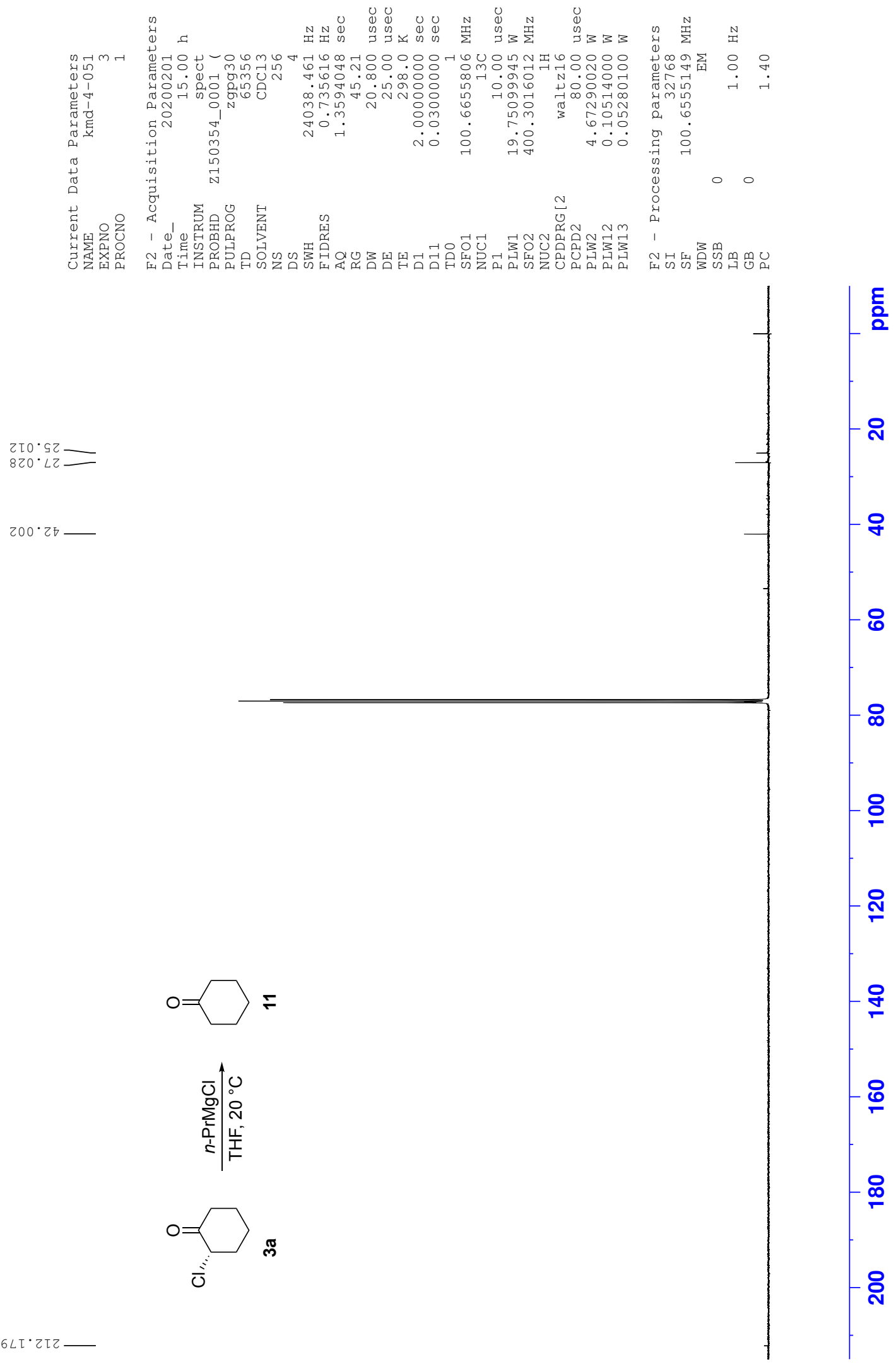

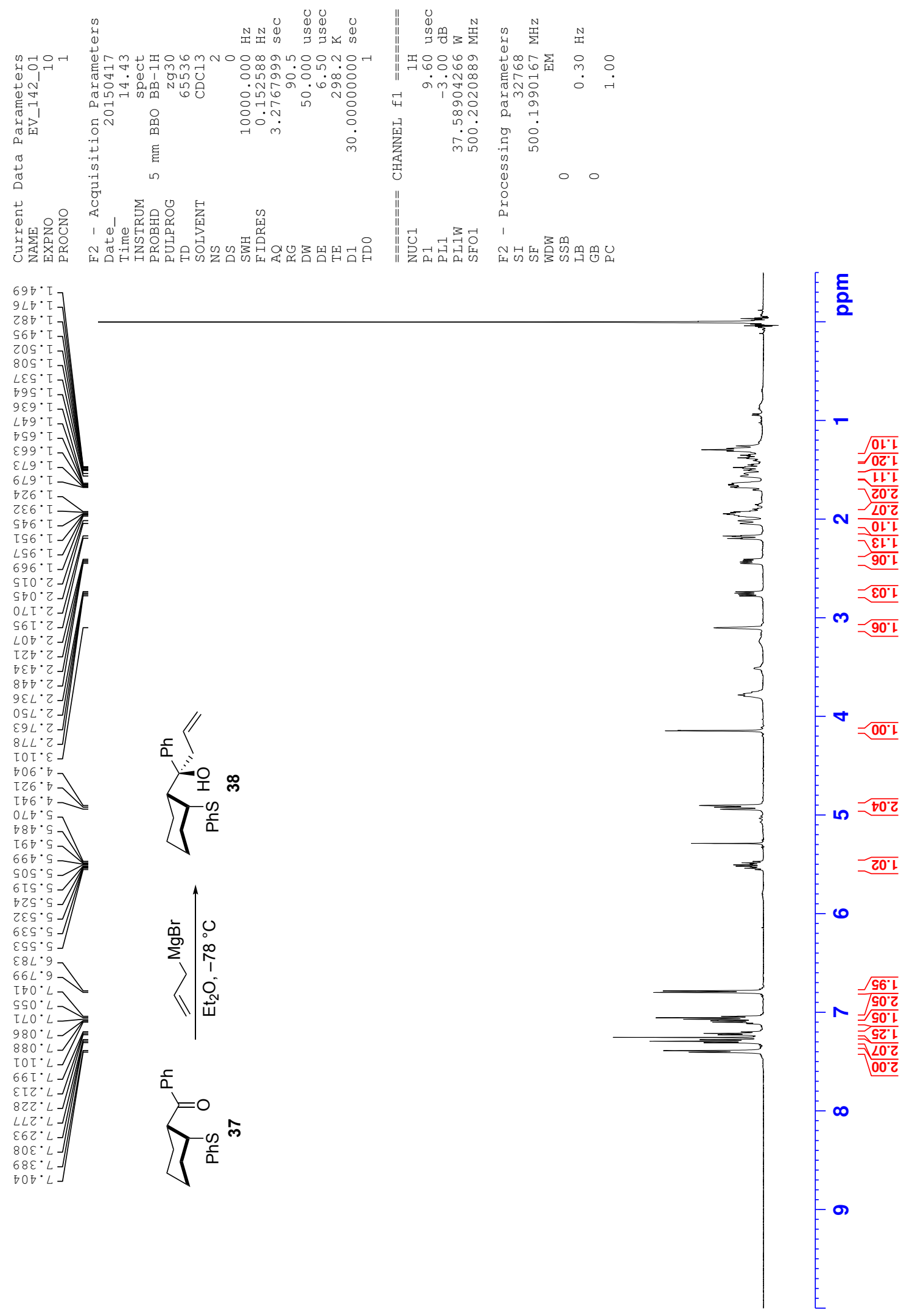

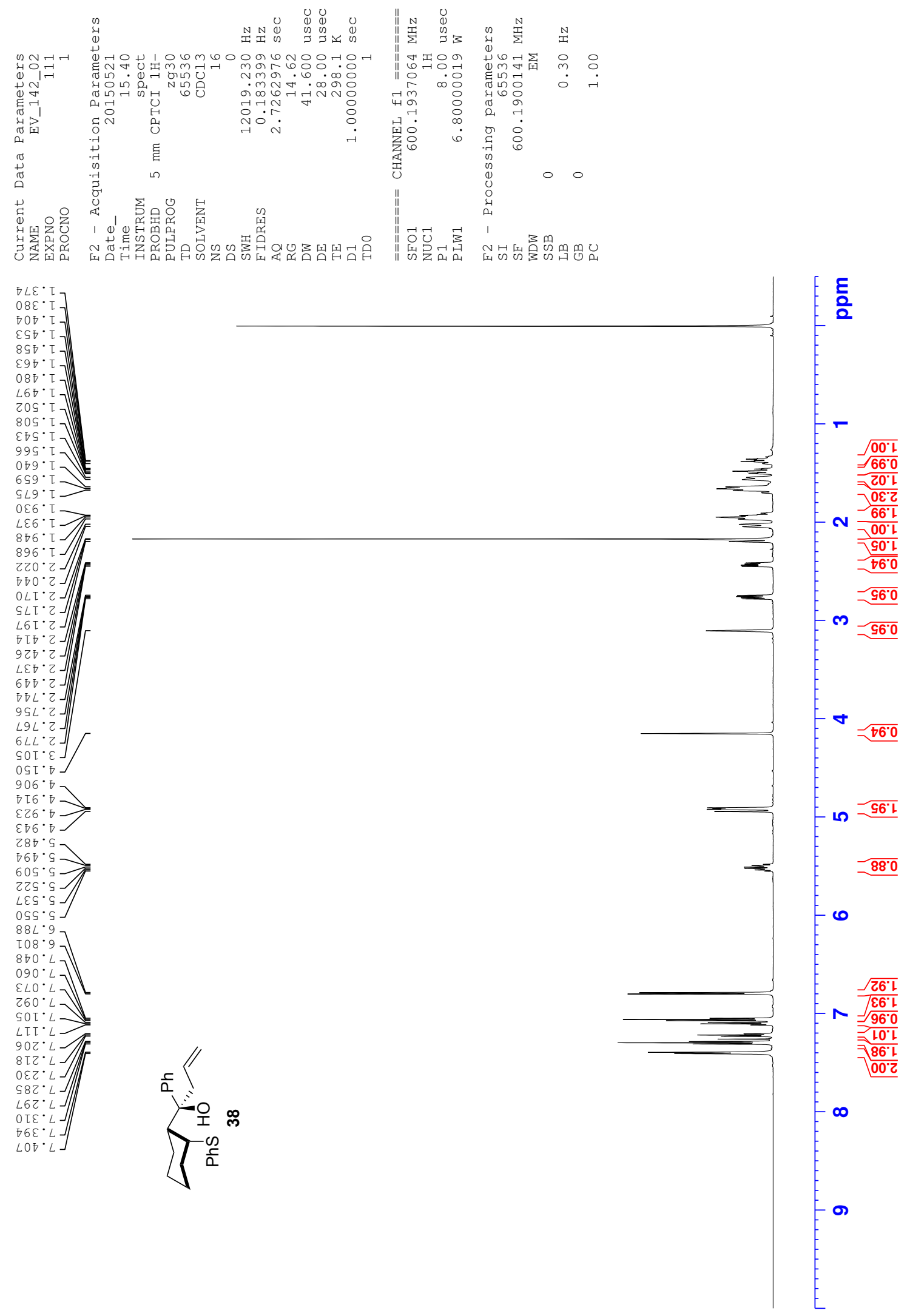

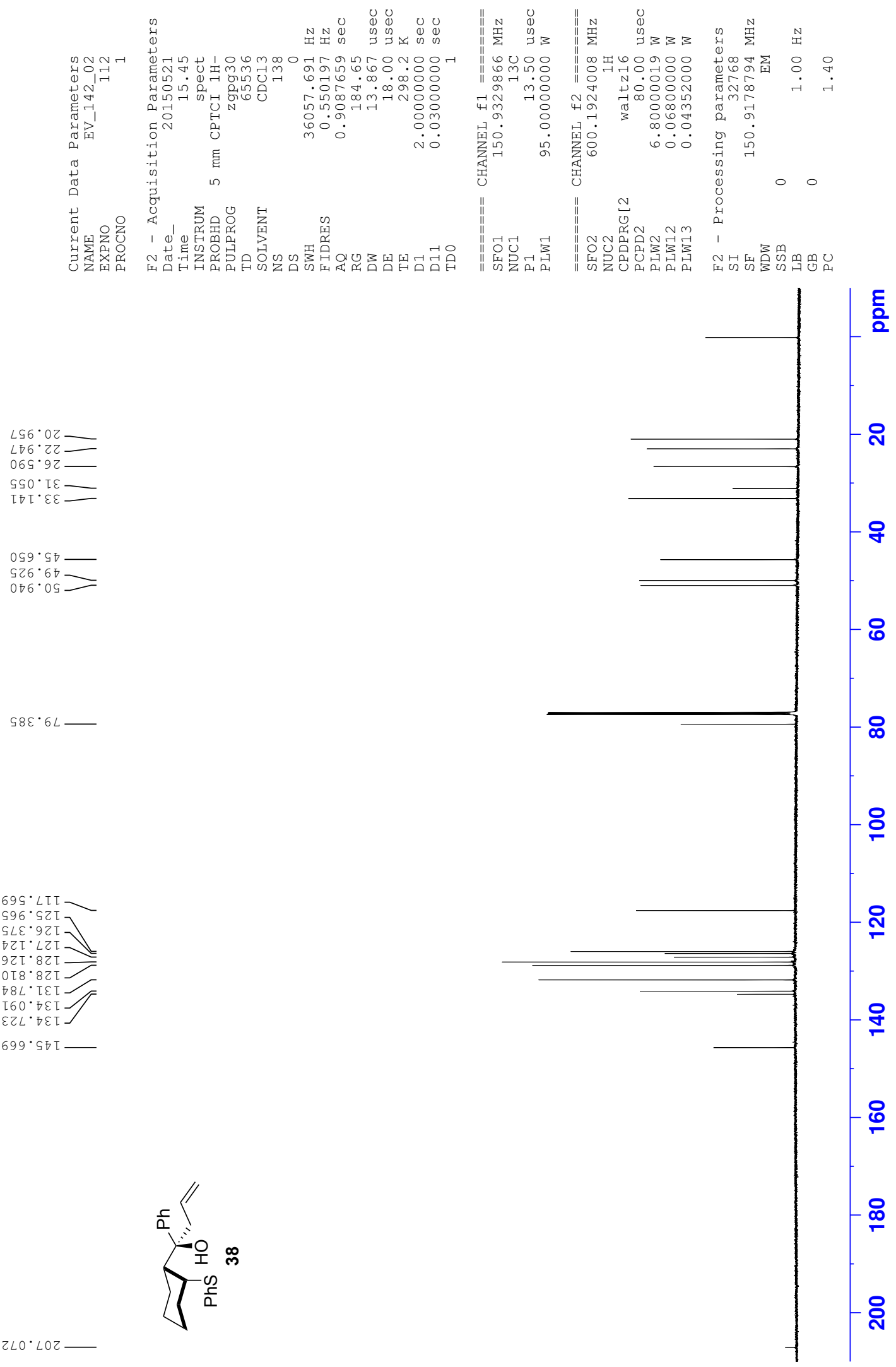

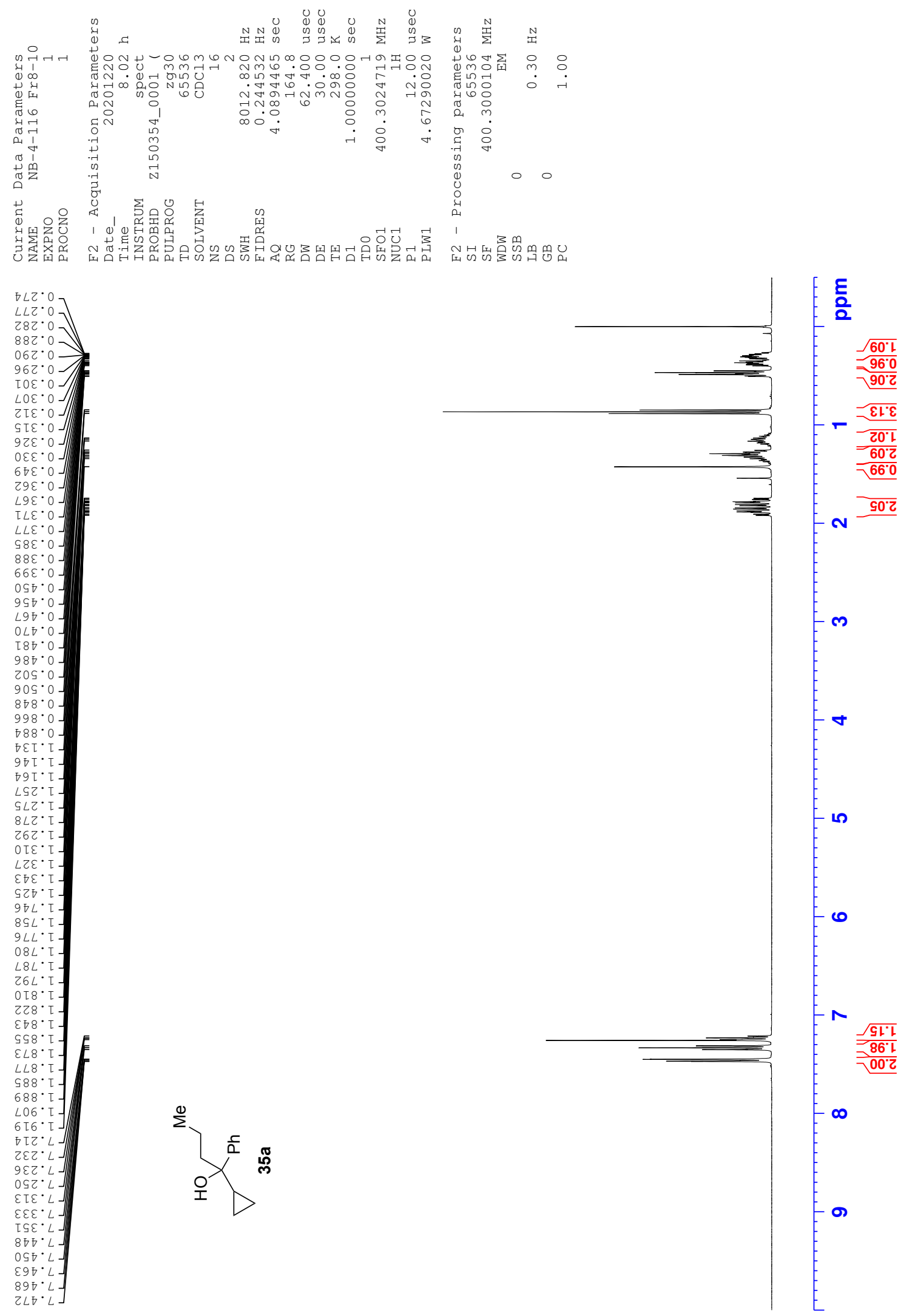

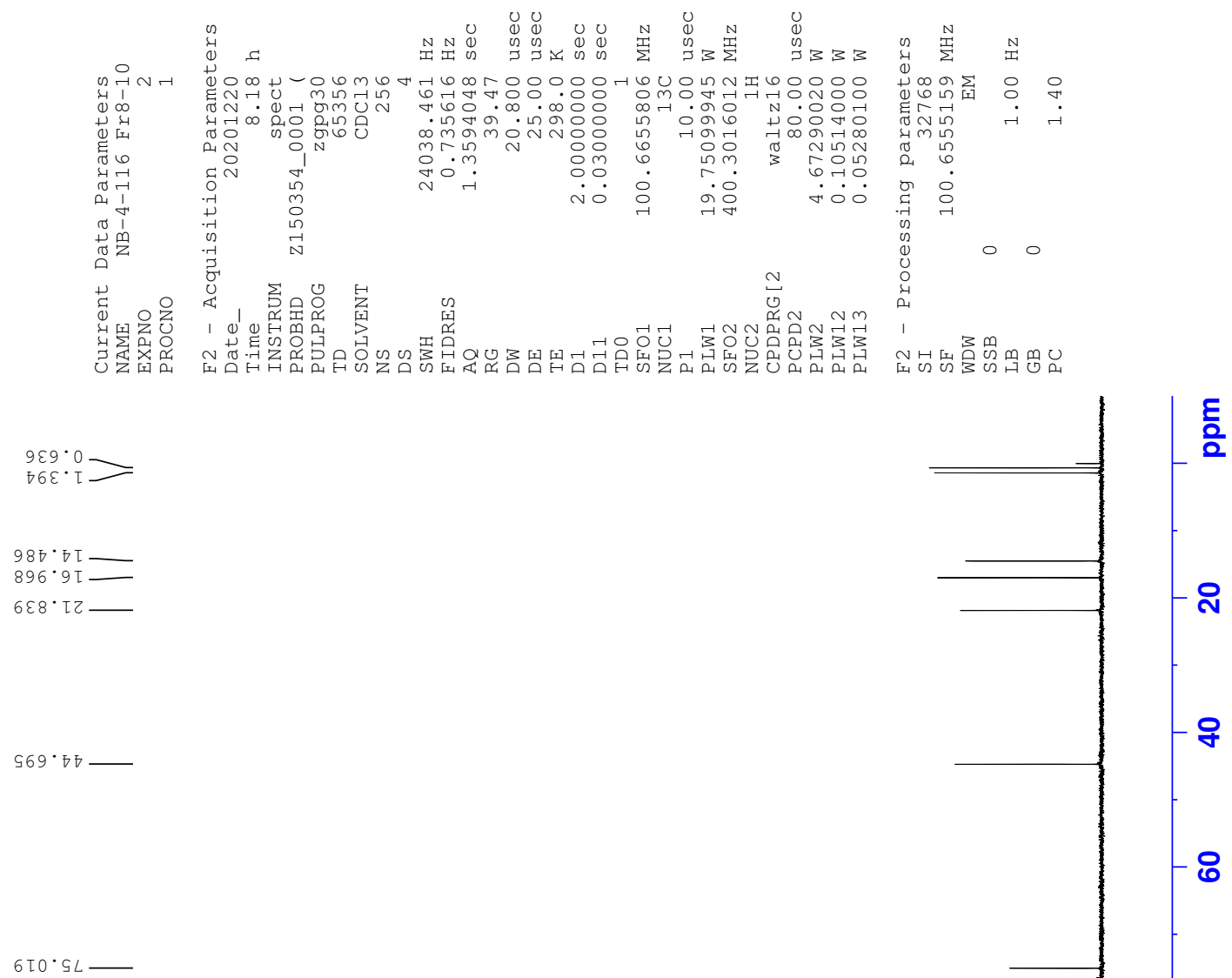

$\infty$

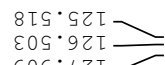

$606^{\circ} \mathrm{LZT} \longrightarrow$

Z8S.97T

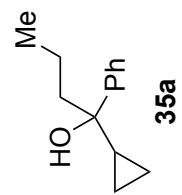

$\stackrel{\circ}{\circ}$

$\stackrel{\infty}{\infty}$

ঃั 

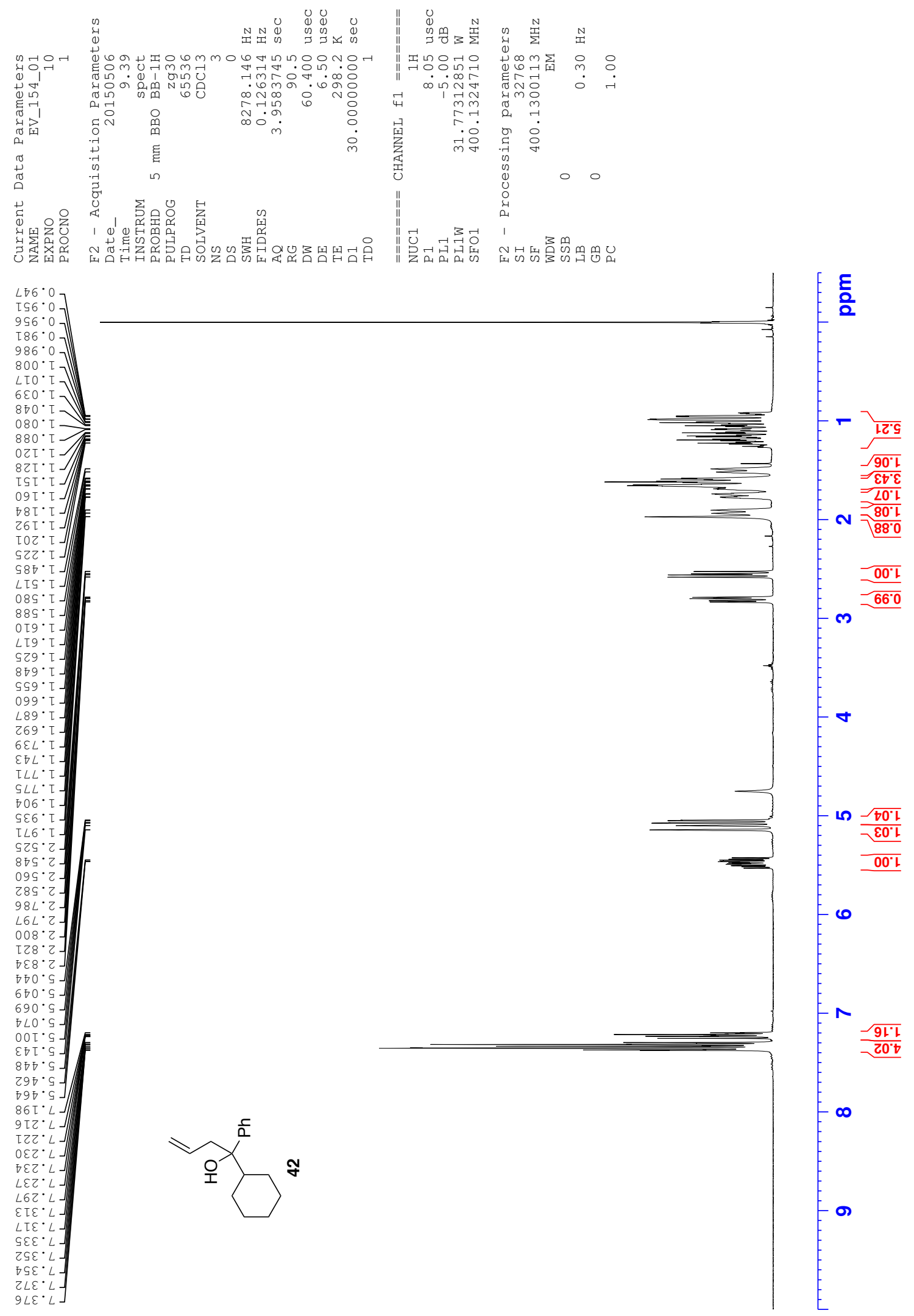

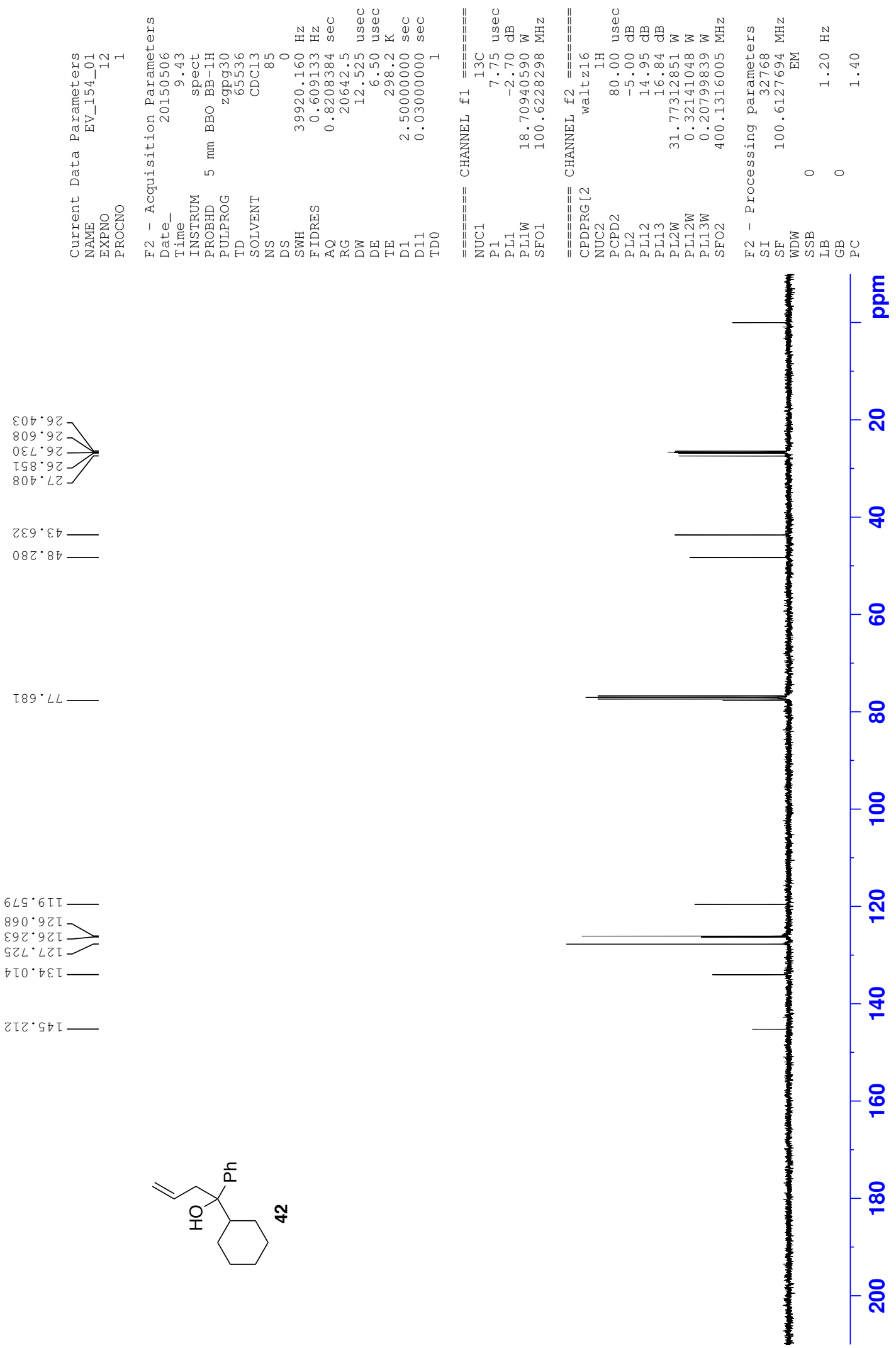

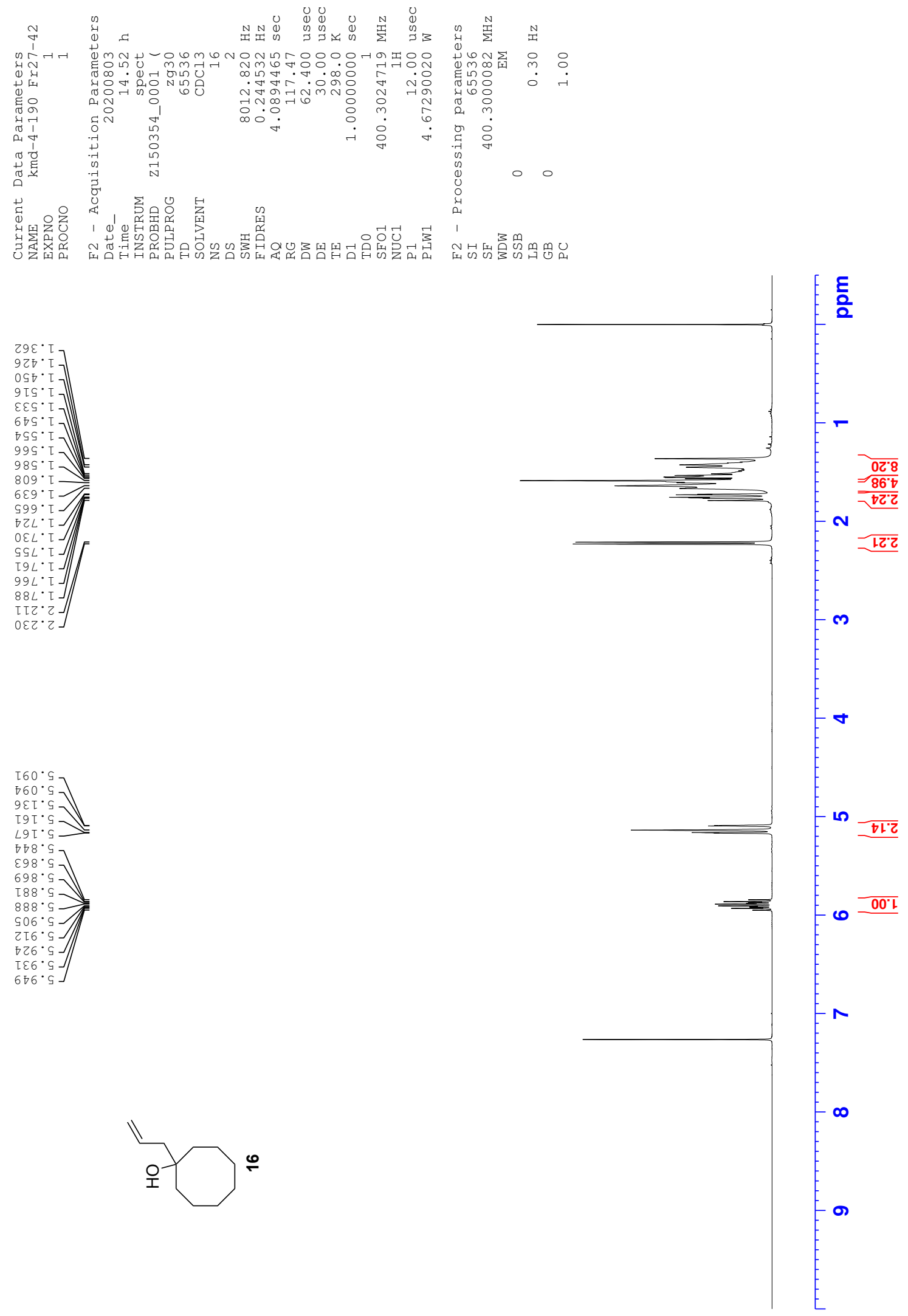

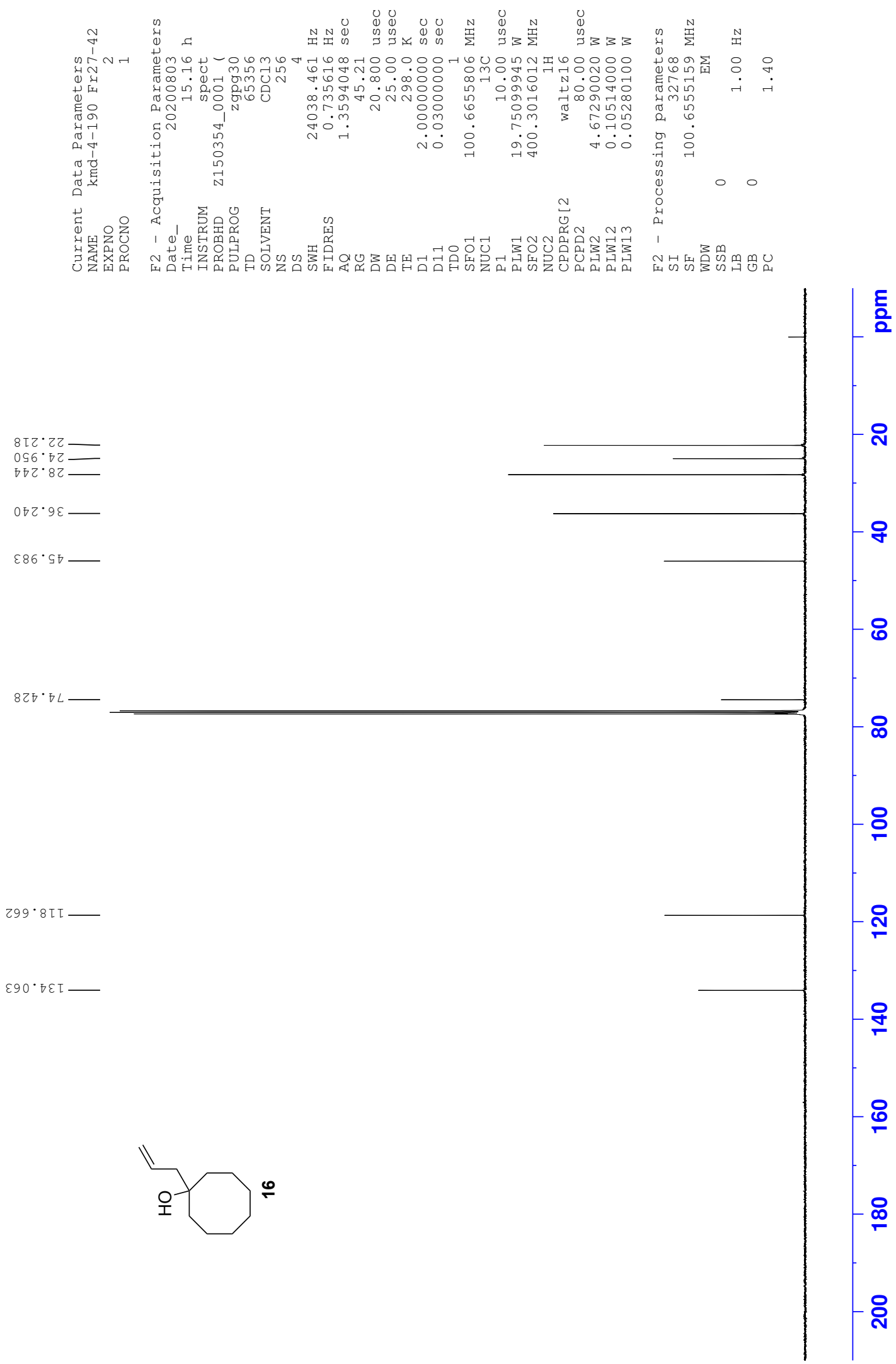

틍 

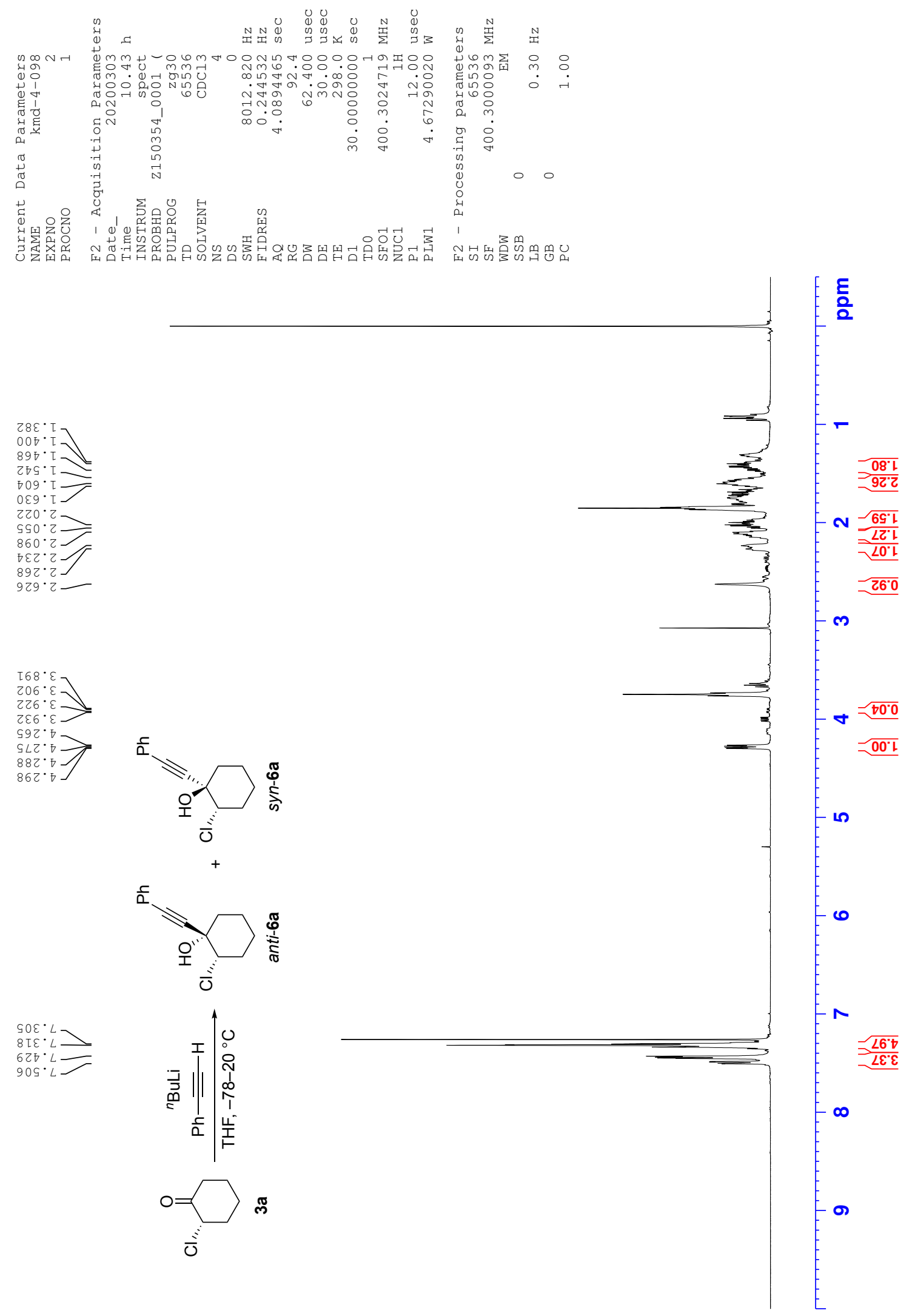

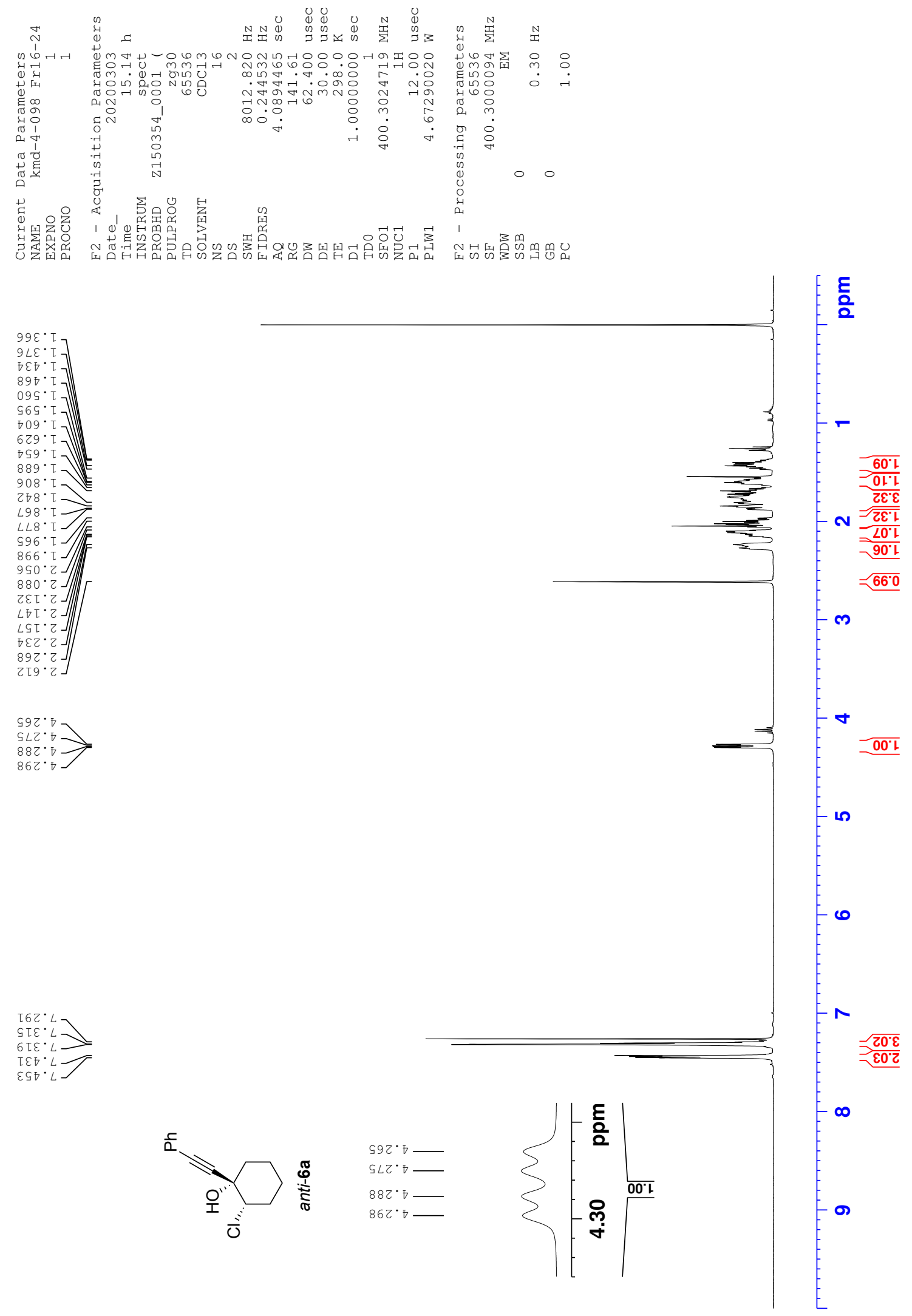

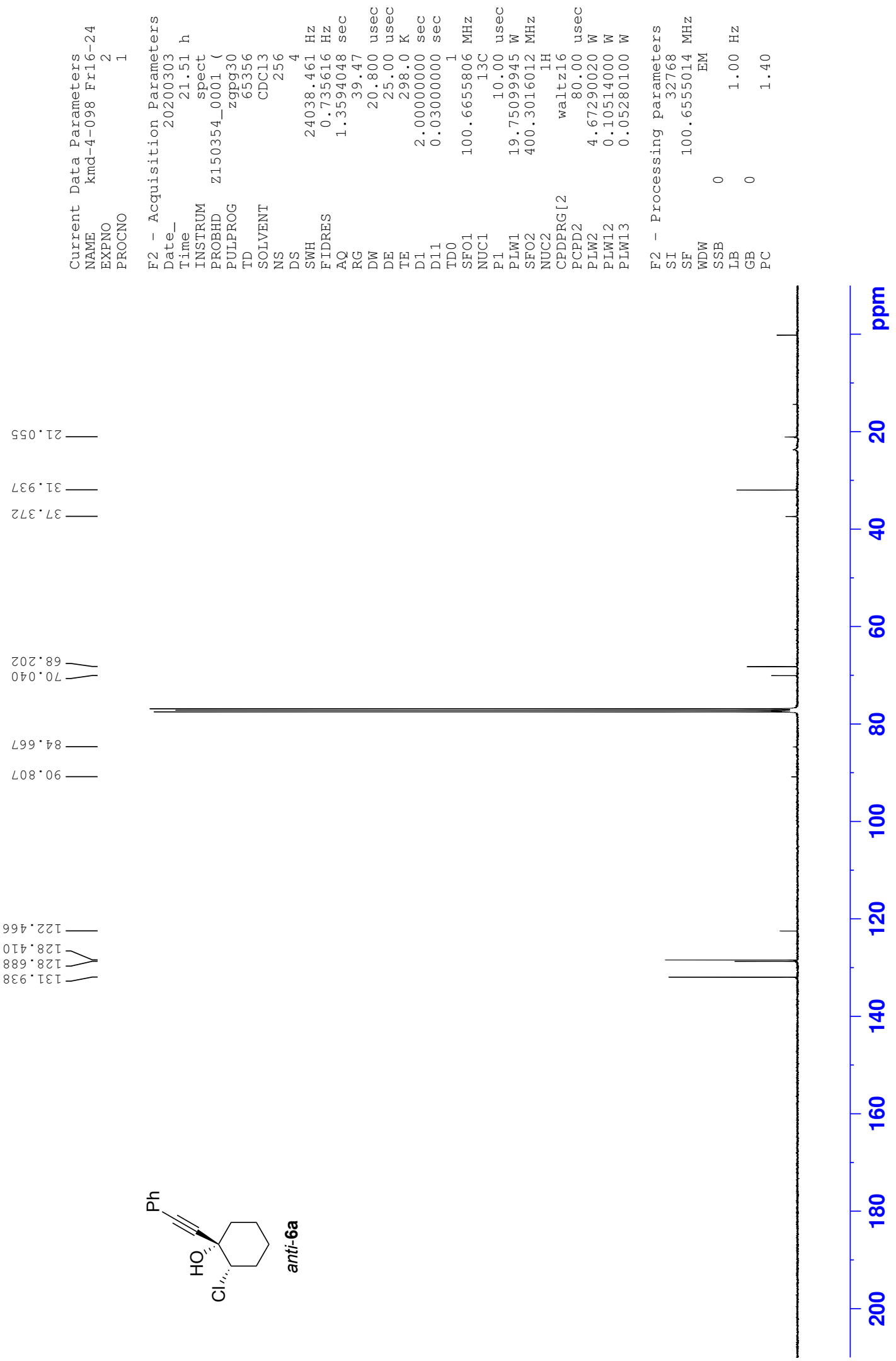

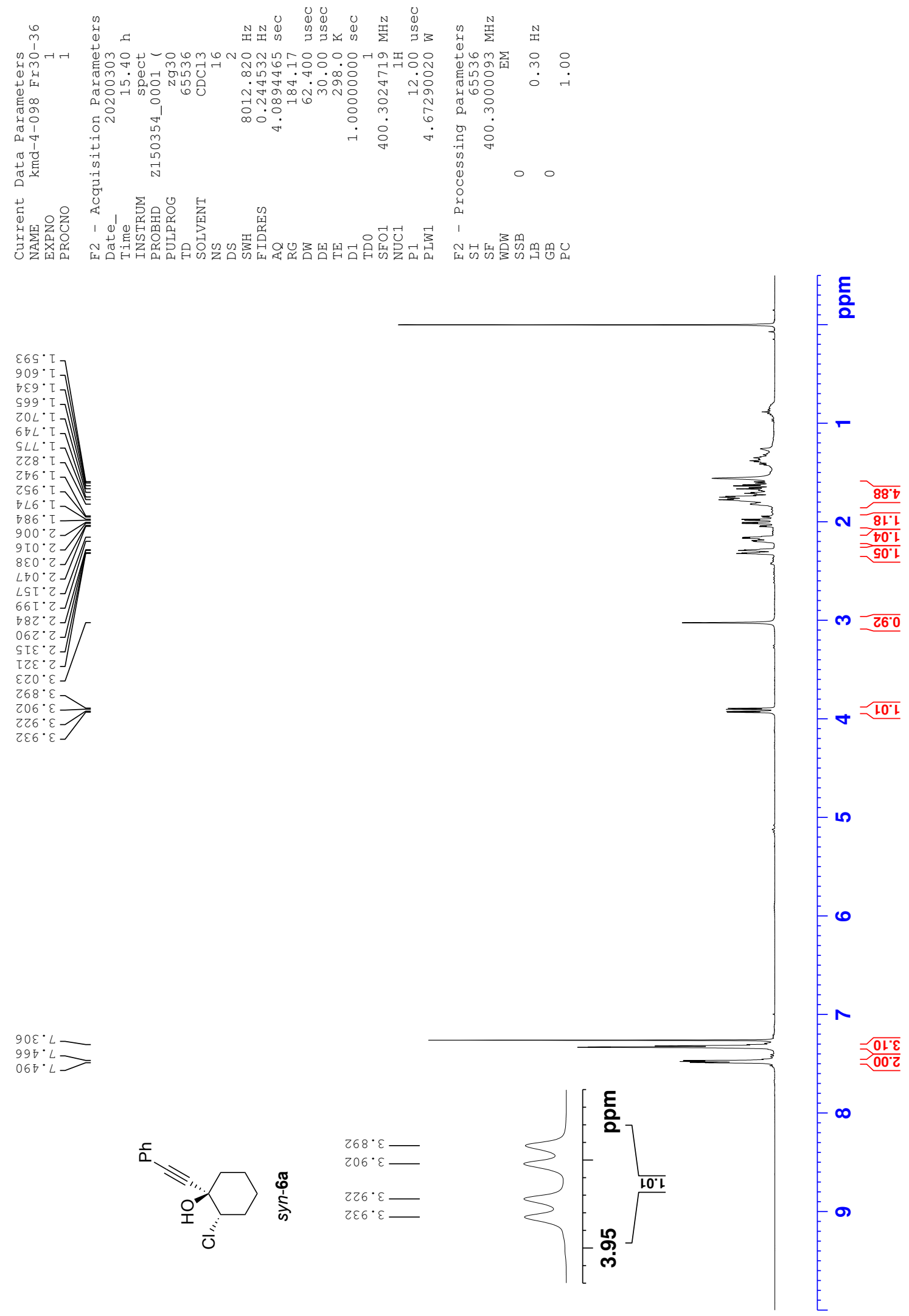

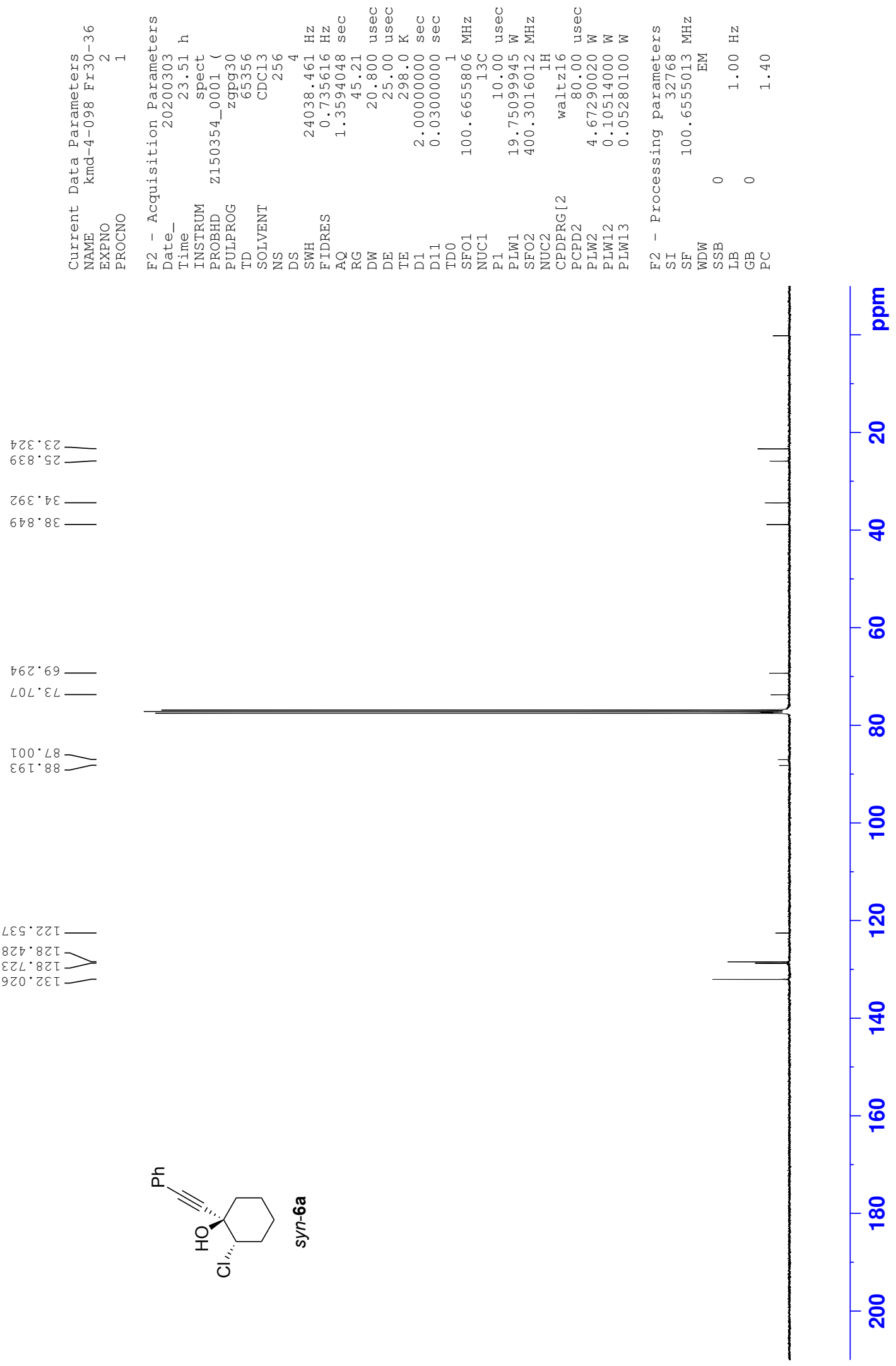

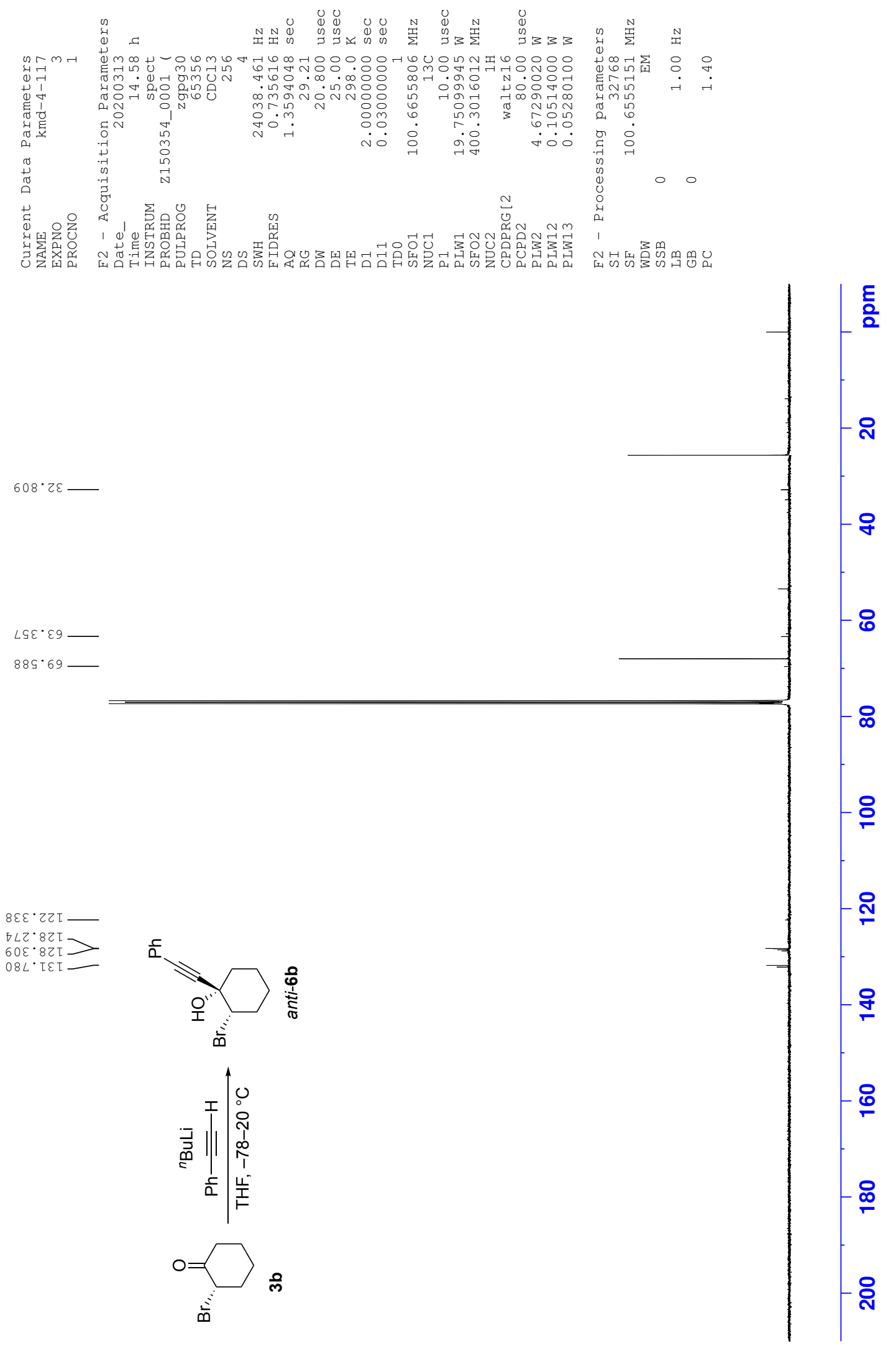

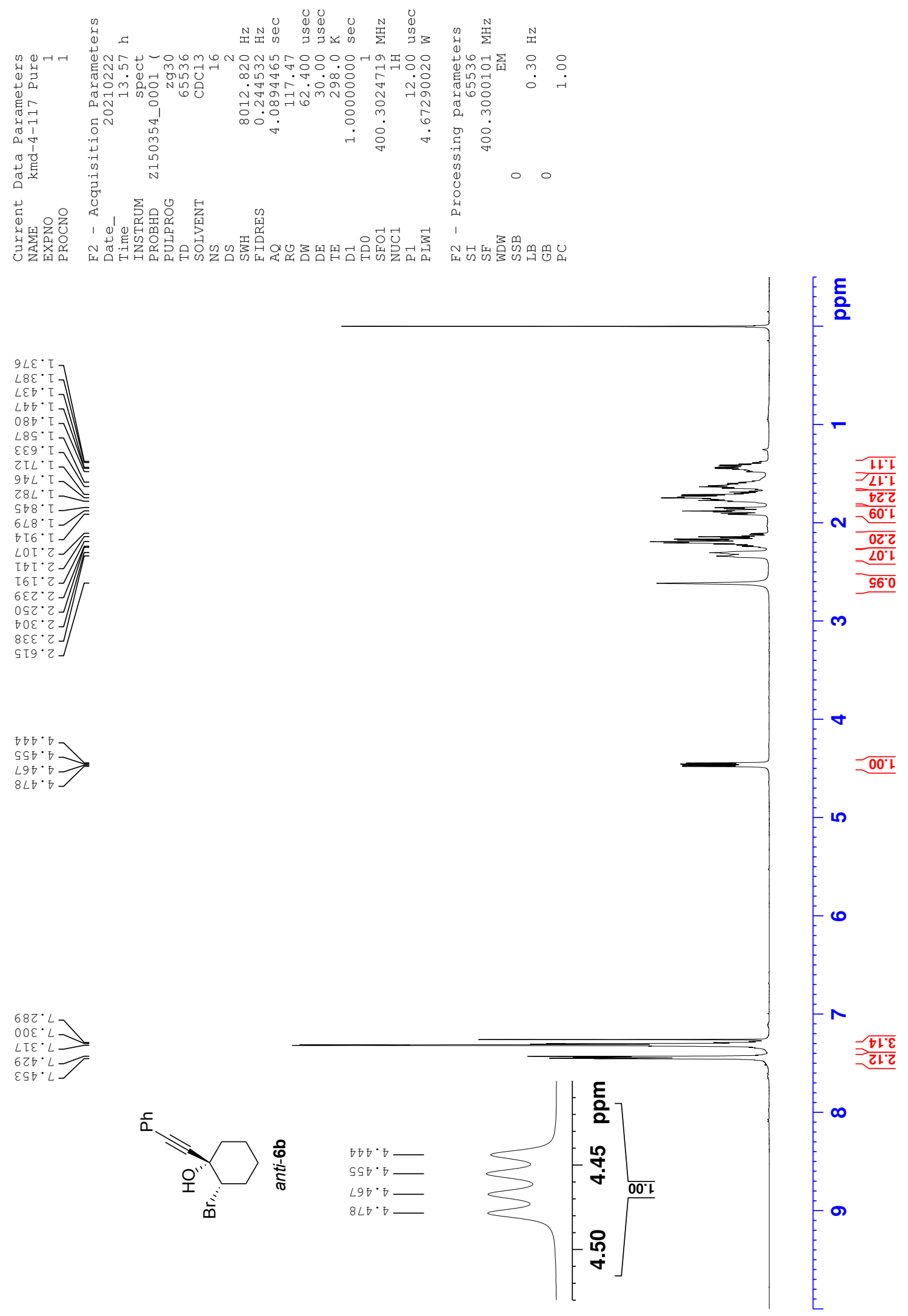

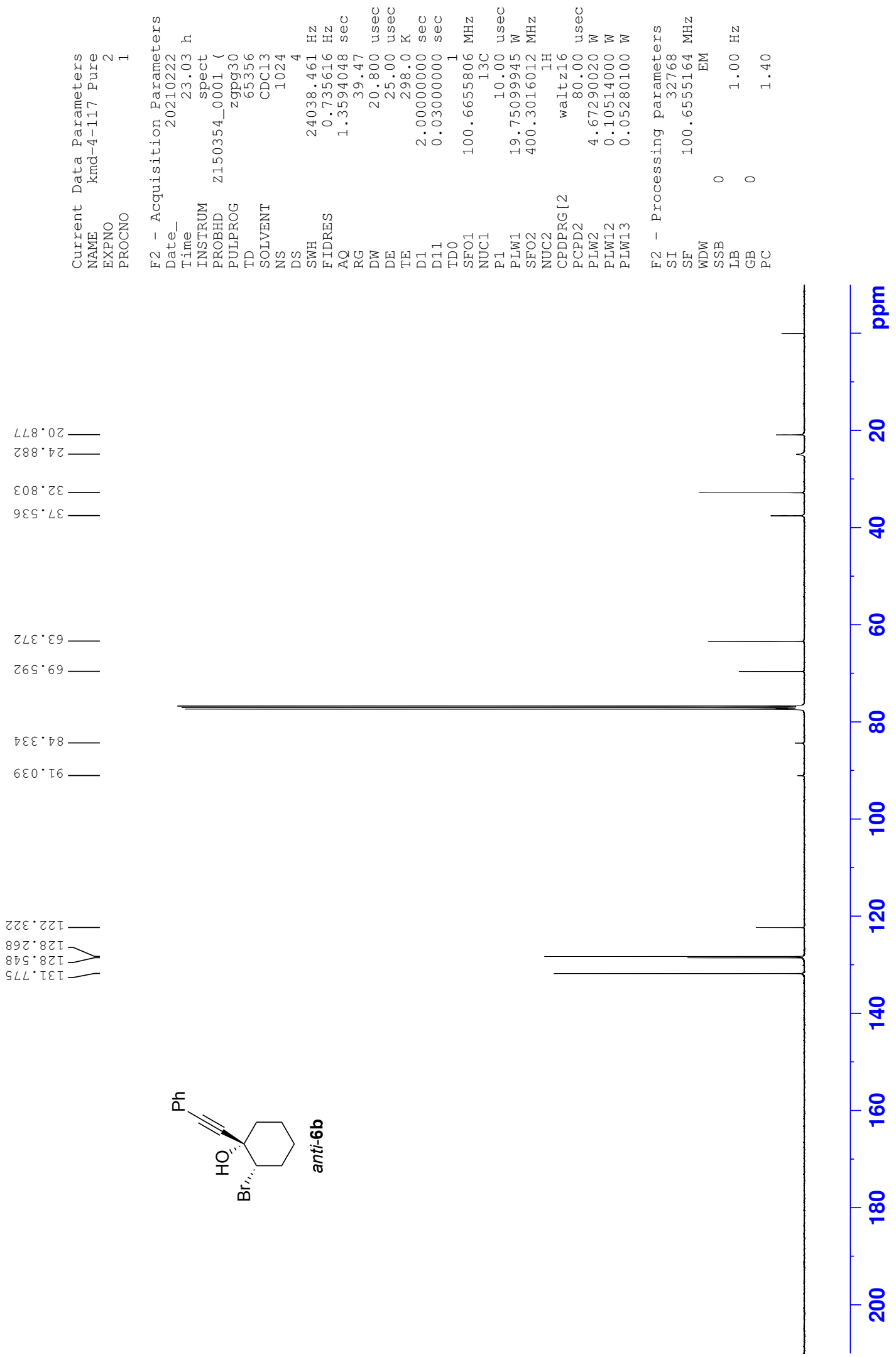

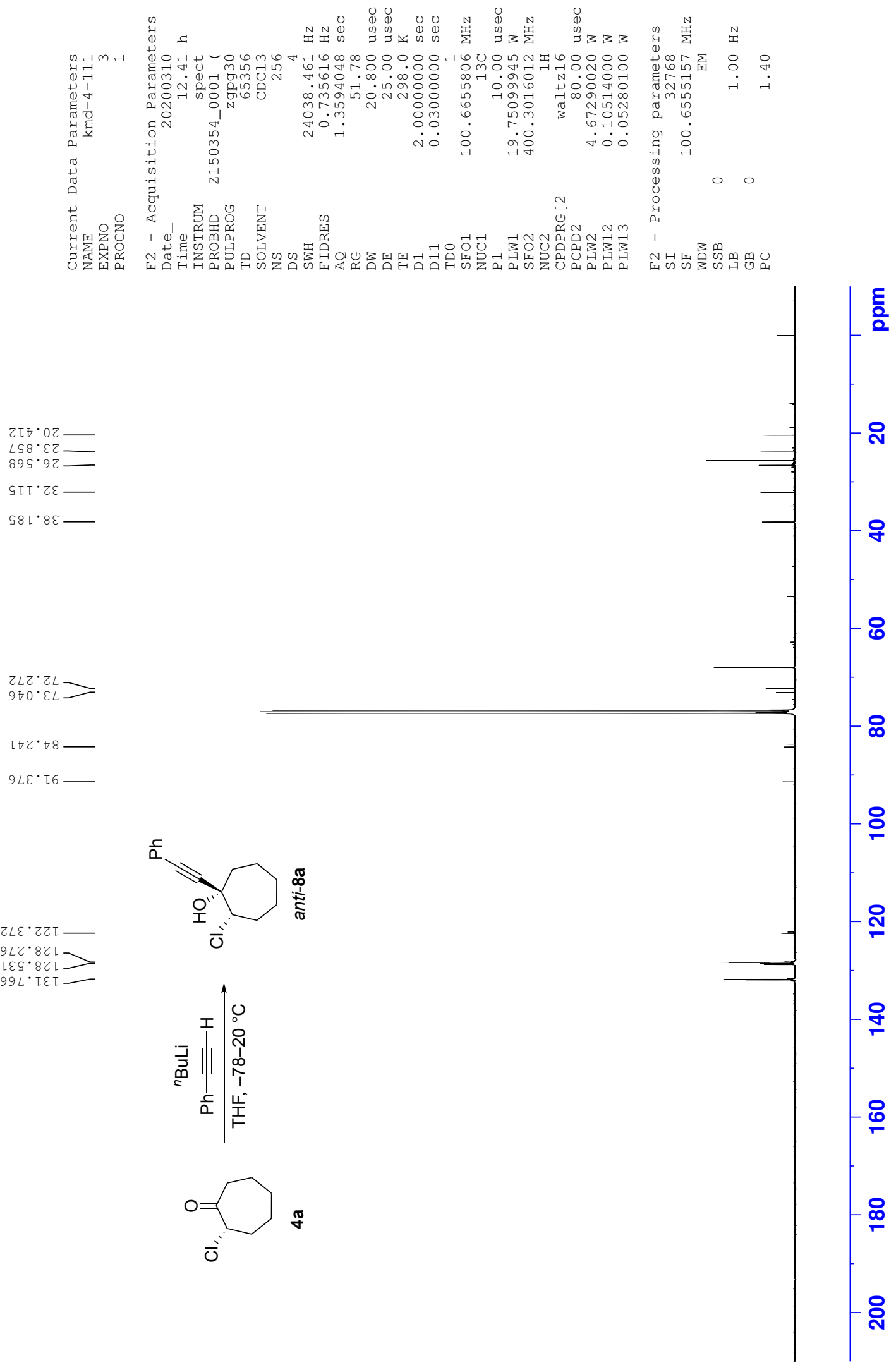

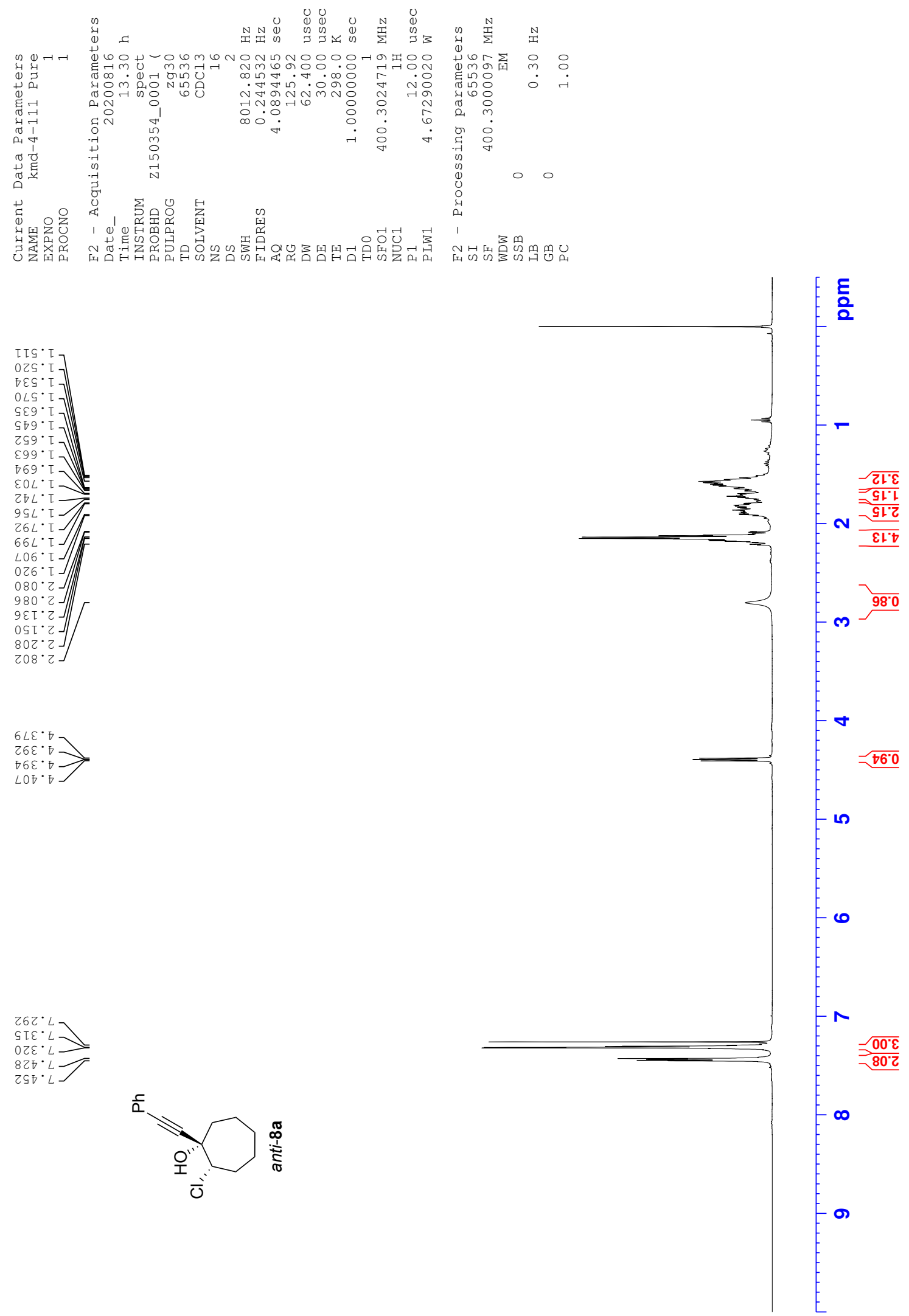

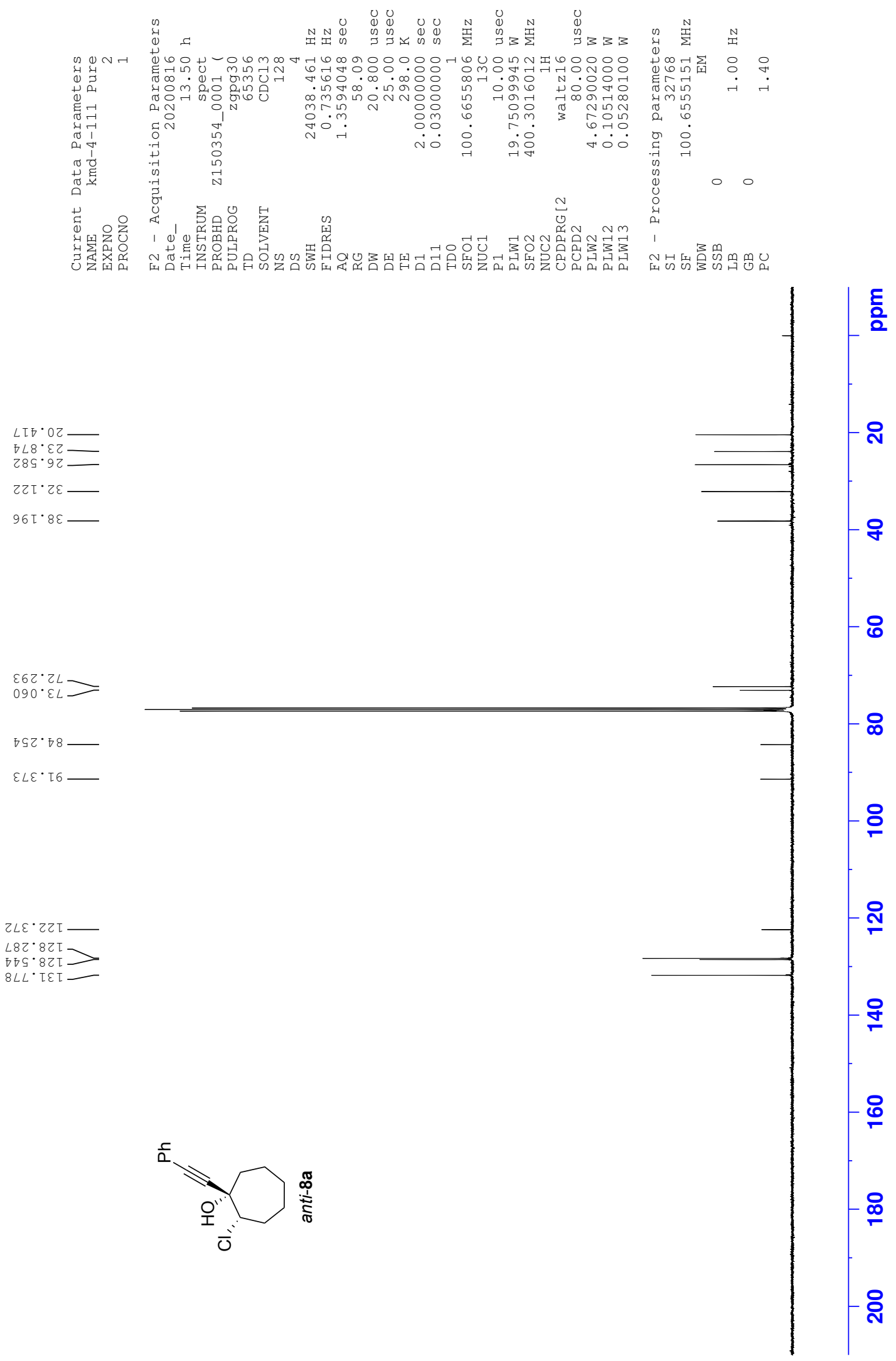

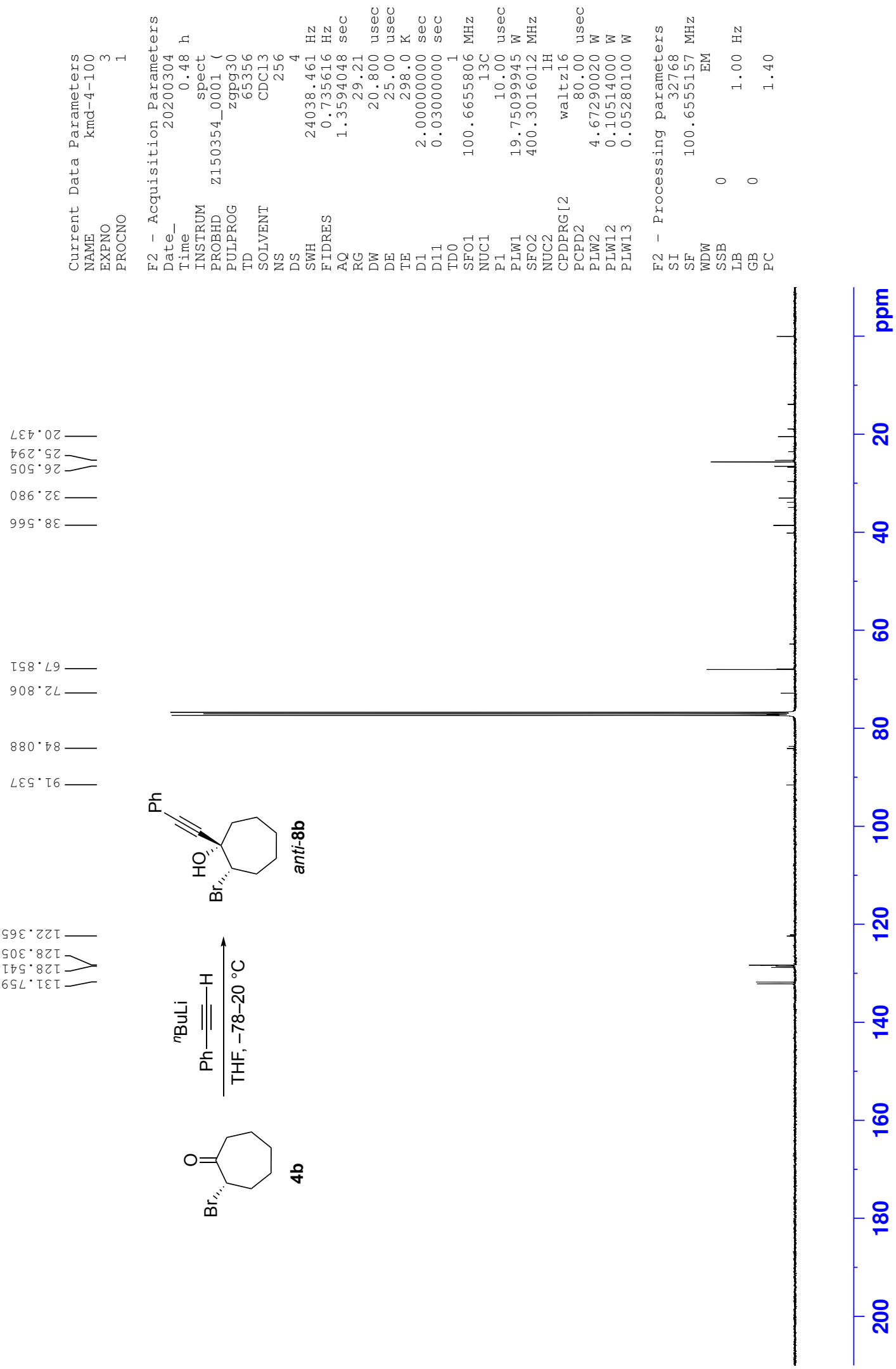

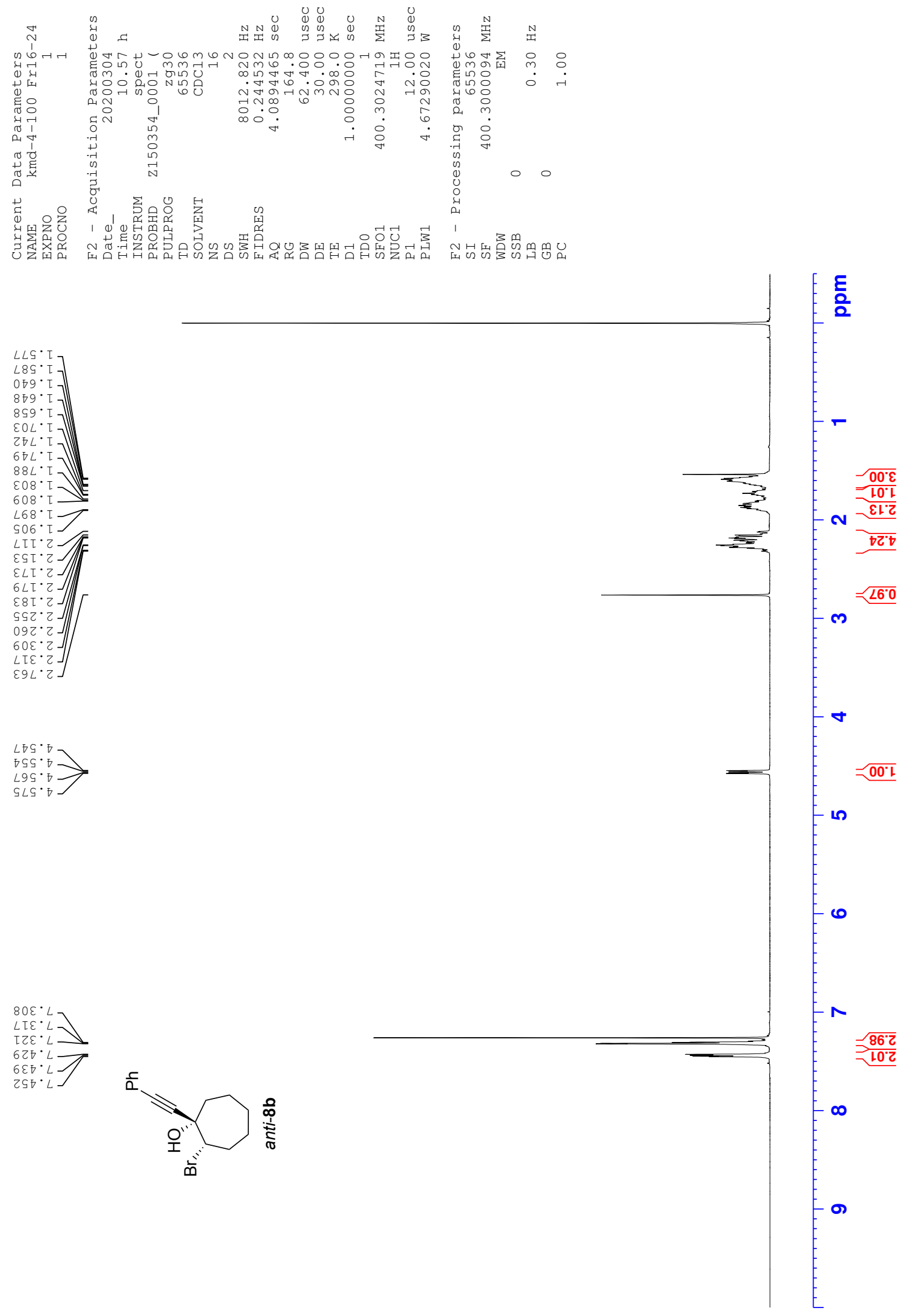

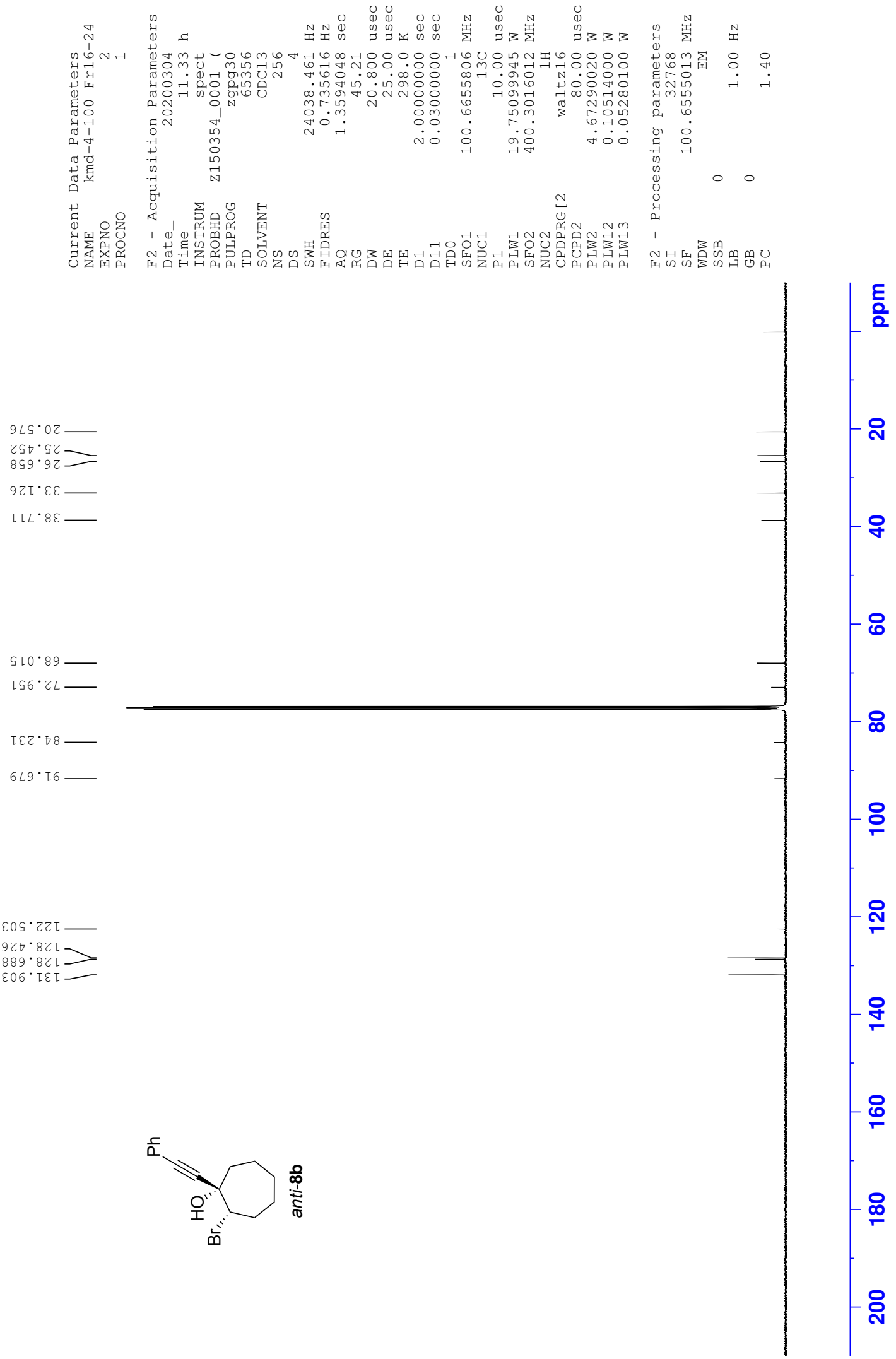

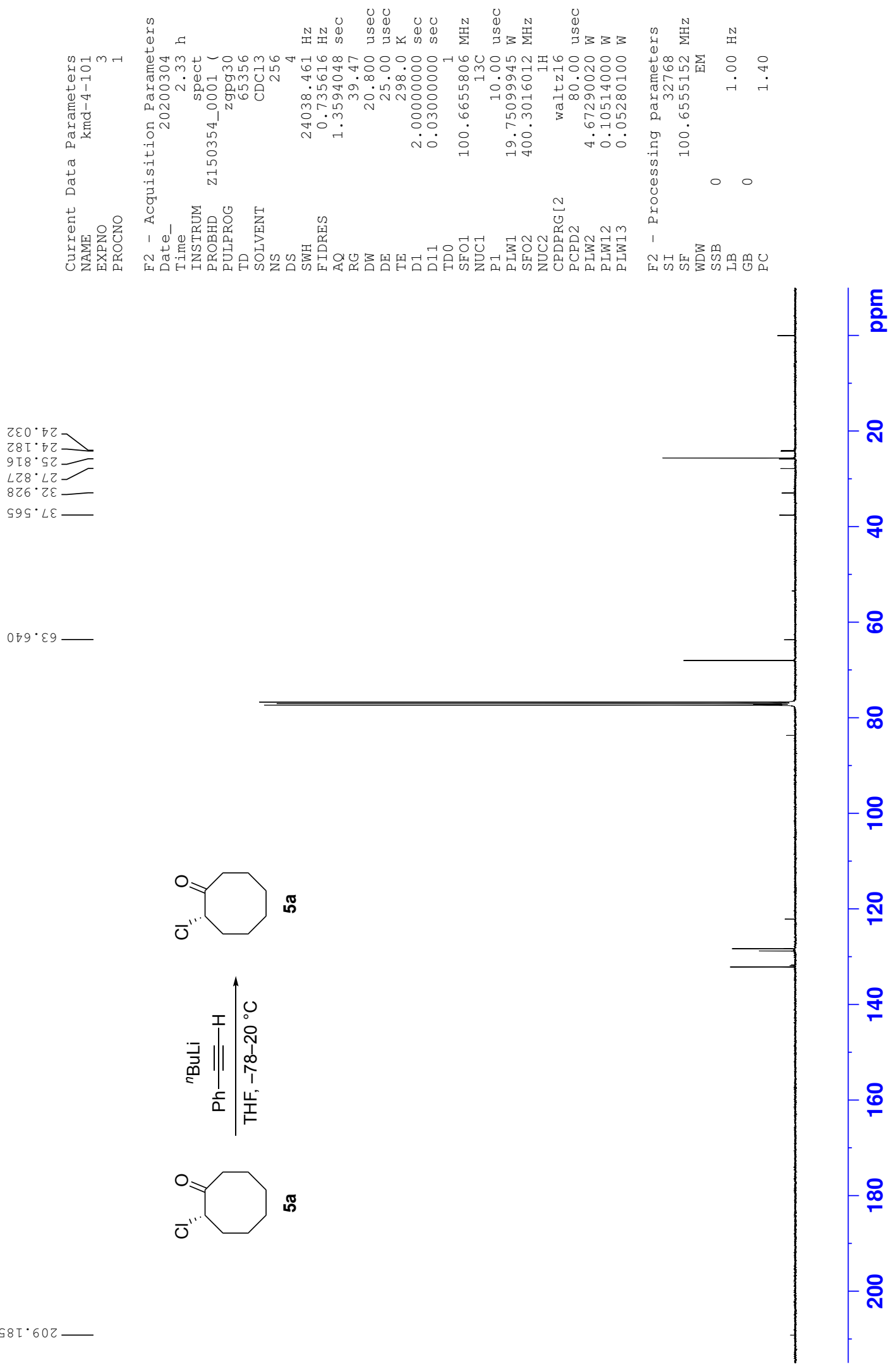

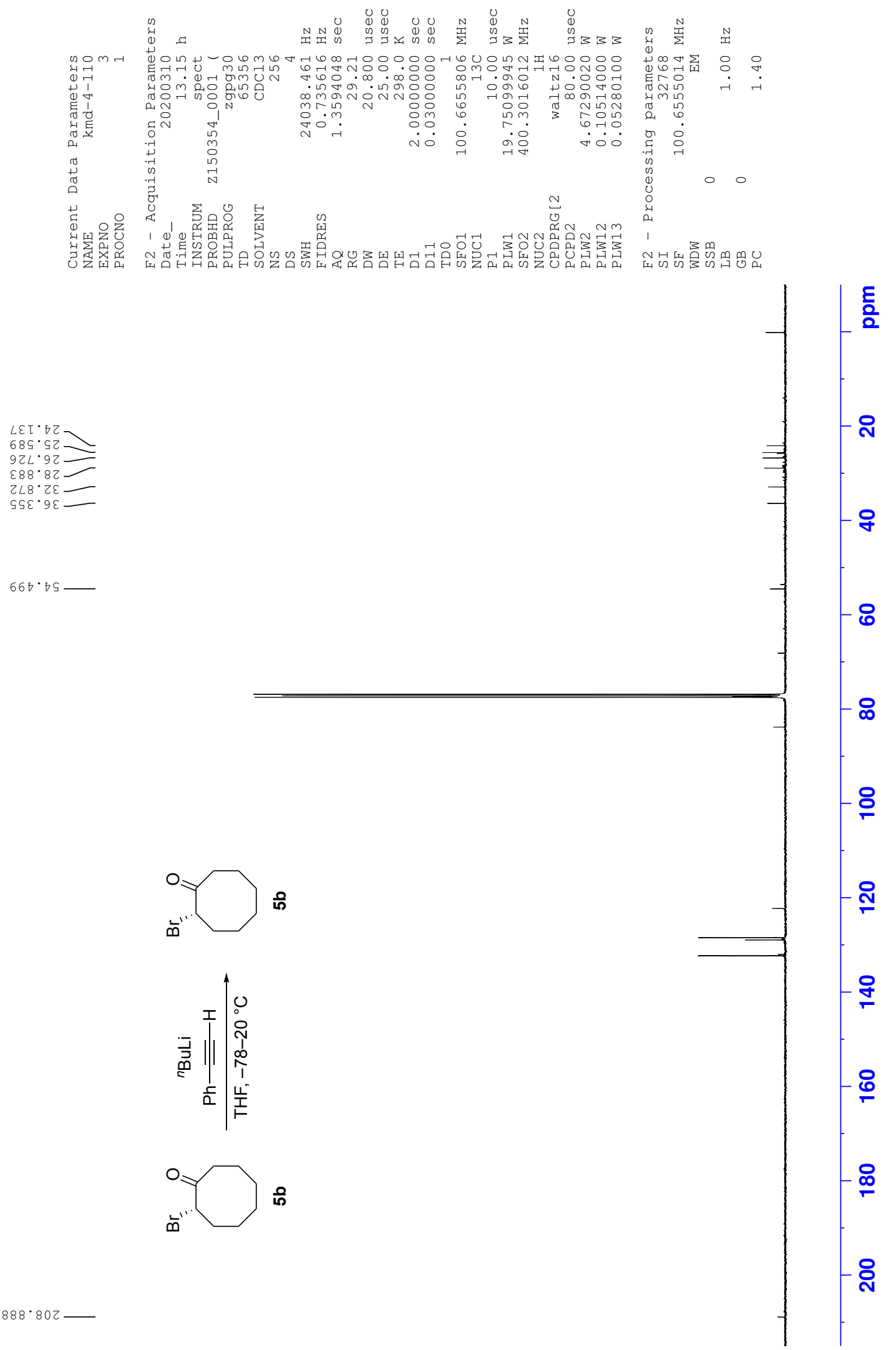

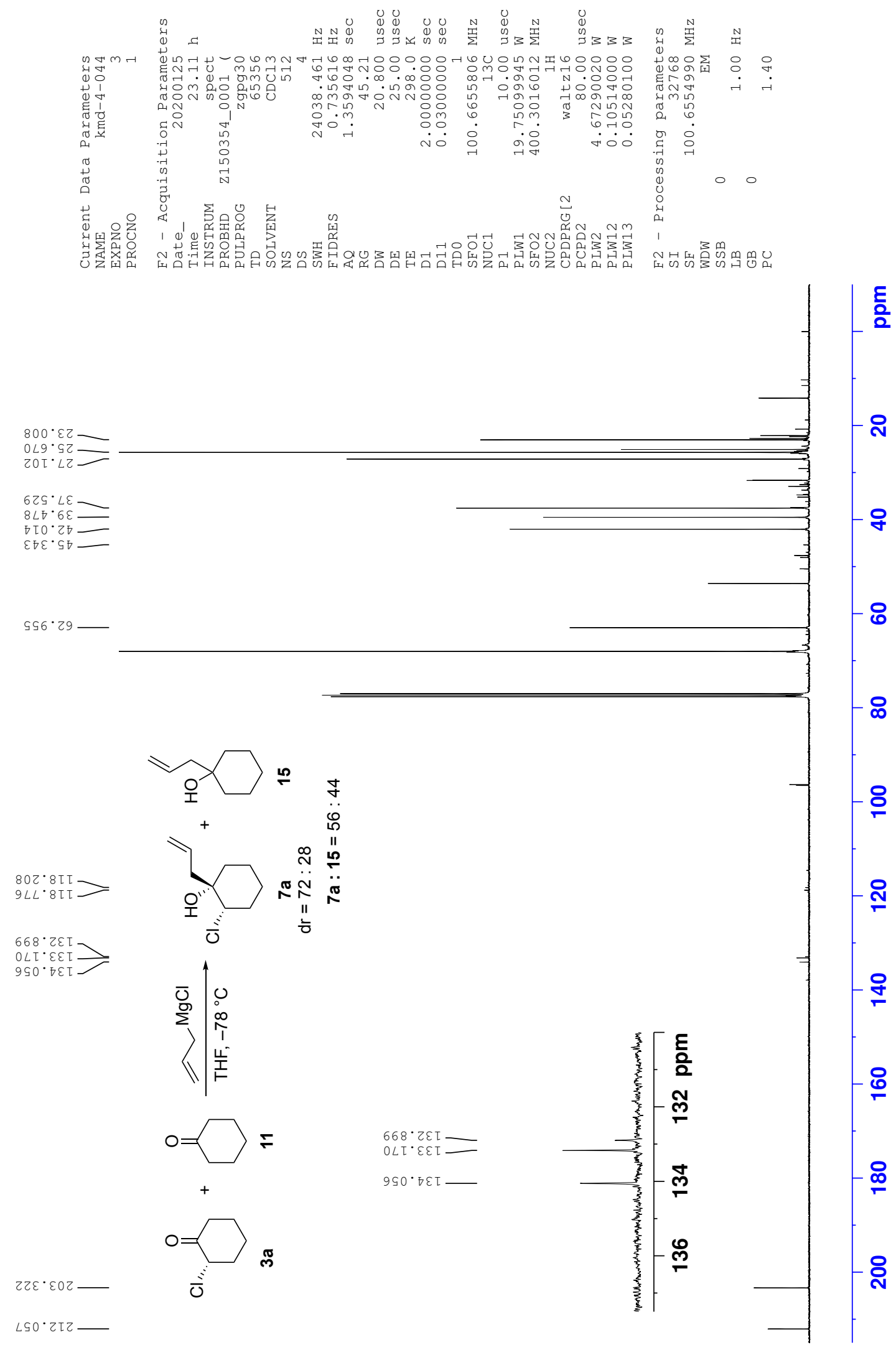

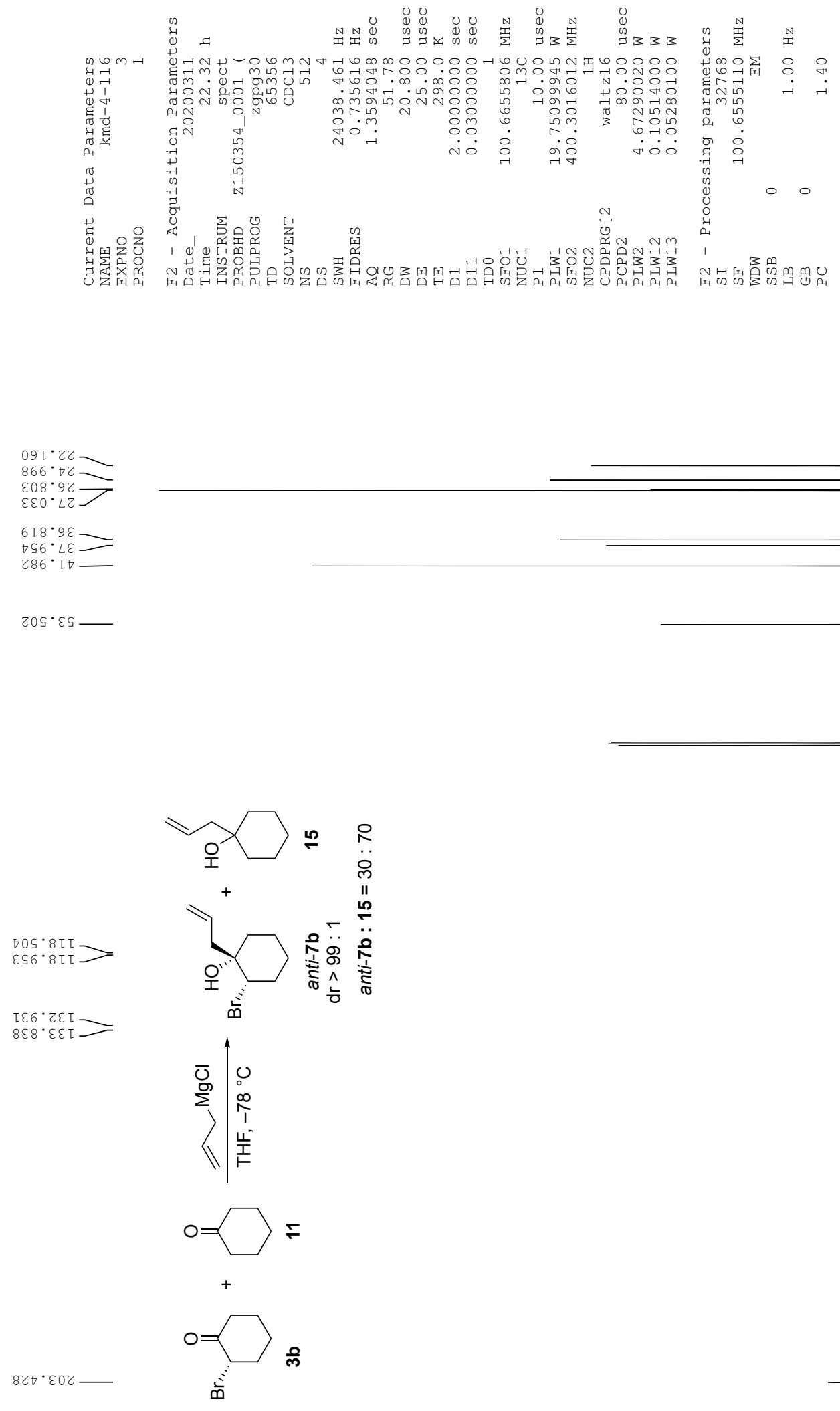

틀

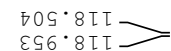

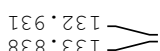
$8 \varepsilon 8^{\circ} \varepsilon \varepsilon \tau$

$8 z \hbar^{\circ} \varepsilon 0 z$ ตั

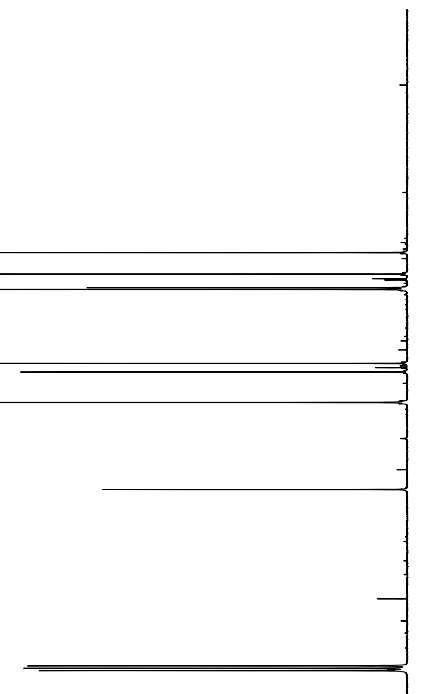

- ำ

- 우

8

$\infty$

음

- 오

움

$\stackrel{8}{\underline{C}}$

$\stackrel{\infty}{\oplus}$

오 

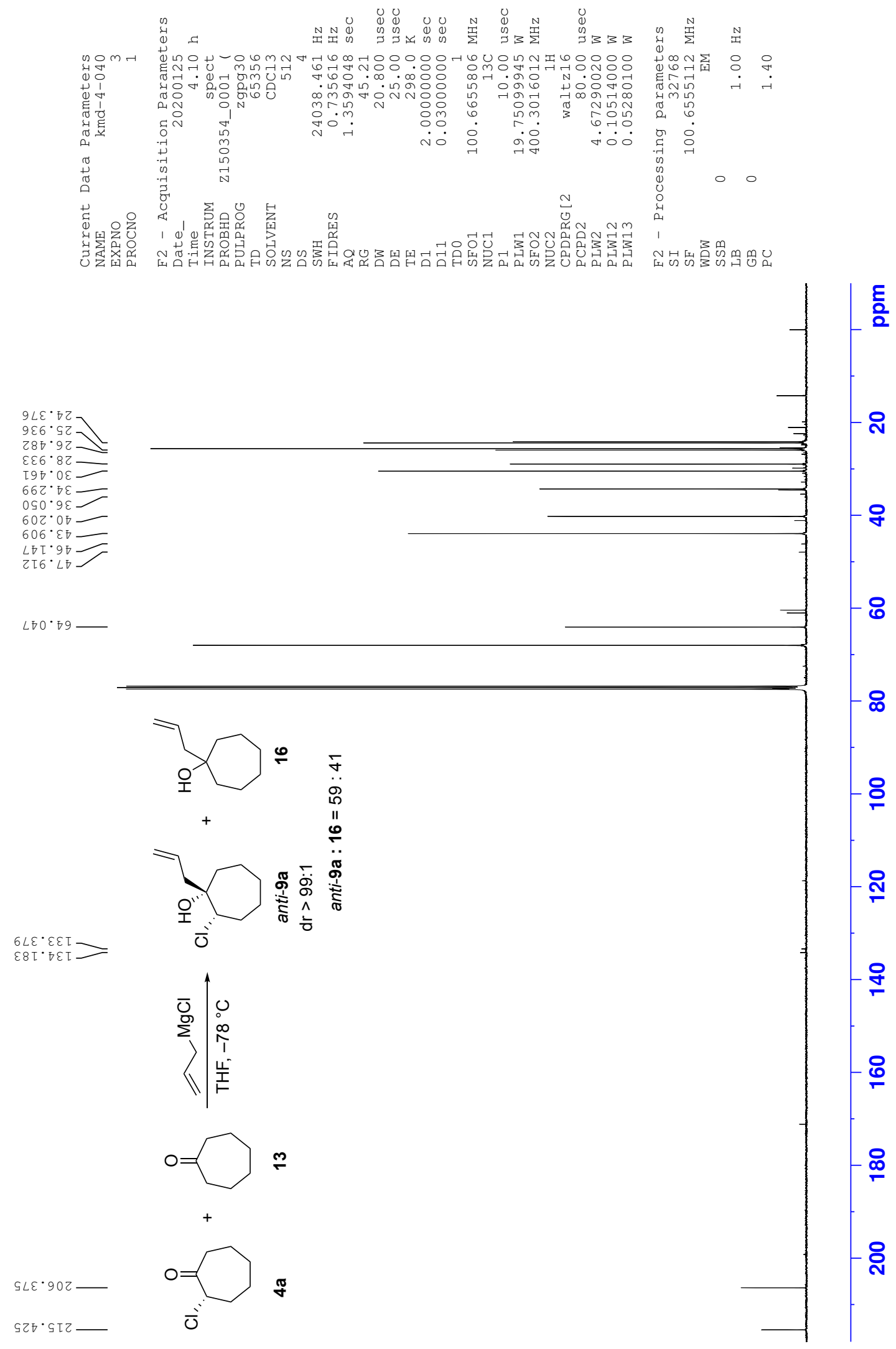

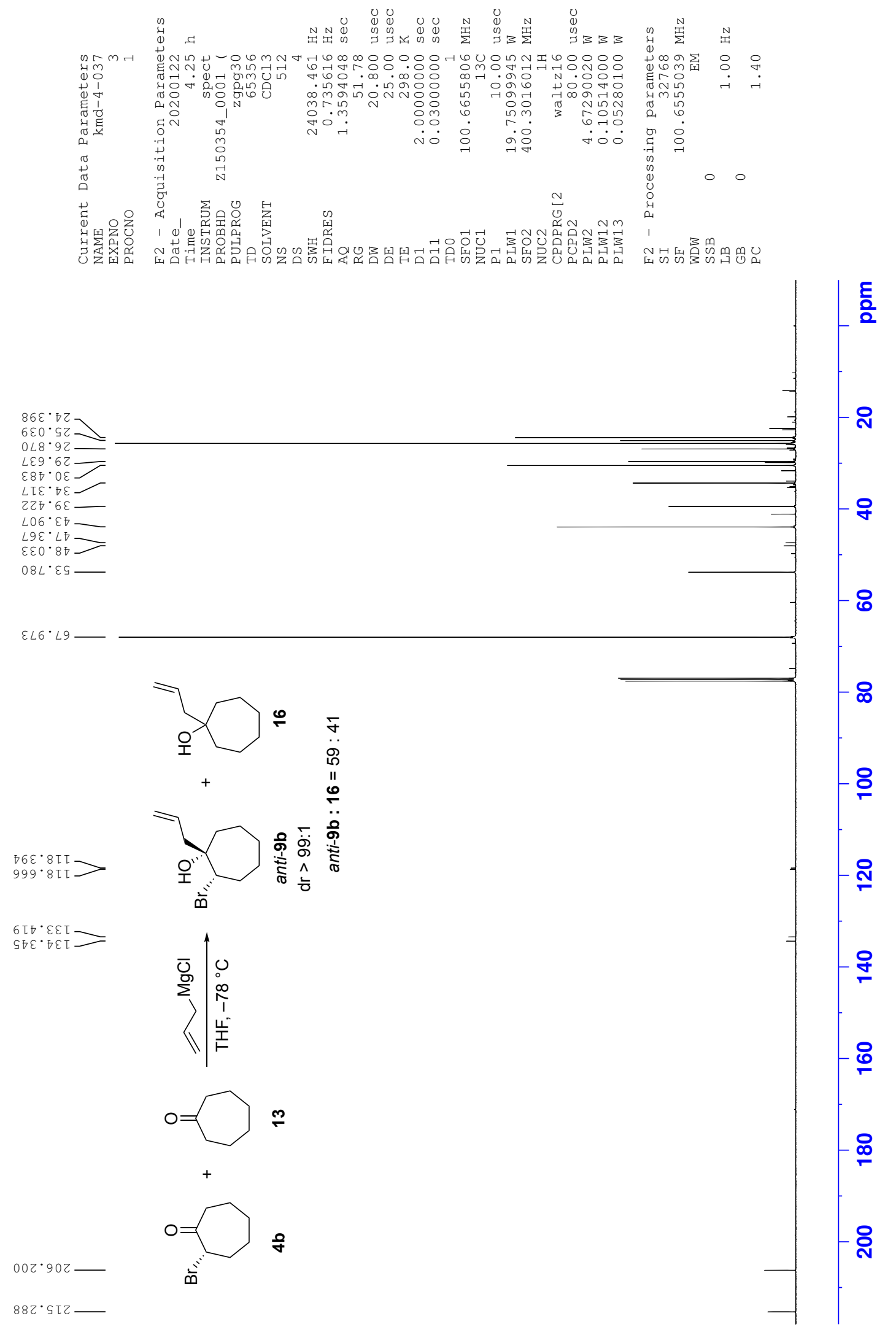

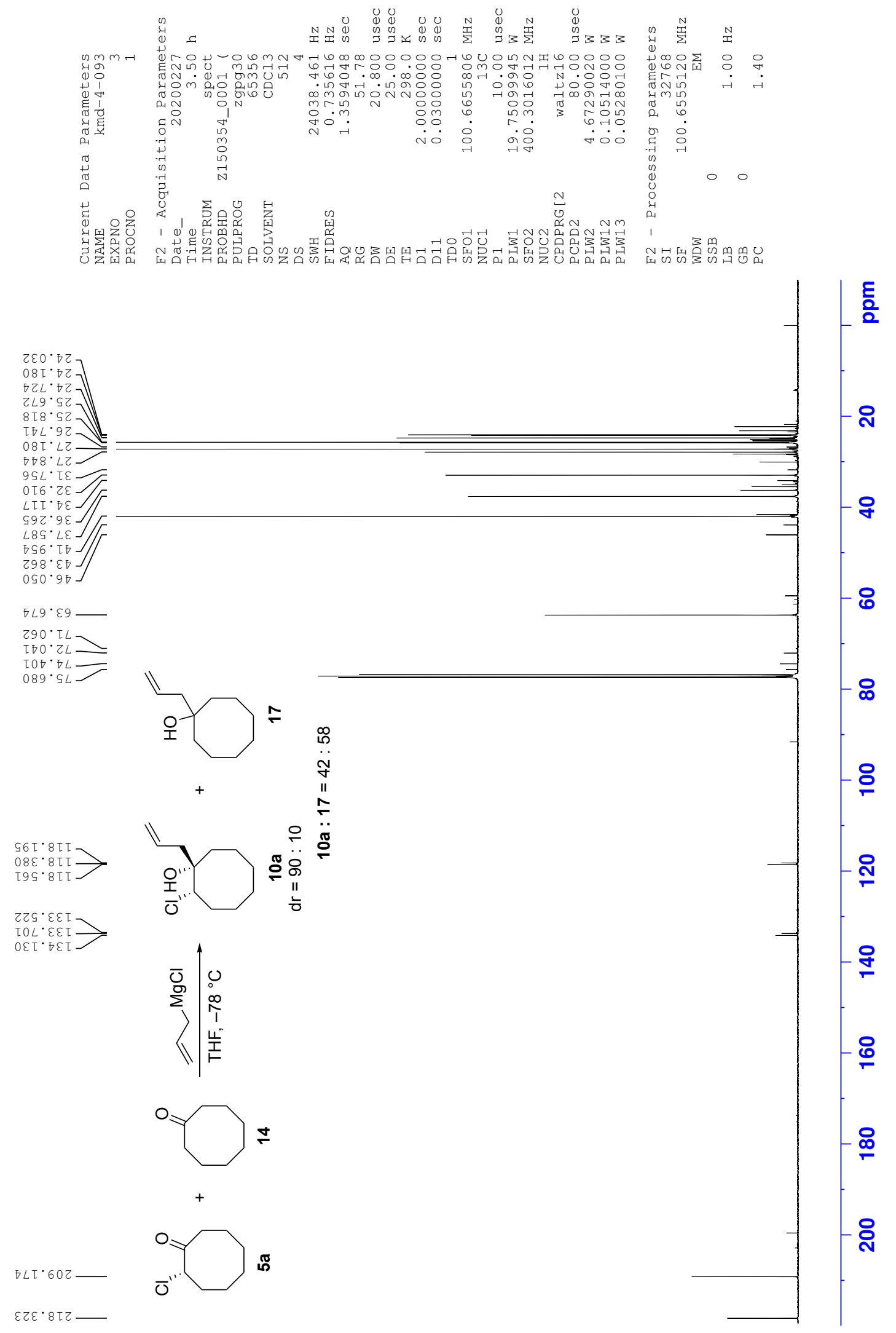

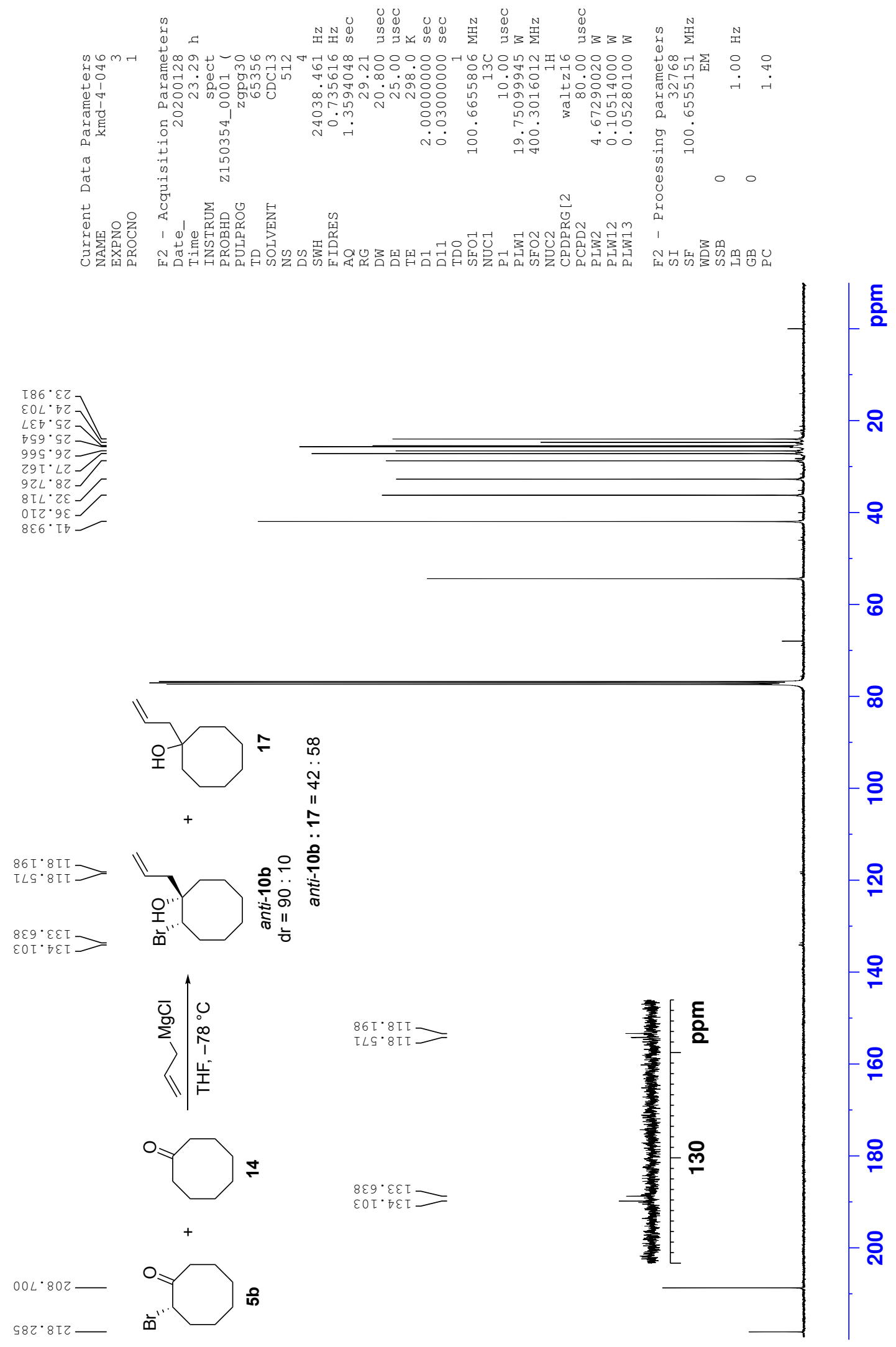

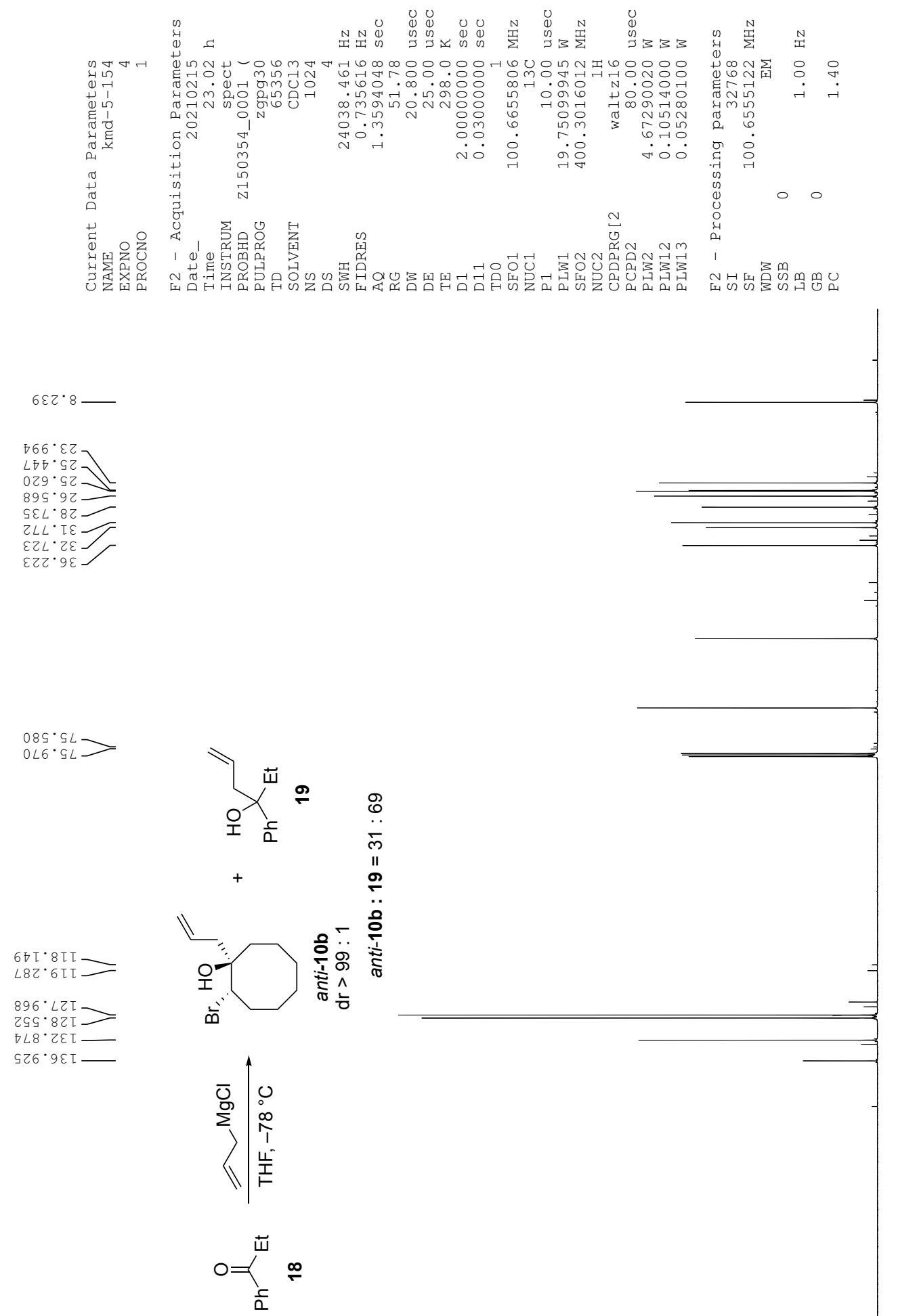

틍
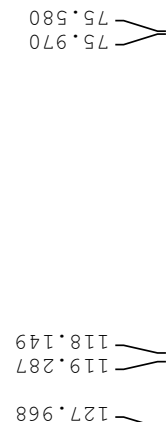

$295 \cdot 8 Z T$

$\operatorname{LS} \cdot 2 \varepsilon[$

$\varsigma 26^{\circ} 9 \varepsilon \mathrm{L}$
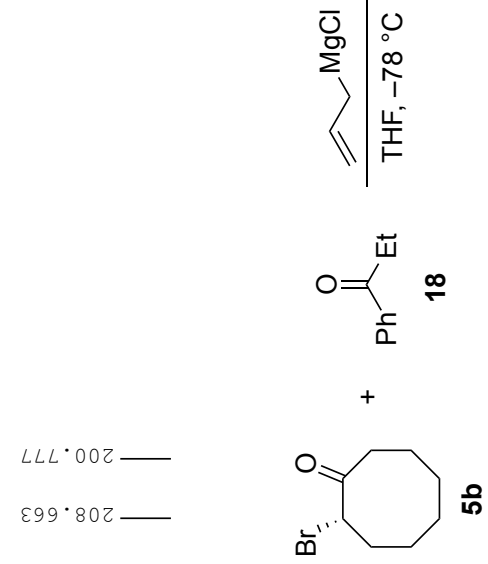

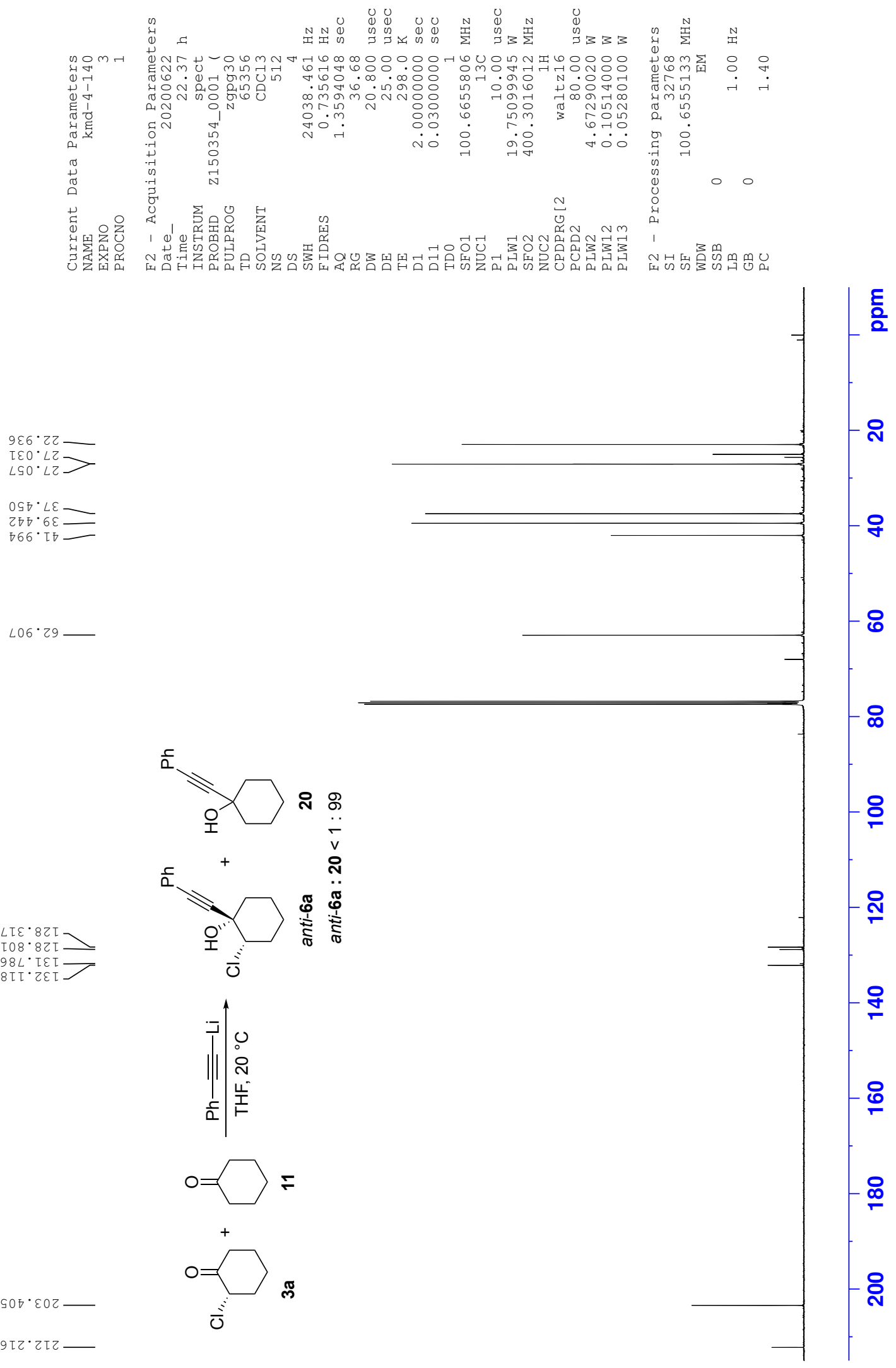

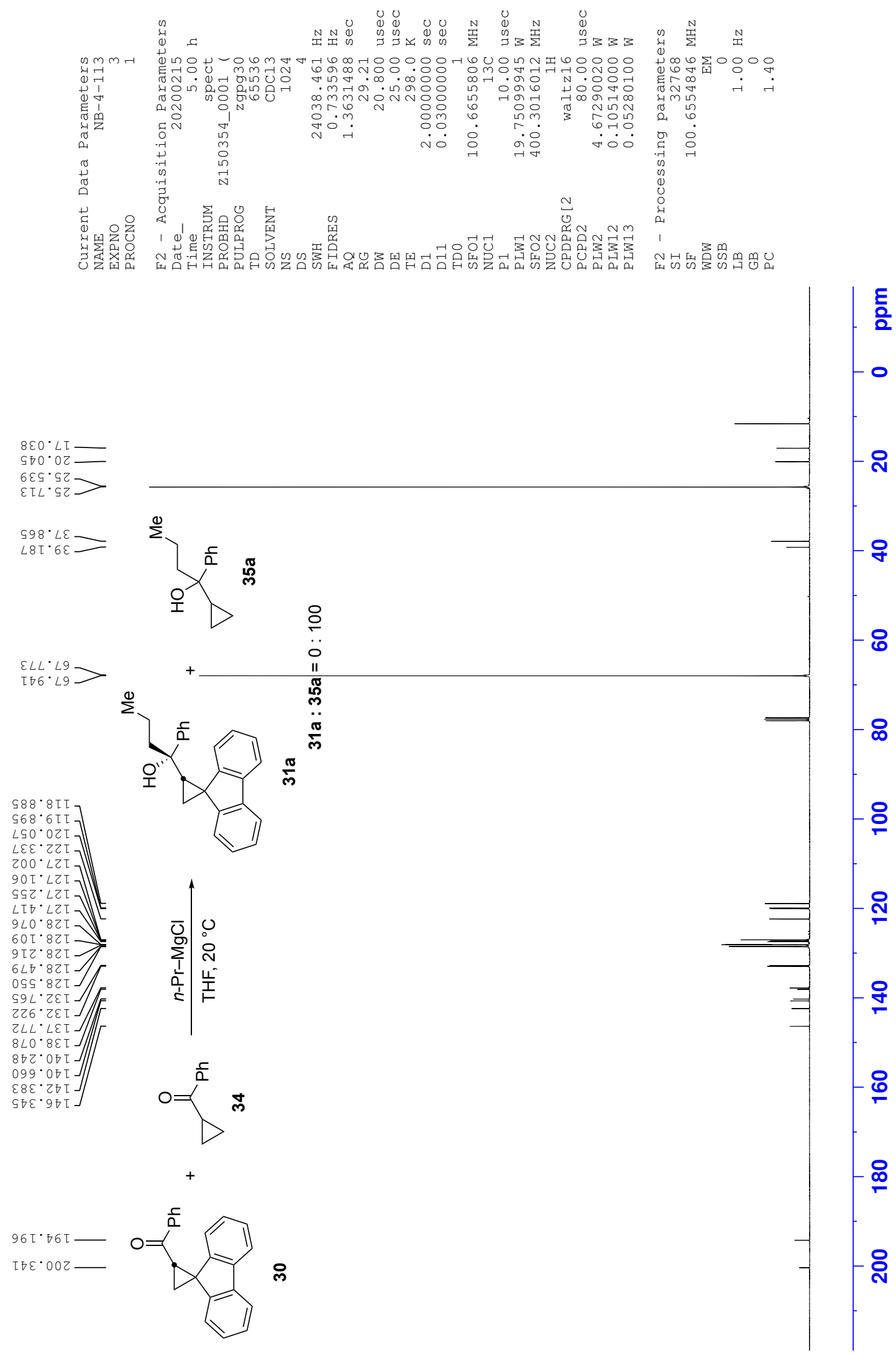

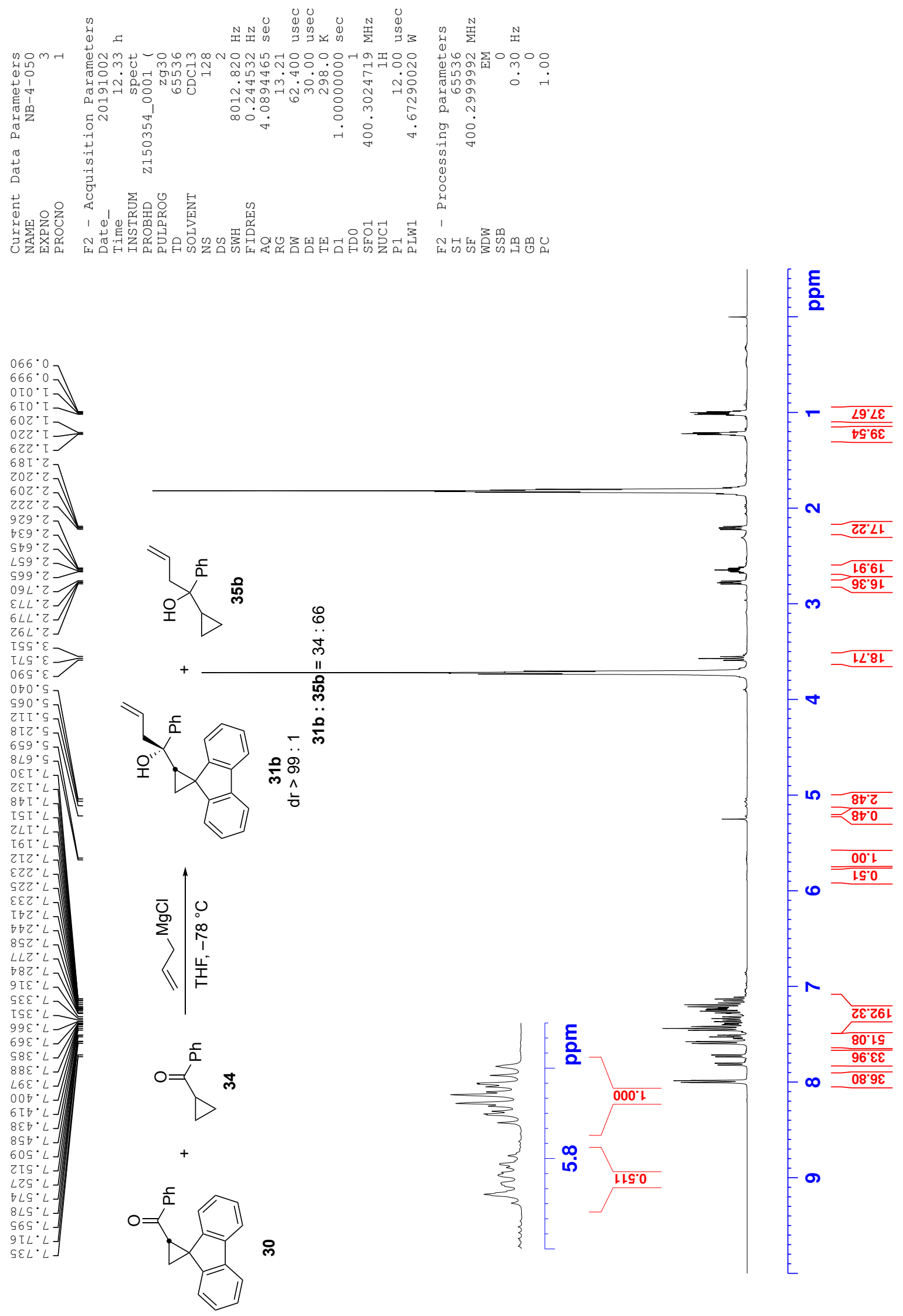

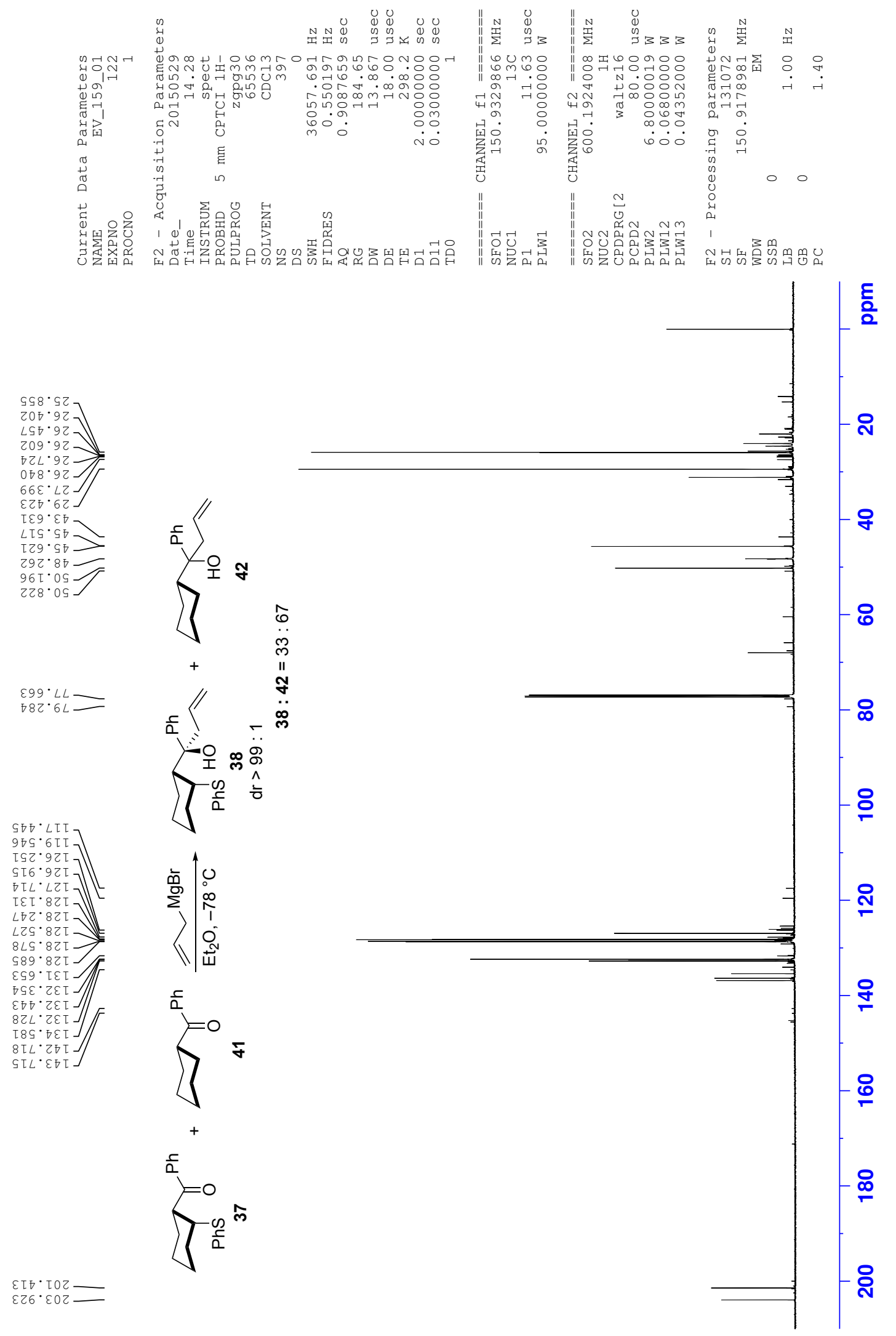

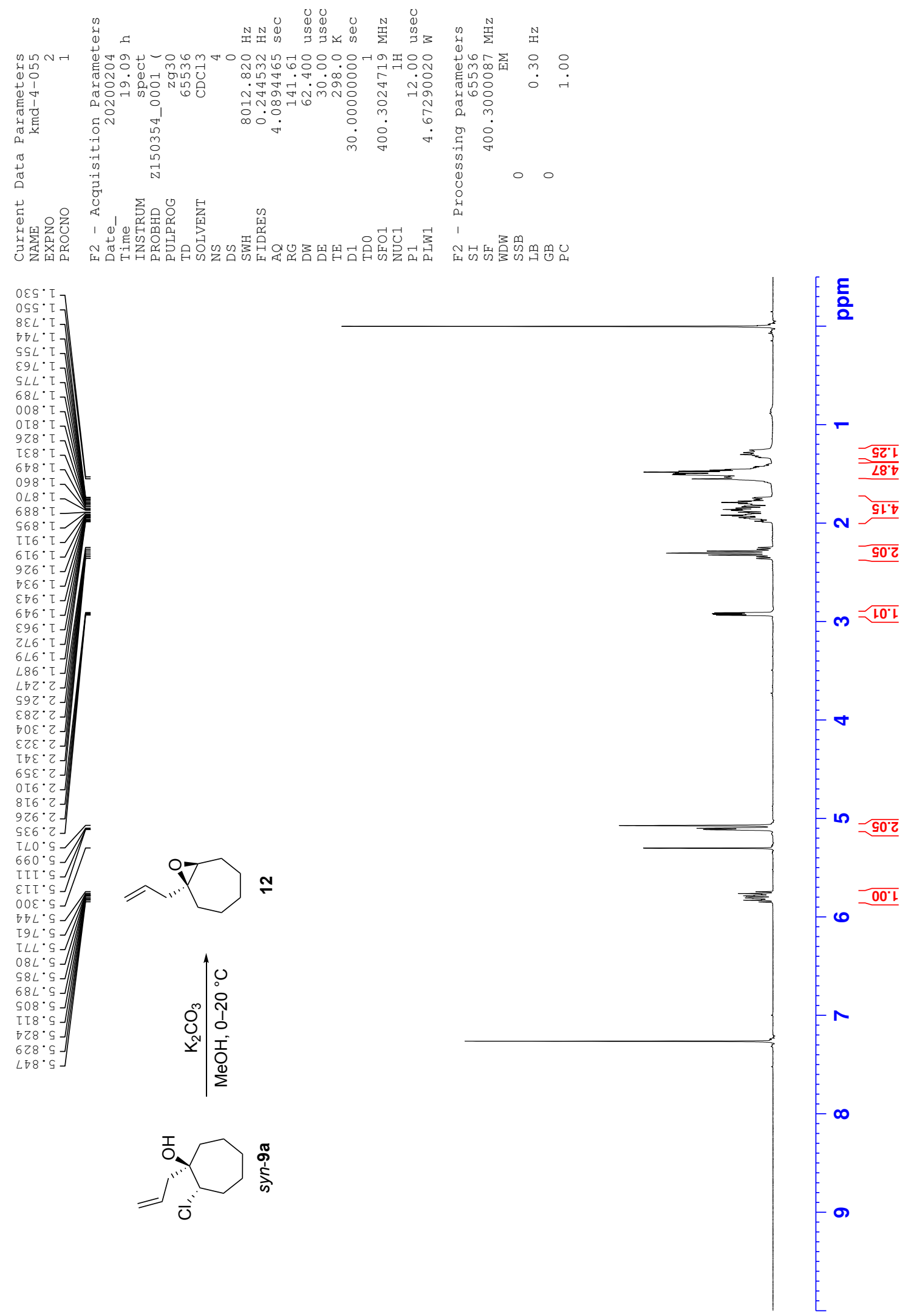

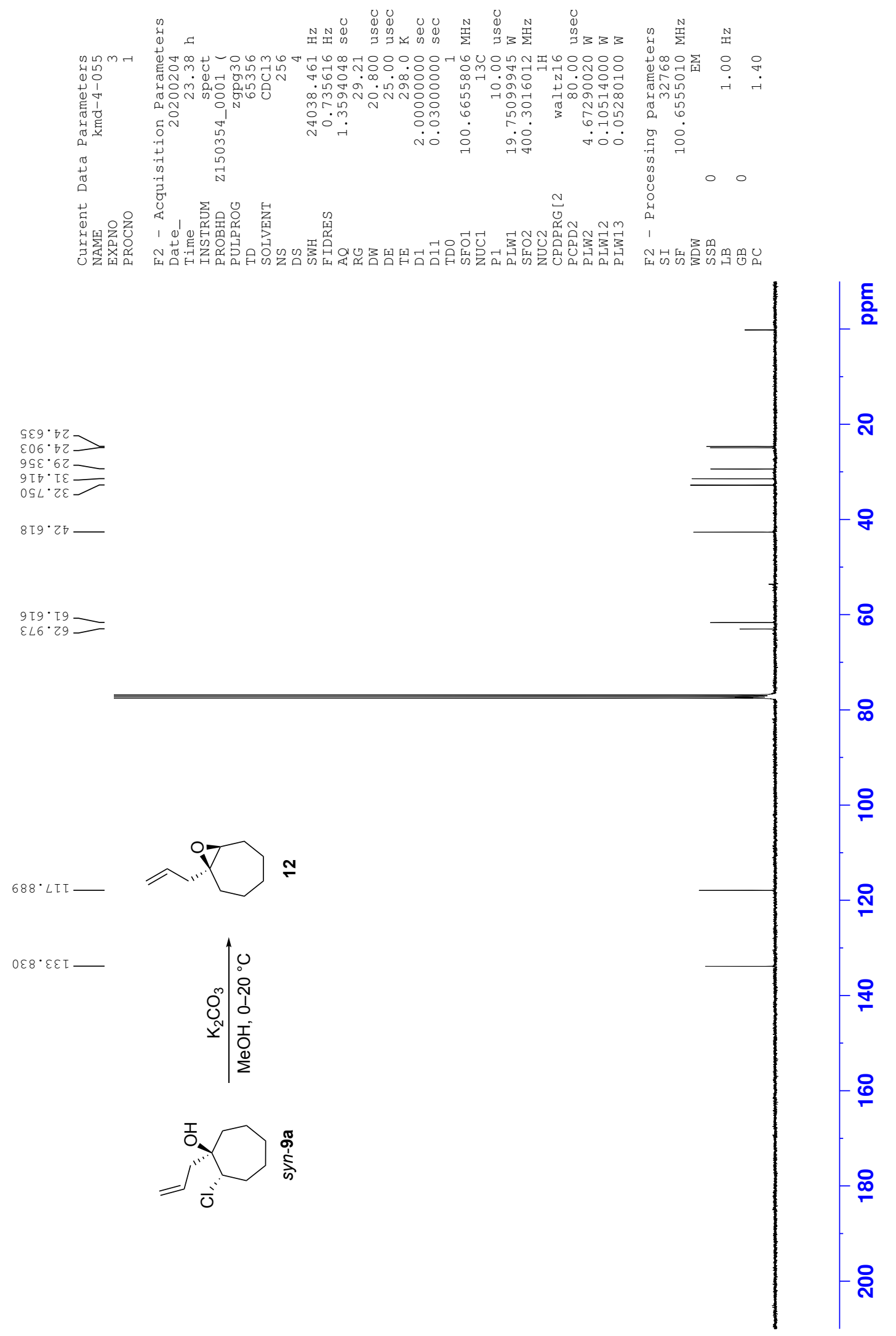

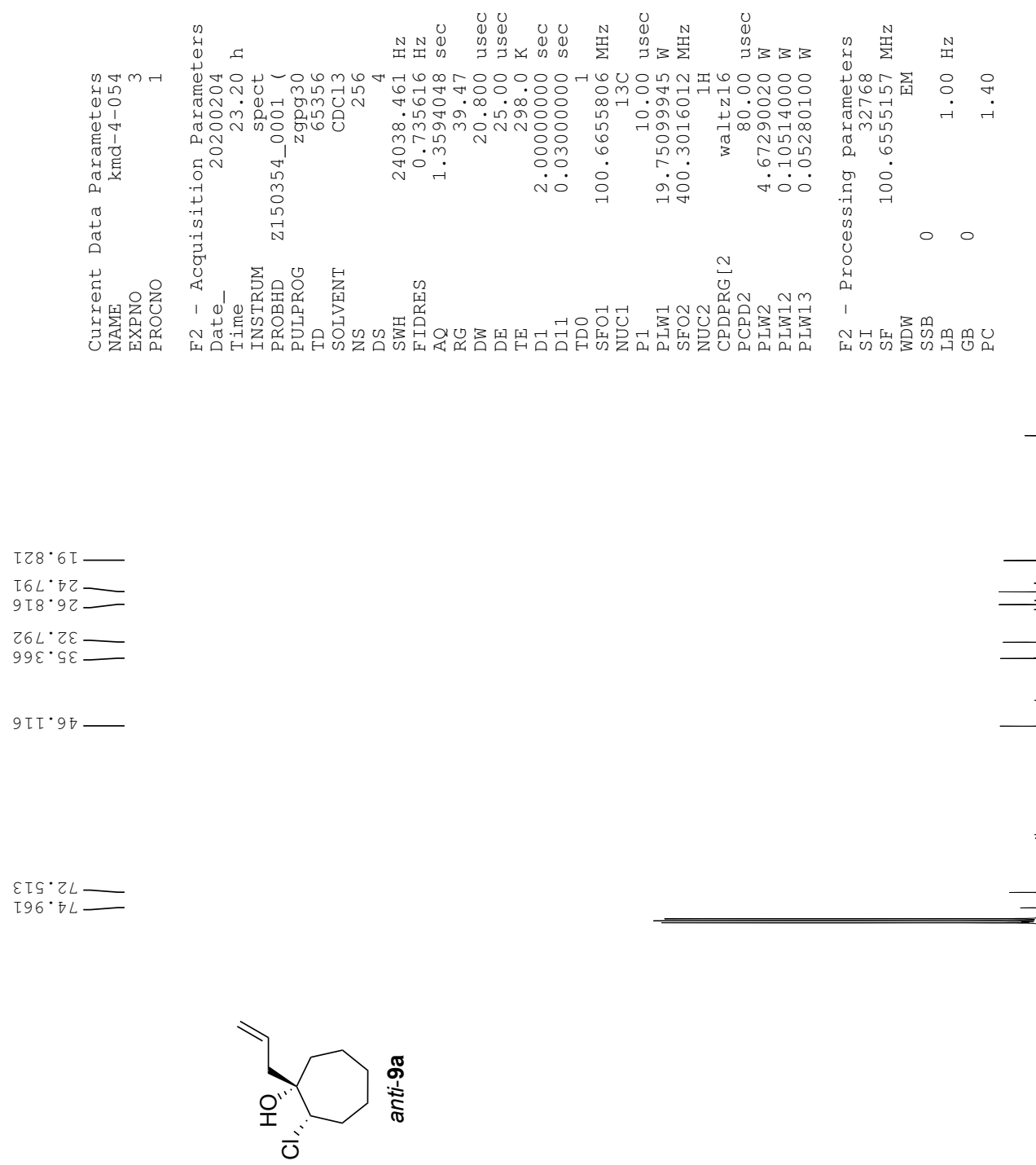

$\varepsilon \sqcap L \cdot 8 I T$

$8 z \varepsilon \cdot \varepsilon \varepsilon \tau$
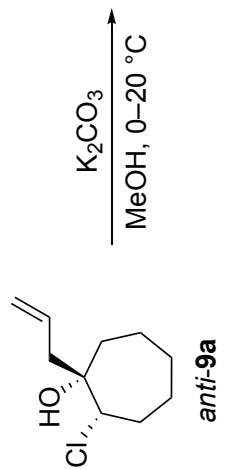

$\stackrel{8}{0}$

$\stackrel{\infty}{\infty}$

ฉั 

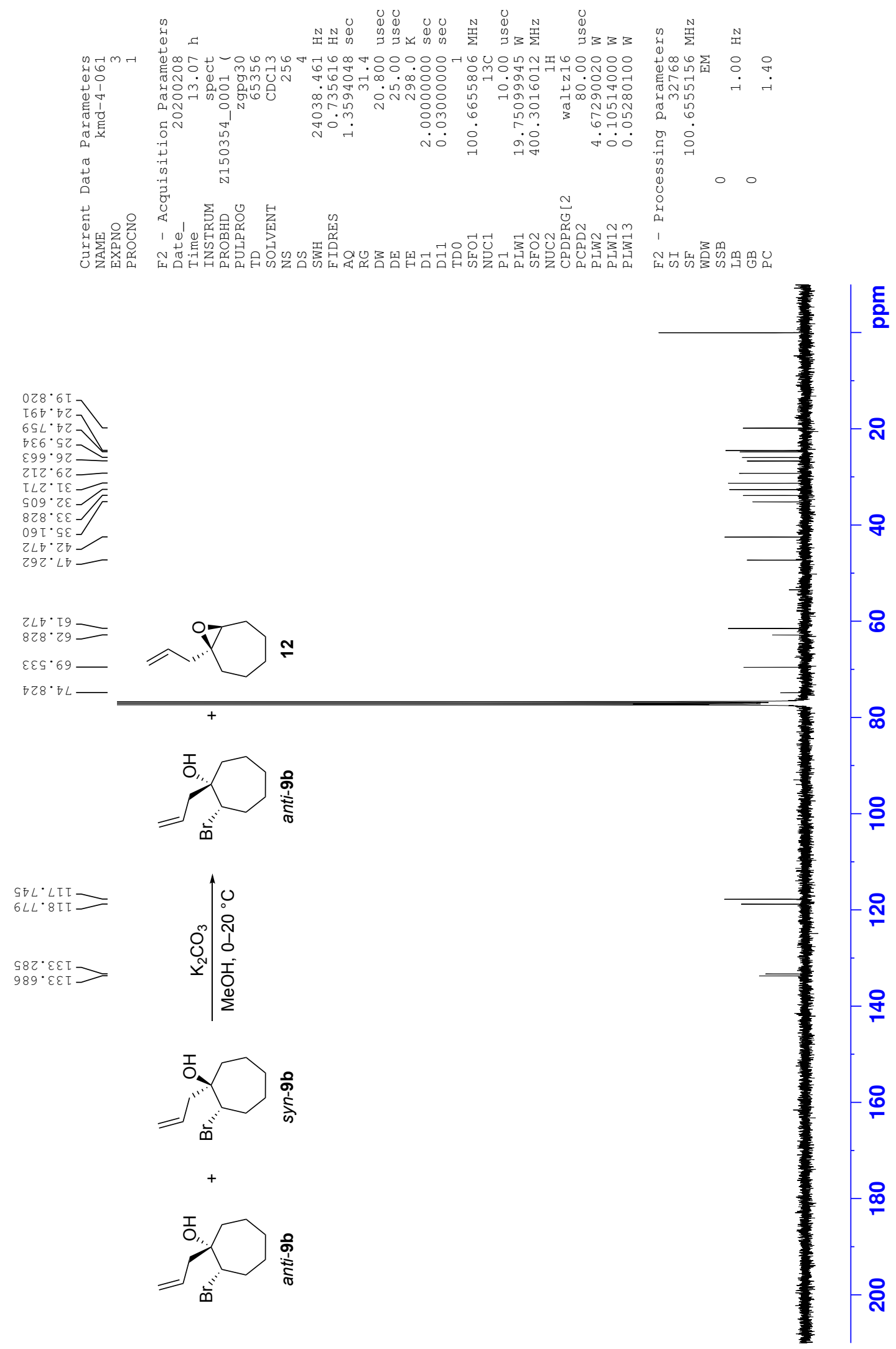

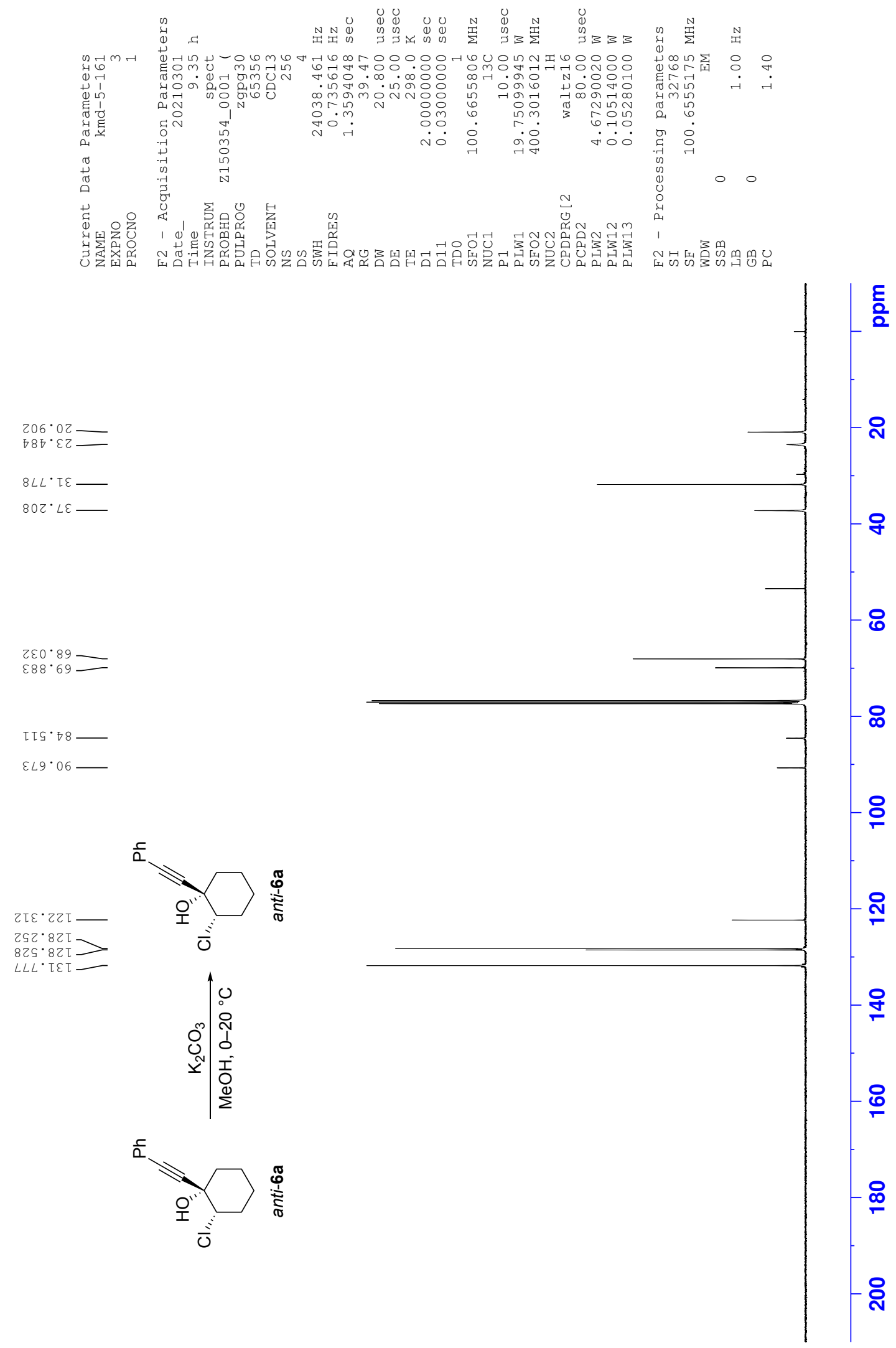

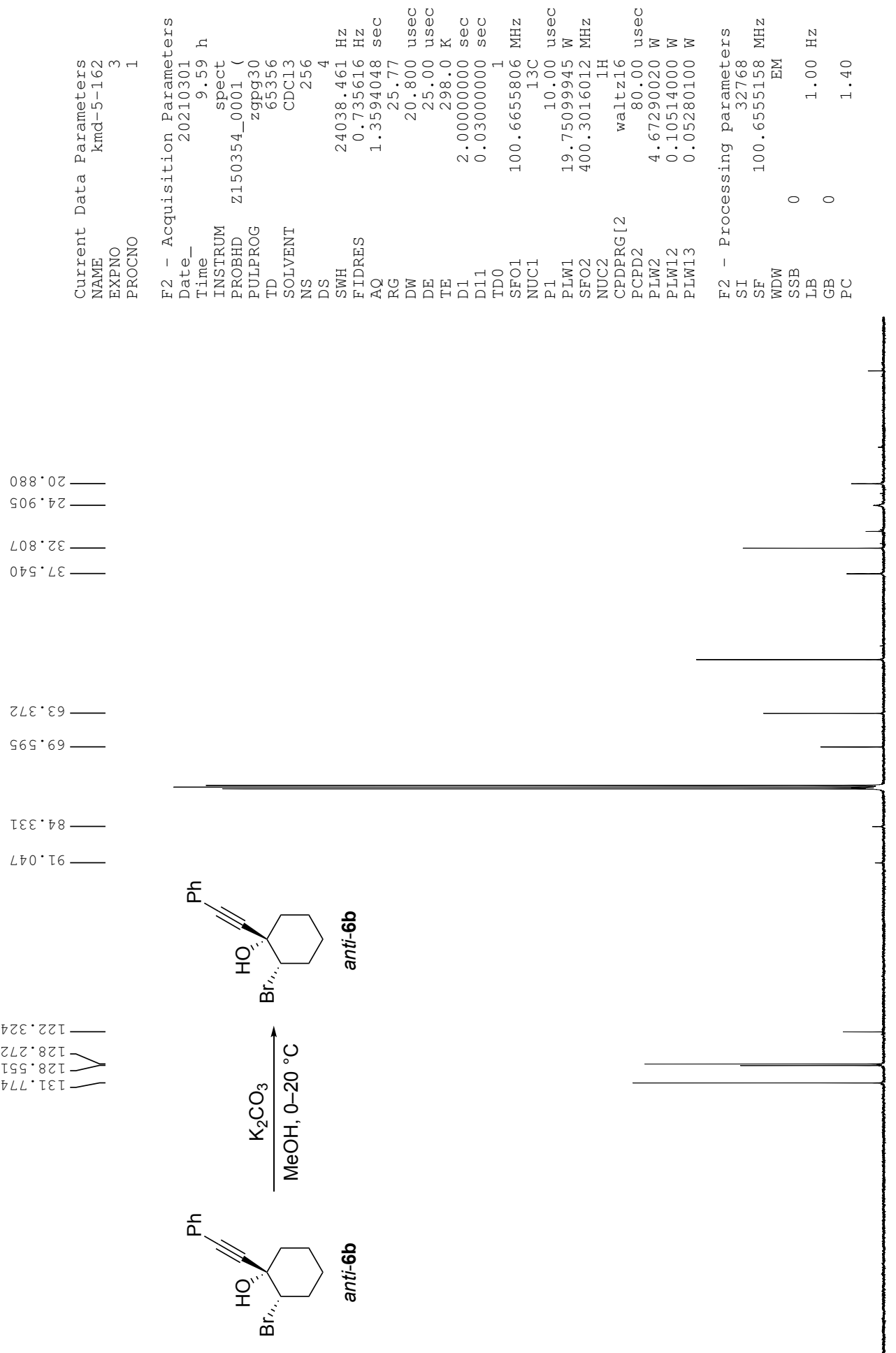

틍

- กิ

q

8

๑

$\angle \mp 0 \cdot 16$

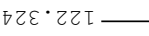

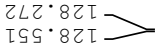

TLL. TEL

$\stackrel{\infty}{\circ}$

$\underline{8}$

ㅇํ 

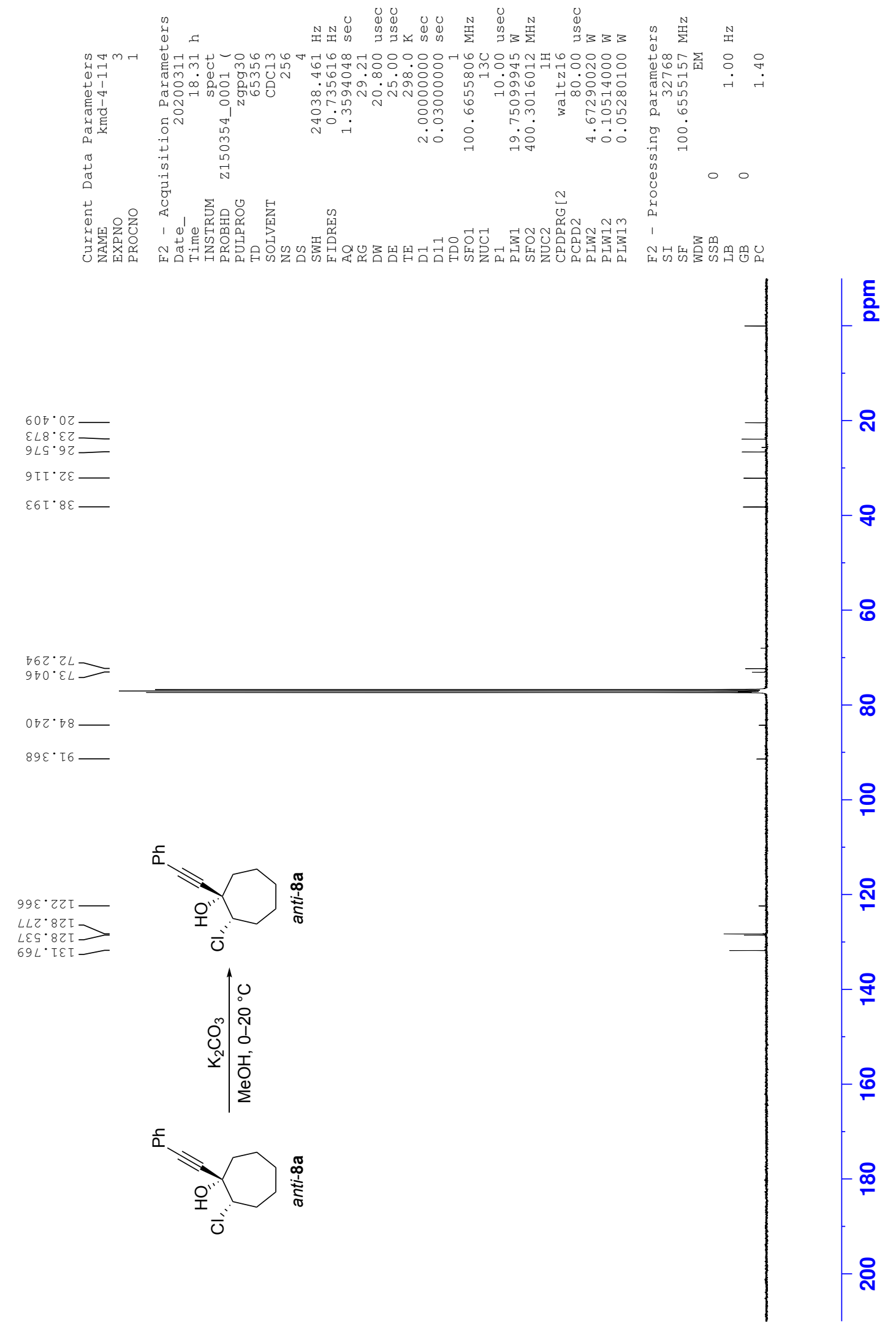

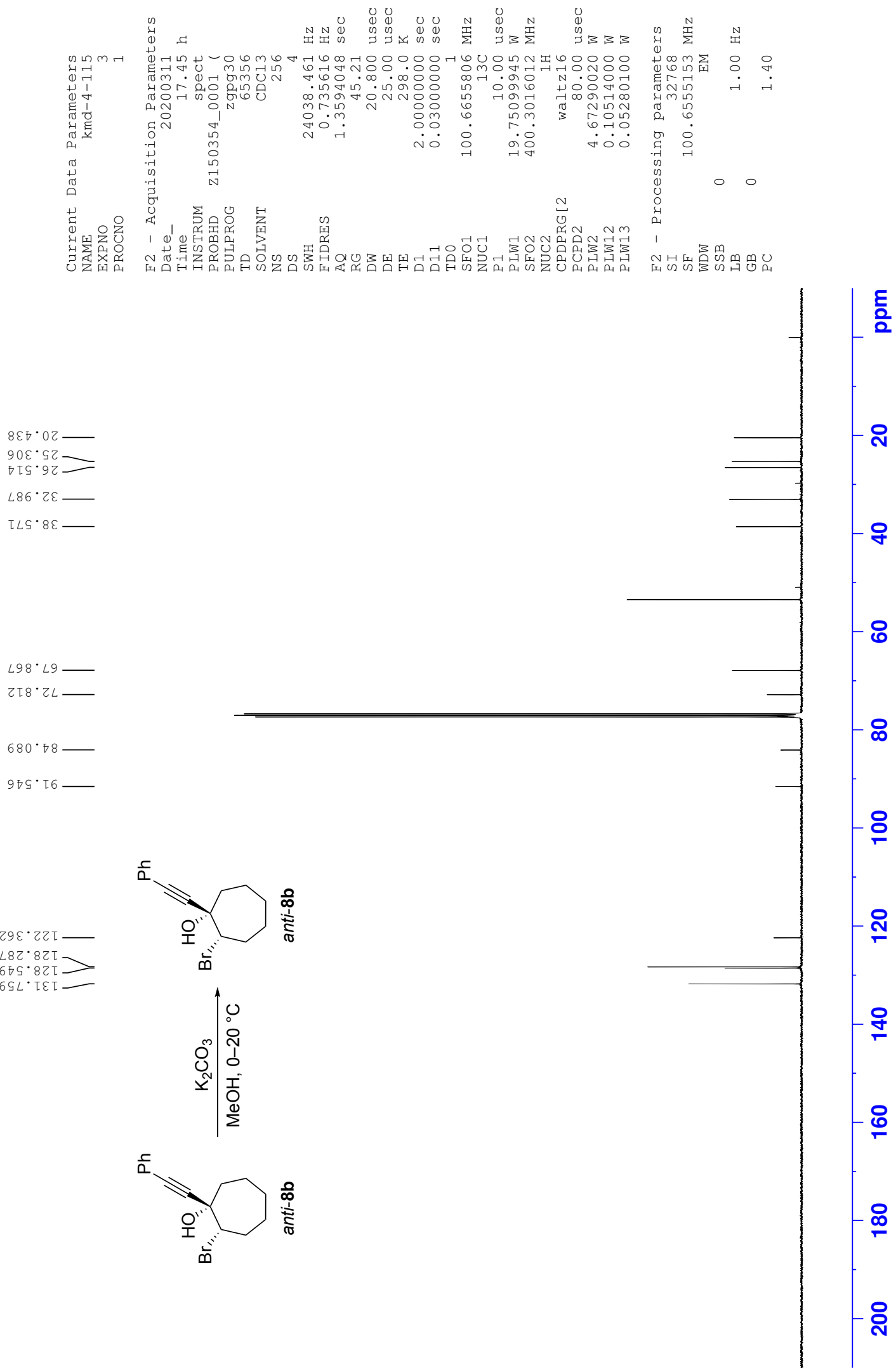